\title{
Cell decompositions and algebraicity of cohomology for quiver Grassmannians
}

\author{
Giovanni Cerulli Irelli ${ }^{a, *}$, Francesco Esposito ${ }^{\mathrm{b}}$, Hans Franzen ${ }^{\mathrm{c}}$, \\ Markus Reineke ${ }^{\mathrm{c}}$ \\ a Sapienza-Università di Roma, Dipartimento S.B.A.I., Via A. Scarpa 10, 00161 \\ Roma, Italy \\ b Università di Padova, Dipartimento di Matematica, Via Trieste 63, 35121 \\ Padova, Italy \\ c Ruhr-University Bochum, Faculty of Mathematics, Universitätsstrasse 150, 44780 \\ Bochum, Germany
}

\section{A R T I C L E I N F O}

Article history:

Received 13 December 2019

Received in revised form 10 August 2020

Accepted 20 November 2020

Available online $\mathrm{xxxx}$

Communicated by Henning Krause

\section{Keywords:}

Quiver Grassmannians

Cellular decomposition

Property (S)

Cluster algebras

\section{A B S T R A C T}

We show that the cohomology ring of a quiver Grassmannian associated with a rigid quiver representation has property (S): there is no odd cohomology and the cycle map is an isomorphism; moreover, its Chow ring admits explicit generators defined over any field. From this we deduce the polynomial point count property. By restricting the quiver to finite or affine type, we are able to show a much stronger assertion: namely, that a quiver Grassmannian associated with an indecomposable (not necessarily rigid) representation admits a cellular decomposition. As a corollary, we establish a cellular decomposition for quiver Grassmannians associated with representations with rigid regular part. Finally, we study the geometry behind the cluster multiplication formula of Caldero and Keller, providing a new proof of a slightly more general result.

(C) 2020 Elsevier Inc. All rights reserved.

\footnotetext{
* Corresponding author.

E-mail addresses: giovanni.cerulliirelli@uniroma1.it (G. Cerulli Irelli), esposito@math.unipd.it (F. Esposito), hans.franzen@rub.de (H. Franzen), Markus.Reineke@ruhr-uni-bochum.de (M. Reineke).
} 


\section{Contents}

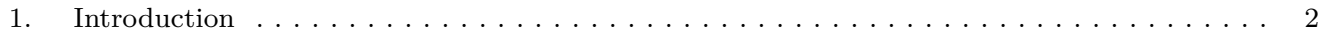

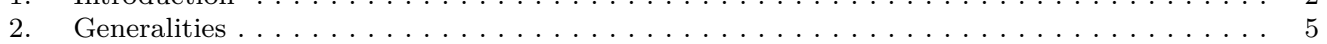

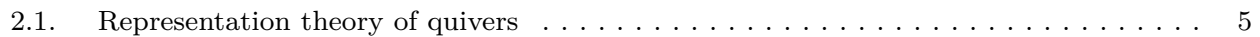

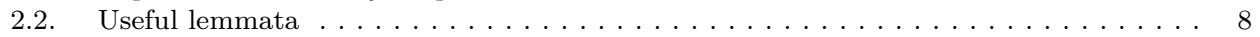

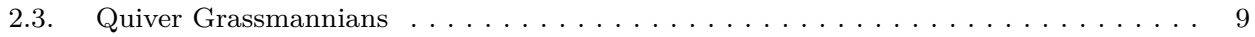

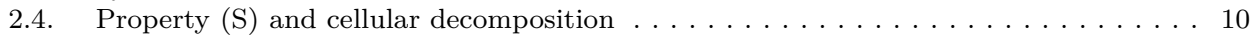

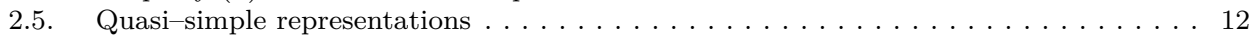

3. Decomposition of quiver Grassmannians induced by short exact sequences . . . . . . . . 13

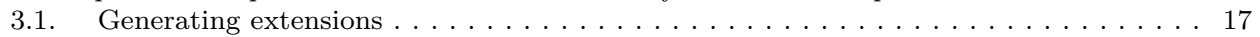

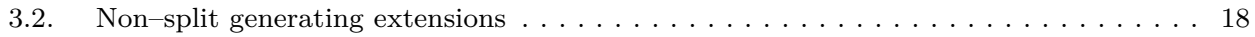

3.3. Description of Ringel reflections in particular cases . . . . . . . . . . . . . . . . . 22

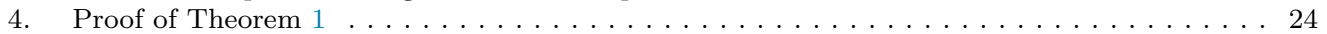

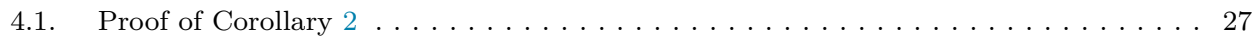

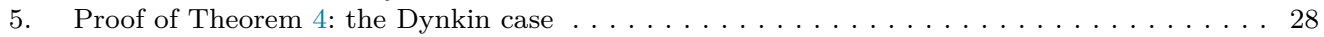

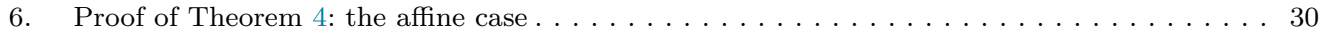

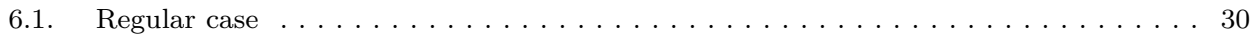

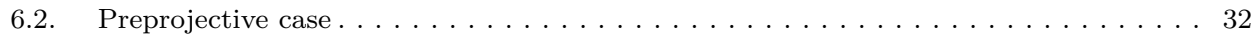

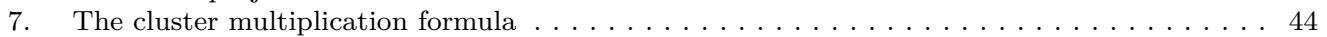

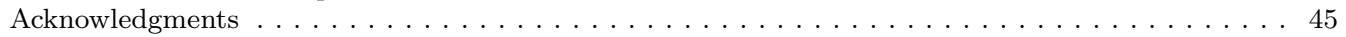

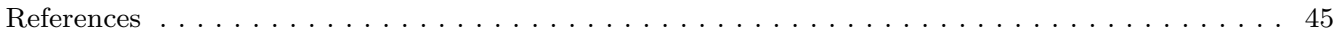

\section{Introduction}

It has long been known that the representation theory of certain algebraic objects (such as Weyl groups, semisimple Lie algebras, Kac-Moody algebras) is intimately linked to the topology of suitable varieties (such as Grassmannians, flag varieties, Schubert varieties, Springer fibers). Relatively more recently, varieties associated with representations of quivers have been used by Lusztig for the study of canonical bases of quantum groups, building on work of Ringel who realized the upper part of quantum groups as Hall algebras of quiver representations over finite fields. In 2001, Fomin and Zelevinsky [26] introduced cluster algebras for the study of dual canonical bases of quantum groups. It was hence expected that there exists a close connection between Hall algebras and cluster algebras. This was realized first by Caldero and Chapoton [5] in 2006 and extended to full generality by Caldero-Keller [7,6], Palu [42], Derksen-Weyman-Zelevinsky [22]: instead of using Hall numbers, Caldero and Chapoton used the Euler characteristic of complex quiver Grassmannians to realize the generators of the cluster algebras. The key of Caldero and Chapoton's realization was a multiplication formula for certain characters of the Grothendieck group of $\operatorname{Rep}_{K}(Q)$ where $Q$ is a Dynkin quiver. This formula is based on the non-trivial fact that quiver Grassmannians for Dynkin quivers have polynomial point count, as a consequence of Ringel's work on Hall numbers. The multiplication formula was then extended by Caldero-Keller [7,6] and Palu [42,43]. A different approach for the proof of a multiplication formula was achieved by Hubery [33] (and independently by Xu [56]) and it is based on Green's theorem and associativity of Hall numbers. 
The positivity conjecture of Fomin-Zelevinsky translates into the positivity conjecture for the Euler-Poincaré characteristic of the quiver Grassmannians associated with rigid representations of acyclic quivers (i.e. quiver representations without self-extensions). This result was proved by Nakajima in [40, Th. A.1] and, independently, by Qin in [44]: They show that such quiver Grassmannians do not have odd cohomology.

Following [17], we say that a smooth projective complex variety $X$ has property $(S)$ if

(1) numerical and rational equivalence on $X$ coincide, in particular the Chow $\operatorname{ring} A^{*}(X)$ is a finitely generated free abelian group,

(2) $H^{2 i+1}(X)=0$ for every $i$, and

(3) the cycle map $A^{i}(X) \rightarrow H^{2 i}(X)$ is an isomorphism for all $i$

(see Section 2.4). For a (finite dimensional) quiver representation $M$ we denote by $\operatorname{Gr}_{\mathbf{e}}(M)$ the projective variety parametrizing subrepresentations of $M$ of dimension vector e, i.e. a quiver Grassmannian attached to $M$. We say that $M$ has property (S) if $\operatorname{Gr}_{\mathbf{e}}(M)$ has property $(\mathrm{S})$ for all e.

Theorem 1. A rigid quiver representation $M$ has property (S).

It is known that the support of a rigid quiver representation is acyclic (see e.g. [49, Prop. 2]) and hence, to prove Theorem 1, it is not restrictive to assume to work with acyclic quivers. The proof of Theorem 1 is given in Section 4 and is based on the Ellingsrud-Strømme decomposition of the diagonal, which provides explicit generators of the Chow rings. Moreover, we prove that quiver Grassmannians attached to rigid quiver representations are irreducible over any field and smooth over $\mathbb{Z}$ (see Proposition 38 and Lemma 45). This has the following consequence that was previously proved by Qin in [44, Th. 3.2.6] employing quantum cluster algebras (see Section 4.1).

Corollary 2. A quiver Grassmannian attached to a rigid representation has polynomial point-count.

Quiver Grassmannians play a central role in cluster theory. In particular, it is important to know the vanishing of odd cohomology (for positivity) and the polynomial count (for quantum cluster variables). These properties were obtained by Nakajima [40] and Qin, [44] with different techniques. We obtain these results by noticing that these varieties admit the decomposition of the diagonal [55].

We recall (see Section 2.4) that a variety $X$ admits a cellular decomposition, or affine paving, if there exists a finite partition $X=X_{1} \amalg \cdots \amalg X_{h}$ such that each piece $X_{i}$ is an affine space, and $X_{1} \cup \cdots \cup X_{i}$ is closed in $X$ for every $i=1, \cdots, h$. Cellular decomposition implies property $(\mathrm{S})$, but in general they are not equivalent. We say that a (finite-dimensional) quiver representation $M$ has property (C) if $\operatorname{Gr}_{\mathbf{e}}(M)$ admits a 
cellular decomposition for all e. Property (C) has been shown to hold for some rigid representations of an oriented tree [35] and for rigid representations of a generalized Kronecker quiver [51].

Conjecture 3. Any rigid quiver representation has property $(C)$.

For quivers of finite or affine type, the following much stronger statement is true and new.

Theorem 4. An indecomposable representation of a quiver of finite or affine type has property $(C)$.

Theorem 4 was proved in [11, Th. 12] for a quiver of type $A$-equioriented, in [10] for the Kronecker quiver, in $[37,36]$ for preprojective representations of type $\tilde{D}_{n}$, it follows from [8] for rigid representations of type $\tilde{A}_{n}$ as a consequence of the existence of a torus action.

The proof of Theorem 4 is based on Theorem 32, which we now illustrate (see Section 3 for details). Let $Q$ be an acyclic quiver (i.e. a quiver without oriented cycles), and let $X$ and $S$ be two $Q$-representations. Any short exact sequence $\xi: 0 \rightarrow X \rightarrow Y \rightarrow S \rightarrow 0$ gives rise to a map $\Psi^{\xi}: \operatorname{Gr}_{\mathbf{e}}(Y) \rightarrow \coprod_{\mathbf{f}+\mathbf{g}=\mathbf{e}} \operatorname{Gr}_{\mathbf{f}}(X) \times \operatorname{Gr}_{\mathbf{g}}(S)$ which restricts to algebraic maps:

$$
\Psi_{\mathbf{f}, \mathbf{g}}^{\xi}: \mathcal{S}_{\mathbf{f}, \mathbf{g}}^{\xi} \rightarrow \operatorname{Gr}_{\mathbf{f}}(X) \times \operatorname{Gr}_{\mathbf{g}}(S) \quad(\text { for every } \mathbf{f}+\mathbf{g}=\mathbf{e})
$$

where $\mathcal{S}_{\mathbf{f}, \mathbf{g}}^{\xi}$ is the preimage of $\operatorname{Gr}_{\mathbf{f}}(X) \times \operatorname{Gr}_{\mathbf{g}}(S)$ under $\Psi^{\xi}$. We prove that if $\xi$ generates $\operatorname{Ext}^{1}(S, X)$, then this is a Zariski-locally trivial affine bundle over its image. So, property (C) of $Y$ can be deduced by the analysis of the images of such maps. If $\operatorname{Ext}^{1}(S, X)=0$, property (C) (resp. property (S)) for $X$ and $S$ implies property (C) (resp. property (S)) for $Y$. So Theorems 1 and 4 imply the following:

Corollary 5. Property $(S)$ holds for a $Q$-representation $M$ with rigid regular part. Moreover, property $(C)$ holds for $M$ if $Q$ is of finite or affine type.

So every representation of a Dynkin quiver has property (C) (see Section 5). We conjecture the same for affine quivers, i.e. that property $(\mathrm{C})$ holds for regular decomposable representations of affine quivers. For a wild quiver, property $(\mathrm{C})$ cannot hold for every representation. Indeed Ringel has recently shown [50] the following striking result: given any wild quiver $Q$ and any projective variety $X$ there exists a representation $N$ of $Q$ and a dimension vector e such that $X \cong \mathrm{Gr}_{\mathbf{e}}(N)$.

If $\xi$ generates $\operatorname{Ext}^{1}(S, X) \neq 0$, the maps $\Psi_{\mathbf{f}, \mathbf{g}}^{\xi}$ are not surjective and we determine their images. We prove that the following are well-defined (see Lemma 27):

$$
X_{S}:=\max \left\{N \subseteq_{Q} X \mid[S, X / N]^{1}=1\right\}, \quad S^{X}:=\min \left\{N \subseteq_{Q} S \mid[N, X]^{1}=1\right\},
$$


where we use the standard shorthand notation $[A, B]^{1}:=\operatorname{dim}_{\operatorname{Ext}^{1}}(A, B)$. It turns out that the image of $\Psi_{\mathbf{f}, \mathbf{g}}^{\xi}$ is the complement inside $\operatorname{Gr}_{\mathbf{f}}(X) \times \operatorname{Gr}_{\mathbf{g}}(S)$ of the closed subset $\left(\operatorname{Gr}_{\mathbf{f}}\left(X_{S}\right) \times \operatorname{Gr}_{\mathbf{g}-\operatorname{dim} S^{X}}\left(S / S^{X}\right)\right)$. From this description, we deduce a multiplication formula for the cluster character with coefficients $C C: \operatorname{Rep}_{K}(Q) \rightarrow$ $\mathbb{Z}\left[y_{1}, \cdots, y_{n}, x_{1}^{ \pm 1}, \cdots, x_{n}^{ \pm 1}\right]$ (see Section 7 for details).

Theorem 6. Under the previous hypothesis $C C(X) C C(S)=C C(Y)+\mathbf{y}^{\operatorname{dim} S^{X}} C C\left(X_{S} \oplus\right.$ $\left.S / S^{X}\right) \mathbf{x}^{\mathbf{f}}$.

Multiplication formulas for cluster characters were studied intensively by several authors $[7,6,42,43,33,56]$ in much more general contexts. In $[7,43,33,56]$ the assumption $[S, X]^{1}=1$ is dropped, and it would be interesting to know if there exists a more general reduction theorem, underlying those results.

\section{Generalities}

\subsection{Representation theory of quivers}

In this section we collect some well-known facts about quiver representations. Standard references are [14], [15], [3], [48], [2]. Let $Q=\left(Q_{0}, Q_{1}, s, t\right)$ be a finite connected quiver with set of vertices $Q_{0}$ of cardinality $n$, finite set of arrows $Q_{1}$ and every arrow $\alpha \in Q_{1}$ is oriented from its starting vertex $s(\alpha) \in Q_{0}$ towards its terminal vertex $t(\alpha) \in Q_{0}$ and we write $\alpha: s(\alpha) \rightarrow t(\alpha)$. Given a field $K$, we consider the category $\operatorname{Rep}_{K}(Q)$ of finite-dimensional $Q$-representations over $K$. For a representation $M$ of $Q$ we denote by $M_{i}$ the $K$-vector space attached to vertex $i \in Q_{0}$, and by $M_{\alpha}: M_{s(\alpha)} \rightarrow M_{t(\alpha)}$ the $\mathrm{K}$-linear map attached to an arrow $\alpha \in Q_{1}$. The category $\operatorname{Rep}_{K}(Q)$ of finitedimensional $Q$-representations is equivalent to the category $A-\bmod$ of finite dimensional modules over the path algebra $A=K Q$ of $Q$. We work with left modules and we do not distinguish between objects of $\operatorname{Rep}_{K}(Q)$ and $A-\bmod$. The category $\operatorname{Rep}_{K}(Q)$ is abelian, Krull-Schmidt and hereditary, i.e. every module has projective dimension at most one, i.e. the functors $\operatorname{Ext}_{Q}^{i}(-,-)$ vanish for $i \geq 2$. Given two $Q$-representations, we often use the standard notation:

$$
[M, N]:=\operatorname{dim}_{K} \operatorname{Hom}_{Q}(M, N), \quad[M, N]^{1}:=\operatorname{dim}_{K} \operatorname{Ext}_{Q}^{1}(M, N)
$$

When $Q$ is acyclic, the simple objects of $\operatorname{Rep}_{K}(Q)$ are one-dimensional and supported on a single vertex. Given a vertex $k \in Q_{0}$ we denote by $S_{k}$ the corresponding simple, by $P_{k}$ its projective cover and by $I_{k}$ its injective envelope. The Nakayama functor $\nu:=$ $D \operatorname{Hom}(-, A)$ establishes an equivalence $\nu: \operatorname{Proj}(A) \rightarrow \operatorname{Inj}(A)$ from the category of projectives to the category of injectives, and it is characterized by $\nu\left(P_{k}\right)=I_{k}$.

\subsubsection{Auslander-Reiten theory of $\operatorname{Rep}_{K}(Q)$}

The category $\operatorname{Rep}_{K}(Q)$ is endowed with the two endofunctors $\tau:=D \operatorname{Ext}^{1}(-, A)$ and $\tau^{-}:=\operatorname{Ext}^{1}(D(-), A)$, the Auslander-Reiten translate and its quasi-inverse $(D=$ 
$\operatorname{Hom}_{K}(-, K)$ denotes the standard duality). Notice that $\tau$ is left-exact and $\tau^{-}$is rightexact. For any two $A$-modules $L$ and $M$ there are functorial isomorphisms called Auslander-Reiten formulas:

$$
\operatorname{Ext}^{1}(L, M) \cong D \operatorname{Hom}(M, \tau L) \cong D \operatorname{Hom}\left(\tau^{-} M, L\right)
$$

Given an indecomposable non-projective $Q$-representation $M$ there exists a short exact sequence

$$
0 \rightarrow \tau M \rightarrow E \rightarrow M \rightarrow 0
$$

which is almost split, i.e. it is non-split, every morphism $\tau M \rightarrow Z$ which is not split epi factors through $E$ and every morphism $Z \rightarrow M$ which is not split mono factors through $E$.

An indecomposable module $M$ is called preprojective (resp. preinjective) if there exist $k \geq 0$ and $\ell \in Q_{0}$ such that $M \cong \tau^{-k} P_{\ell}$ (resp. $M \cong \tau^{k} I_{\ell}$ ) and regular if $\tau^{ \pm k} M$ is non-zero for all $k$. We say that $M$ is preprojective (resp. preinjective, regular) if all its indecomposable direct summands are preprojective (resp. preinjective, regular). We denote respectively by $\mathcal{P}, \mathcal{R}, \mathcal{I}$ the full subcategory of $A$-mod whose objects are preprojectives, regular, preinjectives, respectively. The category $\mathcal{P}$ is closed under taking submodules; the category $\mathcal{I}$ is closed under taking quotients. Moreover,

$$
\operatorname{Hom}(\mathcal{I}, \mathcal{R})=\operatorname{Hom}(\mathcal{I}, \mathcal{P})=\operatorname{Hom}(\mathcal{R}, \mathcal{P})=0=\operatorname{Ext}^{1}(\mathcal{R}, \mathcal{I})=\operatorname{Ext}^{1}(\mathcal{P}, \mathcal{I})=\operatorname{Ext}^{1}(\mathcal{P}, \mathcal{R})
$$

Every module $M$ admits a unique split filtration $M^{\prime} \subseteq M^{\prime \prime} \subseteq M$ where $M^{\prime} \in \mathcal{I}$, $M^{\prime \prime} / M^{\prime} \in \mathcal{R}$ and $M / M^{\prime \prime} \in \mathcal{P}$; these are called the preinjective, regular and preprojective parts of $M$, respectively.

Given two indecomposable $Q$-representations $X$ and $Y$, let $\operatorname{rad}(X, Y) \subseteq \operatorname{Hom}_{Q}(X, Y)$ be the vector subspace of non-invertible morphisms from $X$ to $Y$. Inside $\operatorname{rad}(X, Y)$ there is the subspace $\operatorname{rad}^{2}(X, Y)$ of morphisms $f=f_{2} \circ f_{1}$ such that $f_{1} \in \operatorname{rad}(X, M)$ and $f_{2} \in \operatorname{rad}(M, Y)$ for some $M$. The quotient space is denoted by $\operatorname{Irr}(X, Y)=$ $\operatorname{rad}(X, Y) / \operatorname{rad}^{2}(X, Y)$. A morphism $f \in \operatorname{Hom}_{Q}(X, Y)$ is called irreducible if $f \in$ $\operatorname{rad}(X, Y) \backslash \operatorname{rad}^{2}(X, Y)$. The Auslander-Reiten quiver $\Gamma(Q)$ of $Q$ is the quiver whose vertices are isoclasses of indecomposable $Q$-representations and the number of arrows between two vertices $[X]$ and $[Y]$ (corresponding to the two indecomposables $X$ and $Y$ ) equals $\operatorname{dim}_{K} \operatorname{Irr}(X, Y)$. Many properties of $\operatorname{Rep}_{K}(Q)$ can be read from $\Gamma(Q)$. The quiver $\Gamma(Q)$ is finite if and only if $Q$ is Dynkin. If $Q$ is not Dynkin, $\Gamma(Q)=\Gamma(\mathcal{P}) \cup \Gamma(\mathcal{R}) \cup \Gamma(\mathcal{I})$ where $\Gamma(\mathcal{P})(\operatorname{resp} . \Gamma(\mathcal{R})$, resp. $\Gamma(\mathcal{I}))$ is the Auslander-Reiten quiver of $\mathcal{P}$ (resp. $\mathcal{R}$, resp. $\mathcal{I}$ ). Notice that $\Gamma(\mathcal{P})$ (resp. $\Gamma(\mathcal{I})$ ) contains all the indecomposable projectives (resp. injectives), and can be described combinatorially via the knitting algorithm. The regular components are described by Ringel [46].

Given two indecomposable representations $X$ and $Y$, a sectional morphism $f: X \rightarrow Y$ is a composition 


$$
f: X \stackrel{f_{1}}{\longrightarrow} X_{1} \stackrel{f_{2}}{\longrightarrow} \cdots \stackrel{f_{t-1}}{\longrightarrow} X_{t-1} \stackrel{f_{t}}{\longrightarrow} X_{t}=Y
$$

of irreducible morphisms $f_{i}: X_{i-1} \rightarrow X_{i}$ such that $X_{i} ¥ \tau X_{i+2}$. Every sectional morphism is represented by an oriented (connected) path between $[X]$ and $[Y]$ in $\Gamma(Q)$ which does not contain a path of the form $[\tau M] \rightarrow[Z] \rightarrow[M]$.

\subsubsection{Euler form and representation type}

Given a representation $M$ of $Q$ we denote by $\operatorname{dim} M:=\left(\operatorname{dim}_{K}\left(M_{i}\right)\right) \in \mathbb{Z}_{\geq 0}^{Q_{0}}$ the dimension vector of $M$ and we sometimes use the shorthand notation $\mathbf{d}^{M}=\left(d_{i}^{M}\right)$. Given two $Q$-representations $M$ and $N$, Ringel defined the following map

$$
\Phi_{M}^{N}: \bigoplus_{i \in Q_{0}} \operatorname{Hom}_{K}\left(M_{i}, N_{i}\right) \rightarrow \bigoplus_{\alpha \in Q_{1}} \operatorname{Hom}_{K}\left(M_{s(\alpha)}, N_{t(\alpha)}\right):\left(f_{i}\right) \mapsto\left(N_{\alpha} \circ f_{s(\alpha)}-f_{t(\alpha)} \circ M_{\alpha}\right)
$$

One sees that $\operatorname{Ker}\left(\Phi_{M}^{N}\right)=\operatorname{Hom}_{Q}(M, N)$ and $\operatorname{Coker}\left(\Phi_{M}^{N}\right) \cong \operatorname{Ext}_{Q}^{1}(M, N)$. In particular we get

$$
[M, N]-[M, N]^{1}=\langle\operatorname{dim} M, \operatorname{dim} N\rangle:=\sum_{i \in Q_{0}} d_{i}^{M} d_{i}^{N}-\sum_{\alpha \in Q_{1}} d_{s(\alpha)}^{M} d_{t(\alpha)}^{N}
$$

The bilinear form $\langle-,-\rangle: \mathbb{Z}^{Q_{0}} \times \mathbb{Z}^{Q_{0}} \rightarrow \mathbb{Z}$ is called the Euler-Ringel form of the quiver $Q$. The corresponding symmetric form $b_{Q}(\mathbf{x}, \mathbf{y}):=\frac{1}{2}(\langle\mathbf{x}, \mathbf{y}\rangle+\langle\mathbf{y}, \mathbf{x}\rangle)$ defines a quadratic form $q_{Q}(\mathbf{x})=b_{Q}(\mathbf{x}, \mathbf{x})$ which plays an important rôle for the representation theory of $Q$. Indeed $q_{Q}$ is positive definite if and only if $Q$ is an orientation of a simply-laced Dynkin diagram of type $A_{n}(n \geq 1), D_{n}(n \geq 4), E_{6}, E_{7}$ and $E_{8}$. The quiver $Q$ is called affine if $q_{Q}$ is positive semi-definite, but not definite. This happens if and only if $Q$ is an orientation of a simply-laced extended Dynkin diagram of type $\tilde{A}_{n}(n \geq 1), \tilde{D}_{n}$ $(n \geq 4), \tilde{E}_{6}, \tilde{E}_{7}$ and $\tilde{E}_{8}$ (see Table 1 ). In this case the kernel of $q_{Q}$ is generated by the minimal positive imaginary root that is denoted with $\delta$. Notice that $\delta$ does not depend on the orientation of the quiver, but only on its underlying graph. In Table 1 we recollect the minimal positive imaginary root in each type. The quiver $Q$ is called wild if $q_{Q}$ is indefinite.

By Gabriel's theorem, $Q$ admits a finite number of indecomposable representations (up to isomorphism) precisely when $q_{Q}$ is positive definite. In this case the dimension vectors of the indecomposables are precisely $\mathbf{x} \in \mathbb{Z}_{\geq 0}^{Q_{0}}$ such that $q_{Q}(\mathbf{x})=1$. These dimension vectors are precisely the positive roots of the semisimple complex Lie algebra corresponding to $Q$.

If $Q$ is affine, the dimension vectors of the indecomposable $Q$-representations are precisely those $\mathbf{x} \in \mathbb{Z}_{\geq 0}^{Q_{0}}$ such that $q_{Q}(\mathbf{x}) \leq 1$. Moreover $q_{Q}(\mathbf{x})=0$ if and only if $\mathbf{x}$ is a multiple of $\delta$. In section 6 , we will give more information concerning the representation theory of affine quivers. 


\subsubsection{Representation varieties and group actions}

A $Q$-representation $M$ is called rigid if $[M, M]^{1}=0$. It is called exceptional, if it is indecomposable and rigid. Finally, $M$ is called a brick if $[M, M]=1$. Given a dimension vector $\mathbf{d} \in \mathbb{Z}_{\geq 0}^{Q_{0}}$, the vector space $R_{\mathbf{d}}(Q):=\bigoplus_{\alpha \in Q_{1}} \operatorname{Hom}_{K}\left(K^{d_{s(\alpha)}}, K^{d_{t(\alpha)}}\right)$ parametrizes the $Q$-representations of dimension vector $\mathbf{d}$ and we identify points in $R_{\mathbf{d}}$ with representations. This vector space is acted upon by the group $G_{\mathbf{d}}:=\prod_{i \in Q_{0}} G L_{d_{i}}(K)$ via base change. The $G_{\mathbf{d}}$-orbits are in bijection with isoclasses of $Q$-representations of dimension vector $\mathbf{d}$. Given a point $M \in R_{\mathbf{d}}(Q)$, the corresponding orbit has codimension $[M, M]^{1}$ in $\mathrm{R}_{\mathbf{d}}(Q)$. In particular, a rigid representation has dense orbit and hence for any dimension vector $\mathbf{d}$ there exists at most one rigid representation (up to isomorphism) that we denote by $M_{\mathbf{d}}$.

\subsection{Useful lemmata}

We recall two lemmata which are known to experts. We include the proofs for convenience of the reader.

Lemma 7 (Happel-Ringel). [32, Lem. 4.1] Let $X$ and $Y$ be indecomposable $Q$-representations. If $[Y, X]^{1}=0$, then a non-zero map $f: X \rightarrow Y$ is either injective or surjective.

Proof. We consider the two short exact sequences

$$
\xi_{1}: 0 \longrightarrow \operatorname{ker} f \longrightarrow X \longrightarrow \operatorname{im} f \longrightarrow 0, \quad \xi_{2}: 0 \longrightarrow \operatorname{im} f \stackrel{\iota}{\longrightarrow} Y \longrightarrow \operatorname{coker} f \longrightarrow 0 .
$$

Apply $\operatorname{Hom}(-, \operatorname{ker} f)$ to $\xi_{2}$ and get a surjective morphism

$$
\operatorname{Ext}^{1}(Y, \operatorname{ker} f) \longrightarrow \operatorname{Ext}^{1}(\operatorname{im} f, \operatorname{ker} f)
$$

which implies the existence of a commutative diagram with exact rows

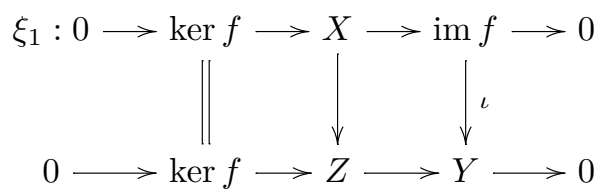

and hence of a short exact sequence

$$
0 \longrightarrow X \longrightarrow Z \oplus \operatorname{im} f \longrightarrow Y \longrightarrow 0 \text {. }
$$

Since $\operatorname{Ext}^{1}(Y, X)=0$, by the Krull-Schmidt property, $\operatorname{im} f=Y$ or $\operatorname{im} f \cong X$.

The following lemma is attributed to Unger, but we could not find a published version of it. 
Lemma 8 (Unger). Let $X$ and $Y$ be exceptional representations of $Q$ such that $[Y, X]^{1}=$ 0. Let $f: X \hookrightarrow Y$ be a monomorphism between them and let $S$ be its cokernel. Then $[S, S]=1$. In particular $S$ is indecomposable. Moreover

$$
[S, S]^{1}=[X, Y]-1
$$

Proof. Since $Y$ is exceptional and $S$ is a quotient of $Y$ we have $[Y, S]^{1} \leq[Y, Y]^{1}=0$. We consider the short exact sequence $\xi: 0 \rightarrow X \stackrel{f}{\rightarrow} Y \rightarrow S \rightarrow 0$. We apply the functor $\operatorname{Hom}(Y,-)$ to $\xi$ and get:

$$
0 \longrightarrow \operatorname{Hom}(Y, X) \longrightarrow \operatorname{Hom}(Y, Y) \longrightarrow \operatorname{Hom}(Y, S) \longrightarrow \operatorname{Ext}^{1}(Y, X)=0
$$

from which we deduce that $[Y, S] \leq[Y, Y]=1$ and hence $[Y, S]=1$ because $S$ is a non-zero quotient of $Y$. We then apply the functor $\operatorname{Hom}(-, S)$ and get

$0 \longrightarrow \operatorname{Hom}(S, S) \longrightarrow \operatorname{Hom}(Y, S) \stackrel{F}{\longrightarrow} \operatorname{Hom}(X, S) \longrightarrow \operatorname{Ext}^{1}(S, S) \longrightarrow \operatorname{Ext}^{1}(Y, S)=0$.

Since $\operatorname{Hom}(S, S)$ is non-zero and $\operatorname{Hom}(Y, S)$ is at most one-dimensional, we deduce that they are isomorphic and one-dimensional. In particular, $S$ is indecomposable. Moreover, the map $F$ is zero and hence $[S, S]^{1}=[X, S]$. We apply $\operatorname{Hom}(X,-)$ to $\xi$ and get the short exact sequence

$$
0 \longrightarrow \operatorname{Hom}(X, X) \longrightarrow \operatorname{Hom}(X, Y) \longrightarrow \operatorname{Hom}(X, S) \longrightarrow 0
$$

from which we deduce $[X, S]=[X, Y]-[X, X]=[X, Y]-1$ and the proof is complete.

\subsection{Quiver Grassmannians}

Let $Q$ be an acyclic quiver and $\mathbf{e} \leq \mathbf{d}$ be two dimension vectors for $Q$, where the partial order is componentwise. Let $M \in \mathrm{R}_{\mathbf{d}}(Q)$ be a representation $Q$ of dimension vector $\mathbf{d}$. The quiver Grassmannian $\operatorname{Gr}_{\mathbf{e}}(M)$ parametrizes the subrepresentations of $M$ of dimension vector e. To give the precise definition, we need to recall the construction of a family called the universal quiver Grassmannian (see [52], [12]). We define $\operatorname{Gr}_{\mathbf{e}}(\mathbf{d}):=$ $\prod \mathrm{Gr}_{e_{i}}\left(K^{d_{i}}\right)$. The universal quiver Grassmannian is the incidence variety

$$
\mathrm{Gr}_{\mathbf{e}}^{Q}(\mathbf{d}):=\left\{\left(\left(U_{i}\right),\left(M_{\alpha}\right)\right) \in \operatorname{Gr}_{\mathbf{e}}(\mathbf{d}) \times \mathrm{R}_{\mathbf{d}}(Q) \mid M_{\alpha}\left(U_{s(\alpha)}\right) \subseteq U_{t(\alpha)} \forall \alpha \in Q_{1}\right\}
$$

It is equipped with the two projections $\operatorname{Gr}_{\mathbf{e}}(\mathbf{d}) \prec \stackrel{p_{1}}{\longleftarrow} \operatorname{Gr}_{\mathbf{e}}^{Q}(\mathbf{d}) \stackrel{p_{2}}{\longrightarrow} \mathrm{R}_{\mathbf{d}}(Q)$ which are $G_{\mathbf{d}}$-equivariant. The map $p_{1}$ realizes $\operatorname{Gr}_{\mathbf{e}}^{Q}(\mathbf{d})$ as the total space of a homogeneous vector bundle over $\operatorname{Gr}_{\mathbf{e}}(\mathbf{d})$. This implies that it is irreducible, smooth of dimension $\langle\mathbf{e}, \mathbf{d}-\mathbf{e}\rangle+$ $\operatorname{dim} \mathrm{R}_{\mathbf{d}}(Q)$. The map $p_{2}$ is proper, and its image is the closed $G_{\mathbf{d}}$-stable subvariety of 
$\mathrm{R}_{\mathbf{d}}(Q)$ consisting of those representations admitting a subrepresentation of dimension vector e. The quiver Grassmannian $\operatorname{Gr}_{\mathbf{e}}(M)$ is defined to be the fiber of $p_{2}$ over the point $M$. In particular, if $M$ lies in the image of $p_{2}$, then every irreducible component of $\operatorname{Gr}_{\mathbf{e}}(M)$ has dimension at least $\langle\mathbf{e}, \mathbf{d}-\mathbf{e}\rangle$. In case there exists a rigid representation $M_{\mathbf{d}}$ of dimension vector $\mathbf{d}$, if the orbit of $M_{\mathbf{d}}$ lies in the image of $p_{2}$ then $p_{2}$ is surjective, and hence every representation $M \in \mathrm{R}_{\mathbf{d}}(Q)$ admits a subrepresentation of dimension vector e.

We will often use the universal families $\mathcal{U}$ and $\mathcal{Q}$ on $\operatorname{Gr}_{\mathbf{e}}(M)$. They arise as follows: let $\mathcal{U}_{i}$ be the pull-back of the universal rank $e_{i}$ subbundle of the trivial bundle with fiber $M_{i}$ on $\operatorname{Gr}_{e_{i}}\left(M_{i}\right)$ along the natural morphism $\operatorname{Gr}_{\mathbf{e}}(M) \rightarrow \operatorname{Gr}_{e_{i}}\left(M_{i}\right)$. Similarly let $\mathcal{Q}_{i}$ the pull-back of the universal rank $d_{i}-e_{i}$ quotient bundle. The family $\mathcal{U}=\left(\mathcal{U}_{i}\right)$ satisfies $M_{\alpha}\left(\mathcal{U}_{i}\right) \subseteq \mathcal{U}_{j}$. The fiber of $\mathcal{U}_{i}\left(\right.$ resp. $\left.\mathcal{Q}_{i}\right)$ over a subrepresentation $U$ is then canonically identified with the subspace $U_{i}$ of $M_{i}$ (resp. the quotient $M_{i} / U_{i}$ of $M_{i}$ ).

Sometimes it will be convenient to look at the set of $T$-valued points of the quiver Grassmannian $\operatorname{Gr}_{\mathbf{e}}(M)$, where $T$ is a scheme over the algebraically closed field $K$. That is, we consider the contravariant functor which assigns to any $K$-scheme $T$ the set $\operatorname{Hom}\left(T, \operatorname{Gr}_{\mathbf{e}}(M)\right):=\operatorname{Hom}_{\text {Spec } K}\left(T, \operatorname{Gr}_{\mathbf{e}}(M)\right)$. This functor is of course uniquely determined by its values on affine $K$-schemes. Let $T=\operatorname{Spec} A$. Recall that the set of $T$-valued points $\operatorname{Hom}\left(T, \operatorname{Gr}_{k}(V)\right)$ of the ordinary $\operatorname{Grassmannian} \mathrm{Gr}_{k}(V)$ of $k$-dimensional subspaces of an $n$-dimensional $K$-vector space $V$ is in functorial bijection to the set

\section{$\{U \mid U A$-submodule of $V \otimes A$ such that $(V \otimes A) / U$ is projective of rank $n-k\}$.}

The bijection is provided by the universal subbundle $\mathcal{U}$ on $\operatorname{Gr}_{k}(V)$ : to a morphism $f$ : $T \rightarrow \operatorname{Gr}_{k}(V)$ we assign the pull-back $f^{*} \mathcal{U}$ which corresponds to an $A$-module belonging to this set. Note that, as $(V \otimes A) / U$ is projective, the short exact sequence $0 \rightarrow U \rightarrow$ $V \otimes A \rightarrow(V \otimes A) / U \rightarrow 0$ splits and hence $U$ is also projective; its rank is $k$.

Using this universal property of the Grassmannian and the definition of the quiver Grassmannian, it is not hard to see that the set of $T$-valued points of $\operatorname{Gr}_{\mathbf{e}}(M)$ is the set of tuples $\left(U_{i}\right)$ of submodules $U_{i}$ of $M_{i} \otimes A$ for which $\left(M_{i} \otimes A\right) / U_{i}$ is projective of rank $d_{i}-e_{i}$ and such that $\left(M_{\alpha} \otimes \operatorname{id}_{A}\right)\left(U_{i}\right) \subseteq U_{j}$ holds for all $\alpha: i \rightarrow j$.

Given a $Q$-representation $M$ we can form the representation $M^{*}=D M$ of the opposite quiver $Q^{o p}$, and consider $\mathbf{e}^{*}=\operatorname{dim} M-\mathbf{e}$. Then there is an isomorphism of projective varieties induced by duality of Grassmannians: $\operatorname{Gr}_{\mathbf{e}}(M) \stackrel{\cong}{\rightarrow} \operatorname{Gr}_{\mathbf{e}^{*}}\left(M^{*}\right): N \mapsto(M / N)^{*}=$ $\operatorname{Ann}_{M^{*}}(N)$.

\subsection{Property (S) and cellular decomposition}

We recall some definitions of $[17$, Sec. 1$]$ :

Definition 9. A finite partition $\left(X_{i}\right)$ of a complex algebraic variety $X$ is said to be an $\alpha$-partition if 


$$
X_{1} \amalg X_{2} \amalg \cdots \amalg X_{i} \text { is closed in } X \text { for every } i \text {. }
$$

Clearly, every piece of an $\alpha$-partition is locally closed.

Definition 10. A cellular decomposition or affine paving of $X$ is an $\alpha$-partition into parts $X_{i}$ which are isomorphic to affine spaces.

Let $X$ be a complex variety. We denote by $H_{i}(X)=H_{i}^{\mathrm{BM}}(X(\mathbb{C}) ; \mathbb{Z})$ the Borel-Moore homology with integer coefficients of $X$ equipped with the analytic topology.

Definition 11. The variety $X$ has property $(S)$ if

(1) numerical and rational equivalence on $X$ coincide,

(2) $H_{i}(X)=0$ for $i$ odd, and

(3) the cycle map $\varphi_{i}: A_{i}(X) \rightarrow H_{2 i}(X)$ is an isomorphism for all $i$.

(See $[29,1.3]$ for the definition of the Chow groups $A_{i}(X)$ and $[29,19.1]$ for the cycle $\operatorname{map} \varphi_{i}$.) Property $(\mathrm{S})$ was introduced in [17] as a replacement of cellular decomposition. Indeed, if a variety admits an $\alpha$-partition into pieces having property $(\mathrm{S})$, then it has property (S) [17, Lem. 1.8]. In particular, cellular decomposition implies property (S). Springer fibers for classical groups admit a cellular decomposition, and for the exceptional groups have property $(\mathrm{S})$ [17, Th. 3.9]. It is still open whether Springer fibers of exceptional groups admit a cellular decomposition [38, Pag. 32-33].

The fact that numerical and rational equivalence on $X$ agree implies that $A_{*}(X)$ is a finitely generated free abelian group [29, Ex. 19.1.4]. If $n$ is the complex dimension of $X$ then $A^{n-i}(X)=A_{i}(X)$. Supposing that $X$ is smooth, the Borel-Moore homology group $H_{i}(X)$ equals the singular cohomology group $H^{2 n-i}(X)$. So for a smooth complex variety $X$ having property $(\mathrm{S})$, the odd-degree cohomology groups $H^{2 i+1}(X)$ vanish and the cycle map $A^{i}(X) \rightarrow H^{2 i}(X)$ is an isomorphism.

The following well-known example shows that even if a variety admits a partition into affine spaces, it is not necessarily true that it admits a cellular decomposition.

Example 12. Let $X=\left\{[x: y: z] \in \mathbf{P}^{2} \mid x y z=0\right\}$ be the union of three $\mathbf{P}^{1}$ which meet pairwise in distinct points. Then $X=\mathbf{A}^{1} \amalg \mathbf{A}^{1} \amalg \mathbf{A}^{1}$ but $H^{1}(X)$ is clearly nonzero and hence $X$ does not have property $(\mathrm{S})$. In particular, it cannot admit a cellular decomposition.

We often use the following lemma to deduce that cellular decompositions "lift" along affine bundles.

Lemma 13. Let $p: A \rightarrow X$ be an affine bundle. If $X$ admits a cellular decomposition or $X$ has property $(S)$, then so does $A$. 
Proof. For property $(\mathrm{S})$, this is [17, Lem. 1.9]. If $\left(X_{i}\right)$ is a cellular decomposition of $X$, then, by the Quillen-Suslin theorem [45, Th. 4], $\left(A_{i}:=p^{-1}\left(X_{i}\right)\right)$ is a cellular decomposition of $A$.

Definition 14. We say that a quiver representation has property $(\mathrm{C})$ (resp. $(\mathrm{S})$ ) if every non-empty quiver Grassmannian associated with it admits a cellular decomposition (resp. has property $(\mathrm{S})$ ).

\subsection{Quasi-simple representations}

The results of this section will only be needed in Section 6.2, for the study of quiver Grassmannians attached to preprojective representations of affine quivers. Thus, for simplicity, throughout the section, $Q$ denotes an acyclic, connected and finite quiver without multiple arrows. In analogy with Ringel [46] we give the following definition.

Definition 15. An indecomposable $Q$-representation $S$ is quasi-simple if there are no irreducible monomorphisms ending in $S$ and there are no irreducible epimorphisms starting from $S$.

Proposition 16. An indecomposable $Q$-representation $M$ is quasi-simple if and only if $M$ is either simple or an almost split sequence ending in or starting from $M$ has indecomposable middle term.

Proof. Let $M$ be quasi-simple, non-projective and non-injective. If $M$ is regular, then the claim follows from [46]. By duality, we can assume that $M$ is preprojective. Let $E_{1}$ and $E_{2}$ be two non-isomorphic indecomposable direct summands of the middle term $E$ of the almost split sequence ending in $M$. The composite map $E_{1} \longrightarrow M C \tau^{-} E_{2}$ is neither surjective nor injective. This contradicts the Happel-Ringel Lemma 7 and thus $E$ is indecomposable since $Q$ has no multiple arrows.

We can give another characterization of quasi-simple modules. Recall that a leaf of $Q$ is a vertex $i \in Q_{0}$ which is joined to precisely one vertex by an edge of $Q$.

Corollary 17. The $Q$-representation $M=\tau^{-t} P_{k}$ is quasi-simple if and only if $k$ is a leaf of $Q$.

Proof. It follows by the construction of almost split sequences via the knitting algorithm.

If $S$ is either regular or preprojective there is a simpler characterization of quasisimplicity. 
Corollary 18. An indecomposable, not preinjective, representation $S$ of a non-Dynkin quiver is quasi-simple if and only if there are no irreducible monomorphisms ending in $S$.

Proof. If $S$ is regular this is well-known [46]. Let $S$ be preprojective without irreducible monomorphisms ending in $S$. Suppose that there is an irreducible epimorphism $S \longrightarrow F$ starting from $S$. Then the composition $\tau F \longrightarrow S \longrightarrow F$ is epi. Hence, $\operatorname{dim} F>$ $\operatorname{dim} \tau^{-k} F$ for every $k$, since $\tau^{-}$preserves epis. By hypothesis $Q$ is not Dynkin, thus we get a contradiction.

Lemma 19. Let $\iota: X \hookrightarrow Y$ be an irreducible monomorphism between preprojective $Q$ representations and let $S=$ Coker $(\iota)$. Then $S$ is indecomposable, rigid and $[S, X]^{1}=1$. Moreover there are no irreducible monomorphisms ending in $S$. If $S$ is either regular or preprojective then $S$ is quasi-simple.

Proof. Follows from Unger's Lemma 8 and Corollary 18.

\section{Decomposition of quiver Grassmannians induced by short exact sequences}

Let $Q$ be a quiver and let $\eta: 0 \longrightarrow M^{\prime} \stackrel{\iota}{\longrightarrow} M \stackrel{\pi}{\longrightarrow} M^{\prime \prime} \longrightarrow 0$ be a short exact sequence in $\operatorname{Rep}(Q)$. Let $\mathbf{e} \in \mathbb{Z}_{\geq 0}^{Q_{0}}$ be a dimension vector. We consider the canonical map

$$
\Psi^{\eta}: G r_{\mathbf{e}}(M) \rightarrow \coprod_{\mathbf{f}+\mathbf{g}=\mathbf{e}} G r_{\mathbf{f}}\left(M^{\prime}\right) \times G r_{\mathbf{g}}\left(M^{\prime \prime}\right): N \mapsto\left(\iota^{-1}(N), \pi(N)\right)
$$

It induces subsets $\mathcal{S}_{\mathbf{f}, \mathbf{g}}^{\eta}:=\left(\Psi^{\eta}\right)^{-1}\left(\operatorname{Gr}_{\mathbf{f}}\left(M^{\prime}\right) \times \operatorname{Gr}_{\mathbf{g}}\left(M^{\prime \prime}\right)\right)$ in $\operatorname{Gr}_{\mathbf{e}}(M)$ and a decomposition

$$
\operatorname{Gr}_{\mathbf{e}}(M)=\coprod_{\mathbf{f}+\mathbf{g}=\mathbf{e}} \mathcal{S}_{\mathbf{f}, \mathbf{g}}^{\eta}
$$

Lemma 20. The partition (6) is an $\alpha$-partition of $\operatorname{Gr}_{\mathbf{e}}(M)$. In particular if $\mathcal{S}_{\mathbf{f}, \mathbf{g}}^{\eta}$ admits a cellular decomposition for any $\mathbf{f}+\mathbf{g}=\mathbf{e}$ then the same holds for $\operatorname{Gr}_{\mathbf{e}}(M)$.

Proof. Clearly the partition (6) is finite. The function $\operatorname{Gr}_{\mathbf{e}}(M) \rightarrow \mathbb{Z}_{\geq 0}^{Q_{0}}: N \mapsto \operatorname{dim}(N \cap$ $\operatorname{ker}(\pi)$ ) which maps $N \in \operatorname{Gr}_{\mathbf{e}}(M)$ to the dimension vector of $N \cap \operatorname{ker}(\pi)$ is uppersemicontinuous. In other words for every $\mathbf{n} \in \mathbb{Z}_{\geq 0}^{Q_{0}}$ the union of strata $\amalg_{\mathbf{f} \geq \mathbf{n}} \mathcal{S}_{\mathbf{f}, \mathbf{g}}^{\eta} \subset$ $\operatorname{Gr}_{\mathbf{e}}(M)$ is closed in $\operatorname{Gr}_{\mathbf{e}}(M)$. By totally ordering the strata $\mathcal{S}_{\mathbf{f}, \mathbf{g}}^{\eta}$ compatibly with this partial ordering, we get the statement.

For any f, $\mathbf{g}$, the restricted map $\Psi_{\mathbf{f}, \mathbf{g}}^{\eta}: \mathcal{S}_{\mathbf{f}, \mathbf{g}}^{\eta} \rightarrow \operatorname{Gr}_{\mathbf{f}}\left(M^{\prime}\right) \times \operatorname{Gr}_{\mathbf{g}}\left(M^{\prime \prime}\right)$ is algebraic.

Lemma 21. A point $\left(N^{\prime}, N^{\prime \prime}\right) \in \operatorname{Gr}_{\mathbf{f}}\left(M^{\prime}\right) \times \operatorname{Gr}_{\mathbf{g}}\left(M^{\prime \prime}\right)$ lies in the image of $\Psi_{\mathbf{f}, \mathbf{g}}^{\eta}$ if and only if $\eta$ is in the kernel of the canonical map $\operatorname{Ext}^{1}\left(M^{\prime \prime}, M^{\prime}\right) \rightarrow \operatorname{Ext}^{1}\left(N^{\prime \prime}, M^{\prime} / N^{\prime}\right)$. 
Proof. Given $\left(N^{\prime}, N^{\prime \prime}\right)$, the image $\bar{\eta}$ of $\eta$ under the composition of the canonical maps

$$
\eta \in \operatorname{Ext}^{1}\left(M^{\prime \prime}, M^{\prime}\right) \longrightarrow \operatorname{Ext}^{1}\left(N^{\prime \prime}, M^{\prime}\right) \longrightarrow \operatorname{Ext}^{1}\left(N^{\prime \prime}, M^{\prime} / N^{\prime}\right) \ni \bar{\eta}
$$

corresponds to the extension $0 \rightarrow M^{\prime} / N^{\prime} \rightarrow \bar{N} / N^{\prime} \rightarrow N^{\prime \prime} \rightarrow 0$, where $\bar{N}=\pi^{-1}\left(N^{\prime \prime}\right)$.

Now $\bar{\eta}=0$ if and only if there exists a subrepresentation $N$ of $\bar{N}$, containing $N^{\prime}$, projecting to $N^{\prime \prime}$ via $\pi$ and such that $M^{\prime} \cap N=N^{\prime}$. These are precisely the conditions defining the image of $\Psi_{\mathbf{f}, \mathbf{g}}^{\eta}$.

Let us consider the forgetful functor $F: \operatorname{Rep}(Q) \rightarrow \operatorname{Rep}\left(Q_{0}\right)$ which associates to a $Q$-representation $M$ the $Q_{0}$-graded vector space $M_{0}:=\bigoplus_{i \in Q_{0}} M_{i}$. By definition, a quiver Grassmannian $\operatorname{Gr}_{\mathbf{e}}(M)$ admits a closed embedding $\operatorname{Gr}_{\mathbf{e}}(M) \rightarrow \prod_{i \in Q_{0}} \operatorname{Gr}_{e_{i}}\left(M_{i}\right)=$ $\operatorname{Gr}_{\mathbf{e}}\left(M_{0}\right)$. We denote by $\mathcal{U}_{i}$ (resp. $\mathcal{Q}_{i}$ ) the pull-back of the tautological subbundle (resp. quotient bundle) of the trivial vector bundle $M_{i}$ on $\operatorname{Gr}_{e_{i}}\left(M_{i}\right)$ along the projection $\prod_{j} \mathrm{Gr}_{e_{j}}\left(M_{j}\right) \rightarrow \mathrm{Gr}_{e_{i}}\left(M_{i}\right)$. As in Section 2.3, we denote the pullback of $\mathcal{U}_{i}$ (resp. $\mathcal{Q}_{i}$ ) along this embedding by the same name. The fiber of $\mathcal{U}_{i}$ (resp. $\mathcal{Q}_{i}$ ) over a subrepresentation $U$ is then canonically identified with the subspace $U_{i}$ (resp. the quotient $M_{i} / U_{i}$ ) of $M_{i}$. We consider the following vector bundles on $\operatorname{Gr}_{\mathbf{f}}\left(M_{0}^{\prime}\right) \times \operatorname{Gr}_{\mathbf{g}}\left(M_{0}^{\prime \prime}\right)$ (see section 2.3 for the definition of the various objects involved):

$$
H_{0}:=\bigoplus_{i} \underline{\operatorname{Hom}}\left(p_{2}^{*} \mathcal{U}_{i}^{\prime \prime}, p_{1}^{*} \mathcal{Q}_{i}^{\prime}\right), \quad K_{0}:=\bigoplus_{\alpha} \underline{\operatorname{Hom}}\left(p_{2}^{*} \mathcal{U}_{s(\alpha)}^{\prime \prime}, p_{1}^{*} \mathcal{Q}_{t(\alpha)}^{\prime}\right)
$$

where $p_{1}: \operatorname{Gr}_{\mathbf{f}}\left(M_{0}^{\prime}\right) \times \operatorname{Gr}_{\mathbf{g}}\left(M_{0}^{\prime \prime}\right) \rightarrow \operatorname{Gr}_{\mathbf{f}}\left(M_{0}^{\prime}\right)$ and $p_{2}: \operatorname{Gr}_{\mathbf{g}}\left(M_{0}^{\prime}\right) \times \operatorname{Gr}_{\mathbf{g}}\left(M_{0}^{\prime \prime}\right) \rightarrow \operatorname{Gr}_{\mathbf{g}}\left(M_{0}^{\prime \prime}\right)$ are the projections onto the two factors and Hom denotes the vector bundle of homomorphisms. Define the bundles $H$ and $K$ on $\operatorname{Gr}_{\mathbf{f}}\left(M^{\prime}\right) \times \operatorname{Gr}_{\mathbf{g}}\left(M^{\prime \prime}\right)$ as the restrictions

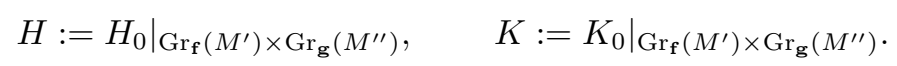

Let $\Phi: H \rightarrow K$ be the map of vector bundles defined over a point $\left(N^{\prime}, N^{\prime \prime}\right)$ as the Ringel linear map (2):

$$
\Phi_{\left(N^{\prime}, N^{\prime \prime}\right)}: \bigoplus_{i} \operatorname{Hom}\left(N_{i}^{\prime \prime}, M_{i}^{\prime} / N_{i}^{\prime}\right) \rightarrow \bigoplus_{\alpha: i \rightarrow j} \operatorname{Hom}\left(N_{i}^{\prime \prime}, M_{j}^{\prime} / N_{j}^{\prime}\right)
$$

which sends a tuple $f=\left(f_{i}\right)_{i}$ of linear maps $f_{i}: N_{i}^{\prime \prime} \rightarrow M_{i}^{\prime} / N_{i}^{\prime}$ to $\Phi_{N^{\prime \prime}}^{M^{\prime} / N^{\prime}}\left(\left(f_{i}\right)_{i}\right)=$ $\left(Q_{\alpha}^{\prime} f_{i}-f_{j} N_{\alpha}^{\prime \prime}\right)_{\alpha: i \rightarrow j}$ (see (2)). In this context, $N_{\alpha}^{\prime \prime}: N_{i}^{\prime \prime} \rightarrow N_{j}^{\prime \prime}$ is the restriction of $M_{\alpha}^{\prime \prime}$ and $Q_{\alpha}^{\prime}: M_{i}^{\prime} / N_{i}^{\prime} \rightarrow M_{j}^{\prime} / N_{j}^{\prime}$ is the map induced by $M_{\alpha}^{\prime}$ between the quotients. We briefly write: $f \mapsto(f \alpha-\alpha f)_{\alpha}$. Note that over a point $\left(N^{\prime}, N^{\prime \prime}\right) \in \operatorname{Gr}_{\mathbf{f}}\left(M^{\prime}\right) \times \operatorname{Gr}_{\mathbf{g}}\left(M^{\prime \prime}\right)$, the kernel of $\Phi_{\left(N^{\prime}, N^{\prime \prime}\right)}$ is $\operatorname{Hom}_{Q}\left(N^{\prime \prime}, M^{\prime} / N^{\prime}\right)$ while its cokernel is $\operatorname{Ext}_{Q}^{1}\left(N^{\prime \prime}, M^{\prime} / N^{\prime}\right)$ (see Section 2.1). Let $\eta_{0}=F(\eta)$ be the short exact sequence of $Q_{0}$-graded vector spaces, together with a splitting $\theta$ : 


$$
\eta_{0}: 0 \longrightarrow M_{0}^{\prime} \stackrel{\iota}{\longrightarrow} M_{0} \stackrel{\theta}{\stackrel{\theta}{\longrightarrow}} M_{0}^{\prime \prime} \longrightarrow 0
$$

The splitting $\theta$ induces a section $z_{\theta}=\left(z_{\theta, \alpha}\right)_{\alpha \in Q_{1}}$ of the vector bundle $K$ defined by $z_{\theta, \alpha}=\overline{\alpha \theta-\theta \alpha}$. Here, for a morphism $g: N^{\prime \prime} \rightarrow M^{\prime}$ we denote by $\bar{g}: N^{\prime \prime} \rightarrow M^{\prime} / N^{\prime}$ the induced morphism on the quotient. Note that, given any $\left(N^{\prime}, N^{\prime \prime}\right) \in \operatorname{Gr}_{\mathbf{f}}\left(M^{\prime}\right) \times \operatorname{Gr}_{\mathbf{g}}\left(M^{\prime \prime}\right)$, the image of $z_{\theta}\left(N^{\prime}, N^{\prime \prime}\right)$ under the map $K_{\left(N^{\prime}, N^{\prime \prime}\right)} \rightarrow \operatorname{Ext}_{Q}^{1}\left(N^{\prime \prime}, M^{\prime} / N^{\prime}\right)$ agrees with the image of $\eta$ under $\operatorname{Ext}_{Q}^{1}\left(M^{\prime \prime}, M^{\prime}\right) \rightarrow \operatorname{Ext}_{Q}^{1}\left(N^{\prime \prime}, M^{\prime} / N^{\prime}\right)$.

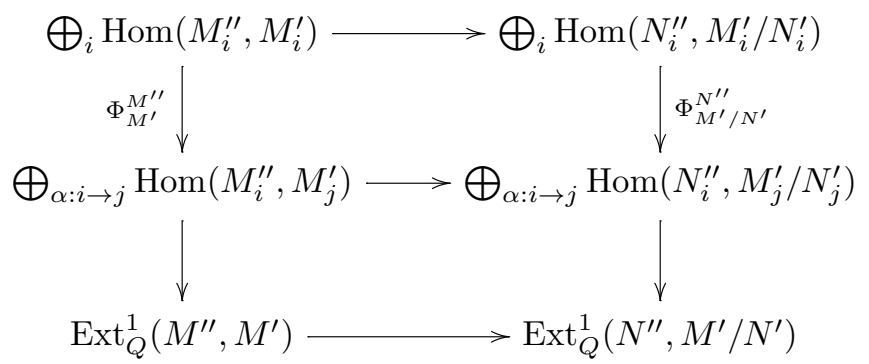

Lemma 22. There is an isomorphism $\mathcal{S}_{\mathbf{f}, \mathbf{g}}^{\eta} \cong \Phi^{-1}\left(z_{\theta}\right)$ of schemes over $\operatorname{Gr}_{\mathbf{f}}\left(M^{\prime}\right) \times$ $\operatorname{Gr}_{\mathbf{g}}\left(M^{\prime \prime}\right)$.

Proof. We show that the splitting $\theta$ induces an isomorphism

$$
H_{0} \stackrel{\cong}{\longrightarrow} \mathcal{S}_{\mathbf{f}, \mathbf{g}}^{\eta_{0}}
$$

as schemes over $X_{0}:=\operatorname{Gr}_{\mathbf{f}}\left(M_{0}^{\prime}\right) \times \operatorname{Gr}_{\mathbf{g}}\left(M_{0}^{\prime \prime}\right)$. We construct this isomorphism by defining a functorial bijection between the sets $\operatorname{Hom}_{X_{0}}\left(T, H_{0}\right)$ and $\operatorname{Hom}_{X_{0}}\left(T, \mathcal{S}_{\mathbf{f}, \mathbf{g}}^{\eta_{0}}\right)$. Let $T=$ Spec $A$ be an affine $X_{0^{-}}$-scheme. As explained in subsection 2.3 the morphism $T \rightarrow X_{0}$ corresponds to a pair $\left(N^{\prime}, N^{\prime \prime}\right)$ of $A Q_{0}$-submodules of $N^{\prime} \subseteq M_{0}^{\prime} \otimes A$ and $N^{\prime \prime} \subseteq M_{0}^{\prime \prime} \otimes A$ for which $\left(M_{i}^{\prime} \otimes A\right) / N_{i}^{\prime}$ is a projective $A$-module of rank $\operatorname{dim} M_{i}^{\prime}-f_{i}$ and $\left(M_{i}^{\prime \prime} \otimes A\right) / N_{i}^{\prime \prime}$ is projective of rank $\operatorname{dim} M_{i}^{\prime \prime}-g_{i}$. We get

$$
\begin{aligned}
\operatorname{Hom}_{X_{0}}\left(T, H_{0}\right)= & \operatorname{Hom}_{A Q_{0}}\left(N^{\prime \prime},\left(M_{0}^{\prime} \otimes A\right) / N^{\prime}\right) \\
\operatorname{Hom}_{X_{0}}\left(T, \mathcal{S}_{\mathbf{f}, \mathbf{g}}^{\eta_{0}}\right)= & \left\{N \subseteq M_{0} \otimes A \mid\left(M_{i} \otimes A\right) / N_{i} \text { proj. of rk. } d_{i}-e_{i}, \iota^{-1}(N)=N^{\prime}\right. \\
& \text { and } \left.\pi(N)=N^{\prime \prime}\right\} .
\end{aligned}
$$

Here we denote the base extensions of $\iota$ and $\pi$ to maps $M_{0}^{\prime} \otimes A \rightarrow M_{0} \otimes A \rightarrow M_{0}^{\prime \prime} \otimes A$ also by $\iota$ resp. $\pi$. We send a point $f \in \operatorname{Hom}_{X_{0}}\left(T, H_{0}\right)$ to the subspace

$$
N_{f}=\left\{\iota\left(m^{\prime}\right)+\theta\left(n^{\prime \prime}\right) \mid m^{\prime} \in M_{0}^{\prime} \otimes A \text { and } n^{\prime \prime} \in N^{\prime \prime} \text { such that } m^{\prime}+N^{\prime}=f\left(n^{\prime \prime}\right)\right\} .
$$

A word on a piece of notation used in the above equation: by $m^{\prime}+N^{\prime}$ we denote the image of $m^{\prime} \in M_{0}^{\prime} \otimes A$ under the quotient map $M_{0}^{\prime} \otimes A \rightarrow\left(M_{0}^{\prime} \otimes A\right) / N^{\prime}$. It is obvious 
that $\iota^{-1}\left(N_{f}\right)=N^{\prime}$ and $\pi\left(N_{f}\right)=N^{\prime \prime}$, so $N_{f}$ lies in $\operatorname{Hom}_{X_{0}}\left(T, \mathcal{S}_{\mathbf{f}, \mathbf{g}}^{\eta_{0}}\right)$. The inverse of this association is given by mapping a subspace $N$ in $\operatorname{Hom}_{X_{0}}\left(T, \mathcal{S}_{\mathbf{f}, \mathbf{g}}^{\eta_{0}}\right)$ to the map $f_{N}: N^{\prime \prime} \rightarrow$ $\left(M_{0}^{\prime} \otimes A\right) / N^{\prime}$ which is defined by

$$
f_{N}\left(n^{\prime \prime}\right)=m^{\prime}+N^{\prime}
$$

where $m^{\prime} \in M_{0}^{\prime} \otimes A$ is an element for which $\iota\left(m^{\prime}\right)+\theta\left(n^{\prime \prime}\right) \in N$. We show that the map $f_{N}$ is well-defined. To show the existence of such an element $m^{\prime}$ we choose, for a given $n^{\prime \prime} \in N^{\prime \prime}$, an inverse image $n \in N$ under $\pi$. Then, as $\theta$ is a splitting, there exists a unique $m^{\prime} \in M_{0}^{\prime} \otimes A$ such that $\iota\left(m^{\prime}\right)+\theta\left(n^{\prime \prime}\right)=n$. Now assume $m_{1}^{\prime}, m_{2}^{\prime} \in M_{0}^{\prime} \otimes A$ such that $\iota\left(m_{1}^{\prime}\right)+\theta\left(n^{\prime \prime}\right)$ and $\iota\left(m_{2}^{\prime}\right)+\theta\left(n^{\prime \prime}\right)$ both lie in $N$. Then so does $\iota\left(m_{1}^{\prime}-m_{2}^{\prime}\right)$ and hence $m_{1}^{\prime}-m_{2}^{\prime} \in \iota^{-1}(N)=N^{\prime}$. The map $f_{N}$ is hence well-defined. It is easy to see that these two associations are mutually inverse.

Now suppose that $T \rightarrow X_{0}$ factors through $X=\operatorname{Gr}_{\mathbf{f}}\left(M^{\prime}\right) \times \operatorname{Gr}_{\mathbf{g}}\left(M^{\prime \prime}\right)$. That means the corresponding pair $\left(N^{\prime}, N^{\prime \prime}\right)$ of $A Q_{0}$-modules is actually a pair of $A Q$-submodules of $M^{\prime} \otimes A$ and $M^{\prime \prime} \otimes A$, respectively. An element $f \in \operatorname{Hom}_{X_{0}}\left(T, H_{0}\right)=\operatorname{Hom}_{X}(T, H)$ lies in $\operatorname{Hom}_{X}\left(T,\left.\Phi^{-1}\left(z_{\theta}\right)\right|_{X}\right)$ if and only if $f \alpha-\alpha f=z_{\theta}$. A point $N \in \operatorname{Hom}_{X_{0}}\left(T, \mathcal{S}_{\mathbf{f}, \mathbf{g}}^{\eta_{0}}\right)=$ $\operatorname{Hom}_{X}\left(T,\left.\mathcal{S}_{\mathbf{f}, \mathbf{g}}^{\eta_{0}}\right|_{X}\right)$ is contained in $\operatorname{Hom}_{X}\left(T, \mathcal{S}_{\mathbf{f}, \mathbf{g}}^{\eta}\right)$ if and only if $\alpha N \subseteq N$. The bijections above restrict to a bijection $\operatorname{Hom}_{X}\left(T, \Phi^{-1}\left(z_{\theta}\right)\right) \rightarrow \operatorname{Hom}_{X}\left(T, \mathcal{S}_{\mathbf{f}, \mathbf{g}}^{\eta}\right)$. We have shown that there exists an isomorphism

$$
\Phi^{-1}\left(z_{\theta}\right) \stackrel{\cong}{\longrightarrow} \mathcal{S}_{\mathbf{f}, \mathbf{g}}^{\eta}
$$

of schemes over $\operatorname{Gr}_{\mathbf{f}}\left(M^{\prime}\right) \times \operatorname{Gr}_{\mathbf{g}}\left(M^{\prime \prime}\right)$.

Lemma 23. Let $X$ be a scheme, let $H$ and $K$ be two vector bundles on $X$, let $\varphi: H \rightarrow K$ be a homomorphism of vector bundles, and let $s$ be a global section of $K$ whose image under the map $H^{0}(X, K) \rightarrow H^{0}(X, \operatorname{coker}(\varphi))$ is zero. Then the inverse image $\varphi^{-1}(s)$ has the structure of a torsor over $X$ for $\operatorname{ker} \varphi$.

Proof. We use in the proof the abbreviations $E=\operatorname{ker} \varphi$ and $P=\varphi^{-1}(s)$. There is a cartesian square

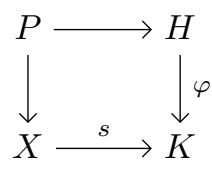

Let $T$ be a scheme over $X$. Then $\operatorname{Hom}_{X}(T, P)$ is the set of all $f \in \operatorname{Hom}_{X}(T, H)$ for which $\varphi \circ f=\left.s\right|_{T}$. On the other hand $\operatorname{Hom}_{X}(T, E)$ is the subgroup of all $g \in \operatorname{Hom}_{X}(T, H)$ such that $\varphi \circ g=0$ in the abelian group $\operatorname{Hom}_{X}(T, K)$. The additive group of $\operatorname{Hom}_{X}(T, E)$ acts on $\operatorname{Hom}_{X}(T, P)$ via $(g, f) \mapsto g+f$, where the addition is taken in the abelian group $\operatorname{Hom}_{X}(T, H)$. To show that $P$ is a torsor for $E$ it suffices to find an open cover $\left\{U_{i}\right\}_{i}$ 
of $X$ such that $\operatorname{Hom}_{X}\left(U_{i}, P\right) \neq \emptyset$ for every $i$. By assumption $s$ is mapped to zero under $H^{0}(X, K) \rightarrow H^{0}(X, \operatorname{coker}(\varphi))$. That means $s$ lies in $H^{0}(X, \operatorname{im}(\varphi))$. So we find an open cover $\left\{U_{i}\right\}_{i}$ of $X$ and sections $s_{i}^{\prime} \in H^{0}\left(U_{i}, H\right)$ with $\varphi \circ s_{i}^{\prime}=\left.s\right|_{U_{i}}$ for every $i$. Using the cartesian diagram above we get a unique morphism $r_{i}: U_{i} \rightarrow P$ such that

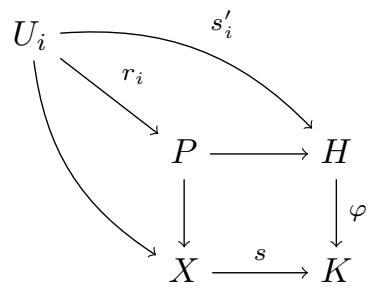

is commutative. The map $U_{i} \rightarrow X$ in the above diagram is an open immersion. We have found $r_{i} \in \operatorname{Hom}_{X}\left(U_{i}, P\right)$. This implies that $P$ is a torsor for $E$.

Combining Lemmas 21, 22, and 23 we are able to show

Theorem 24. Let $Y$ be a locally closed reduced subscheme of $\operatorname{Gr}_{\mathbf{f}}\left(M^{\prime}\right) \times \operatorname{Gr}_{\mathbf{g}}\left(M^{\prime \prime}\right)$ which lies in the image of $\Psi_{\mathbf{f}, \mathbf{g}}^{\eta}$. Then $\left.\left(\mathcal{S}_{\mathbf{f}, \mathbf{g}}^{\eta}\right)\right|_{Y}$ is a torsor for $\left.(\operatorname{ker} \Phi)\right|_{Y}$. In particular, if the dimension of $\operatorname{Hom}_{Q}\left(N^{\prime \prime}, M^{\prime} / N^{\prime}\right)$ is constant, say equal to $d$, for all closed points $\left(N^{\prime}, N^{\prime \prime}\right)$ of $Y$ then $\left.\left(\mathcal{S}_{\mathbf{f}, \mathbf{g}}\right)\right|_{Y}$ is an affine bundle on $Y$ of rank $d$.

Proof. Consider the section $\left.z_{\theta}\right|_{Y}$ of $\left.K\right|_{Y}$ and its image $t$ in $\left.(\operatorname{coker} \Phi)\right|_{Y}$. From Lemma 21 we deduce that $t(y)=0$ for every $K$-valued point of $Y$. As $Y$ is reduced it follows that $t=0$. We are able to apply Lemma 23 to $\Phi^{-1}\left(\left.z_{\theta}\right|_{Y}\right)$. Together with Lemma 22 , the first claim follows. For the second claim, note that, as $Y$ is reduced, $\left.(\operatorname{ker} \Phi)\right|_{Y}$ is a vector bundle if $\left[N^{\prime \prime}, M^{\prime} / N^{\prime}\right]$ is constant.

\subsection{Generating extensions}

In this section we analyze a class of extensions for which the fiber dimension of $\Psi_{\mathbf{f}, \mathbf{g}}^{\xi}$ is precisely equal to $\left\langle\mathbf{g}, \operatorname{dim} M^{\prime}-\mathbf{f}\right\rangle$. We call them generating extensions. They are defined as follows.

Definition 25. Let $X$ and $S$ be two representations of the quiver $Q$. We say that an extension $\xi \in \operatorname{Ext}^{1}(S, X)$ is generating if it is a generator of the vector space $\operatorname{Ext}^{1}(S, X)$.

By definition, a generating extension $\xi \in \operatorname{Ext}^{1}(S, X)$ is either split (in which case $\left.[S, X]^{1}=0\right)$ or it is non-split and $[S, X]^{1}=1$.

Theorem 26. Let $\xi \in \operatorname{Ext}^{1}(S, X)$ be a generating extension. Then the image of $\Psi_{\mathbf{f}, \mathbf{g}}^{\xi}$ is the open subset of $\operatorname{Gr}_{\mathbf{f}}(X) \times \operatorname{Gr}_{\mathbf{g}}(S)$ given by $\operatorname{Im}\left(\Psi_{\mathbf{f}, \mathbf{g}}^{\xi}\right)=\left\{\left(N^{\prime}, N^{\prime \prime}\right) \mid\left[N^{\prime \prime}, X / N^{\prime}\right]^{1}=0\right\}$ and $\mathcal{S}_{\mathbf{f}, \mathbf{g}}^{\xi}$ is an affine bundle of rank $\langle\mathbf{g}, \operatorname{dim} X-\mathbf{f}\rangle$ over it. 
Proof. Let $\mathcal{Y}$ be the image of $\Psi_{\mathbf{f}, \mathbf{g}}^{\xi}$. Since $\xi$ is generating, the description of $\mathcal{Y}$ follows immediately from Lemma 21. Since Coker $\Phi_{\left(N^{\prime}, N^{\prime \prime}\right)} \cong \operatorname{Ext}^{1}\left(N^{\prime \prime}, X / N^{\prime}\right)$, it follows that $\mathcal{Y}$ is precisely the locus where the map $\Phi: H \rightarrow K$ is surjective. By Lemma $22, \mathcal{S}_{\mathbf{f}, \mathbf{g}}^{\xi} \cong$ $\Phi^{-1}\left(z_{\theta}\right)$ on $\mathcal{Y}$. Since $\Phi$ is surjective on $\mathcal{Y}$, for every point $y \in \mathcal{Y}$ there exists an open neighborhood $U$ such that $z_{\theta} \mid U$ is in the image of the map $H^{0}(U, H) \rightarrow H^{0}(U, K)$. We can hence use Lemma 23 to deduce that, locally around every point of $\mathcal{Y}, \Phi^{-1}\left(z_{\theta}\right)$ is a trivial affine bundle for $\operatorname{Ker}(\Phi)$.

In case $\xi=0$ is split, then $\Psi_{\mathbf{f}, \mathbf{g}}^{\xi}$ is surjective and $\mathcal{S}_{\mathbf{f}, \mathbf{g}}^{\xi}$ is an affine bundle with a global section isomorphic to $\operatorname{Ker}(\Phi)$. In the next section we treat the case of a non-split generating extension.

\subsection{Non-split generating extensions}

In this section we provide a more precise description of the image of the map $\Psi_{\mathbf{f}, \mathbf{g}}^{\xi}$ for a generating extension $\xi \in \operatorname{Ext}^{1}(S, X)$ which is non-split. Throughout the section we denote the middle term of $\xi$ by $Y$.

\section{Lemma 27.}

(1) Let $N, N^{\prime} \subset X$ such that $[S, X / N]^{1}=\left[S, X / N^{\prime}\right]^{1}=1$. Then $\left[S, X /\left(N+N^{\prime}\right)\right]^{1}=1$.

(2) Let $N, N^{\prime} \subset S$ such that $[N, X]^{1}=\left[N^{\prime}, X\right]^{1}=1$. Then $\left[N \cap N^{\prime}, X\right]^{1}=1$.

Proof. From $0 \rightarrow \frac{X}{N \cap N^{\prime}} \rightarrow \frac{X}{N} \oplus \frac{X}{N^{\prime}} \rightarrow \frac{X}{N+N^{\prime}} \rightarrow 0$ we get the exact sequence

$$
\operatorname{Ext}^{1}\left(S, \frac{X}{N \cap N^{\prime}}\right) \rightarrow \operatorname{Ext}^{1}\left(S, \frac{X}{N}\right) \oplus \operatorname{Ext}^{1}\left(S, \frac{X}{N^{\prime}}\right) \rightarrow \operatorname{Ext}^{1}\left(S, \frac{X}{N+N^{\prime}}\right) \rightarrow 0
$$

and from $0 \rightarrow N \cap N^{\prime} \rightarrow N \oplus N^{\prime} \rightarrow N+N^{\prime} \rightarrow 0$ we get the exact sequence

$$
\operatorname{Ext}^{1}\left(N+N^{\prime}, X\right) \rightarrow \operatorname{Ext}^{1}(N, X) \oplus \operatorname{Ext}^{1}\left(N^{\prime}, X\right) \rightarrow \operatorname{Ext}^{1}\left(N \cap N^{\prime}, X\right) \rightarrow 0 .
$$

In the above exact sequences the middle term has dimension two by hypothesis, and the extreme terms have dimension at most one.

In view of Lemma 27 we can give the following key definition.

Definition 28. Let $X$ and $S$ be such that $[S, X]^{1}=1$. We define the following subrepresentations:

$$
X_{S}:=\max \left\{N \subset X \mid[S, X / N]^{1}=1\right\} \subset X, \quad \text { and } \quad S^{X}:=\min \left\{N \subset S \mid[N, X]^{1}=1\right\} \subseteq S
$$

The notation $X_{S}$ and $S^{X}$ wants to recall Ringel reflection functors [47] and we call $X_{S}\left(\right.$ resp. $\left.S^{X}\right)$ the Ringel reflection of $X$ at $S$ (resp. of $S$ at $X$ ). By definition, $X_{S}$ is the 
maximal subrepresentation of $X$ such that the push-out sequence $\pi_{*}(\xi)$ induced by the quotient $\pi: X \longrightarrow X / X_{S}$ does not split. Dually, $S^{X}$ is the minimal subrepresentation of $S$ such that the pull-back sequence $\iota^{*}(\xi)$ induced by the inclusion $\iota: S^{X} \rightarrow S$ does not split. In other words $X_{S}$ is the maximal subrepresentation of $X$ such that the quotient $X \longrightarrow X / X_{S}$ does not factor through $Y$. Dually, $S^{X}$ is the minimal subrepresentation of $S$ that does not factor through $Y$. In particular, if $X$ and $S$ are indecomposable such that $[S, X]^{1}=1$ and $\xi$ is almost split, then $X_{S}=0$ and $S^{X}=S$. The reverse implication is not true, and motivates the following definition.

Definition 29. A short exact sequence $0 \rightarrow X \rightarrow Y \rightarrow S \rightarrow 0$ is called generalized almost split if $[S, X]^{1}=1, X_{S}=0$ and $S^{X}=S$.

In other words a short exact sequence $0 \rightarrow X \rightarrow Y \rightarrow S \rightarrow 0$ is generalized almost split if $[S, X]^{1}=1$ and every proper quotient of $X$ and every proper subrepresentation of $S$ factors through $Y$. There are plenty of examples of generalized almost split sequences which are not almost split.

Example 30. For a quiver of type $A$, generalized almost split sequences are almost split, as one may check by direct inspection. For a quiver of type $D$ the situation is different. For example, let us consider the quiver of type $D_{4}$ with a unique sink in the central vertex 0 and let $i$ be a vertex different from 0 . Put $X=P_{0}$ and $S=\tau^{-} P_{i}$, then $[S, X]^{1}=1$ and a short exact sequence corresponding to a generator $\xi \in \operatorname{Ext}^{1}(S, X)$ is generalized almost split but not almost split.

It is easy to see that if $\xi$ is a generalized almost split sequence then $X$ and $S$ must be indecomposable.

Lemma 31. Let $X, S \in \operatorname{Rep}(\mathcal{Q})$ such that $[S, X]^{1}=1$.

(1) Let $f: X \rightarrow \tau S$ be a non-zero morphism; then $X_{S}=\operatorname{ker}(f)$.

(2) Let $g: \tau^{-} X \rightarrow S$ be a non-zero morphism; then $S^{X}=\operatorname{im}(g)$.

(3) The representations $X / X_{S}$ and $S^{X}$ are indecomposable and there exist exact sequences

$$
\begin{array}{r}
0 \rightarrow X / X_{S} \rightarrow \tau S^{X} \rightarrow I \rightarrow 0 \\
0 \rightarrow P \rightarrow \tau^{-}\left(X / X_{S}\right) \rightarrow S^{X} \rightarrow 0
\end{array}
$$

where $I$ is either injective or zero and $P=\nu^{-1}(I)$.

(4) The representations $X / X_{S}$ and $S^{X}$ are bricks.

(5) The vector space $\operatorname{Ext}^{1}\left(S^{X}, X / X_{S}\right)$ is generated by a generalized almost split sequence.

(6) Given $N_{1} \subseteq X$ and $N_{2} \subseteq S,\left[N_{2}, X / N_{1}\right]^{1}=0$ if and only if either $N_{1} \nsubseteq X_{S}$ or $N_{2} \nsupseteq S^{X}$. 
Proof. (1) We have $1=[S, X]^{1}=[X, \tau S]$ and let $f: X \rightarrow \tau S$ be a non-zero morphism. We show that $\operatorname{ker}(f)=X_{S}$. Let $p: X \longrightarrow Z$ be a proper quotient of $X$ such that $[S, Z]^{1}=1$. Then $[Z, \tau S]=1$ and there is a non-zero composite morphism $X \longrightarrow Z \longrightarrow \tau S$ which is hence a multiple of $f$. In particular, $\operatorname{ker}(p) \subseteq \operatorname{ker}(f)$. This shows that $X_{S} \subseteq \operatorname{ker}(f)$. On the other hand, $0 \neq \operatorname{im}(f) \subseteq \tau S$ and hence $0 \neq[\operatorname{im}(f), \tau S]=[S, \operatorname{im}(f)]^{1} \leq[S, X]^{1}=1$. It follows that $[S, \operatorname{im}(f)]^{1}=1$ and hence $\operatorname{ker}(f) \subseteq X_{S}$.

(2) We have $1=[S, X]^{1}=\left[\tau^{-} X, S\right]$, and hence there is a non-zero morphism $g: \tau^{-} X \rightarrow$ $S$. Let $L \subset S$ be a subrepresentation such that $[L, X]^{1}=1$; then $\left[\tau^{-} X, L\right]=1$ and there is a non-zero composite morphism $\tau^{-} X \rightarrow L \subset S$ which is hence a multiple of $g$. It follows that $\operatorname{im}(g) \subseteq L$ and hence $S^{X} \supseteq \operatorname{im}(g)$. On the other hand, $\tau^{-} X \longrightarrow \operatorname{im}(g)$ and hence $0 \neq\left[\tau^{-} X, \operatorname{im}(g)\right]=[\operatorname{im}(g), X]^{1} \leq[S, X]^{1}=1$. It follows that $[\operatorname{im}(g), X]^{1}=1$ and $\operatorname{im}(g) \supseteq S^{X}$.

(3) The representation $X / X_{S}$ is indecomposable by maximality of $X_{S}$ and $S^{X}$ is indecomposable by its minimality. Let us prove the existence of the sequences (11) and (12). Let $f: X \rightarrow \tau S$ be a non-zero morphism. Then, by part (1) $X / X_{S}$ is isomorphic to the image of $f$. Let $g=\tau^{-}(f): \tau^{-} X \rightarrow S$. By part (2) $S^{X}$ is the image of $g$. Since $\tau$ is left exact, $\tau S^{X} \subseteq \tau S$ and there exists a diagram

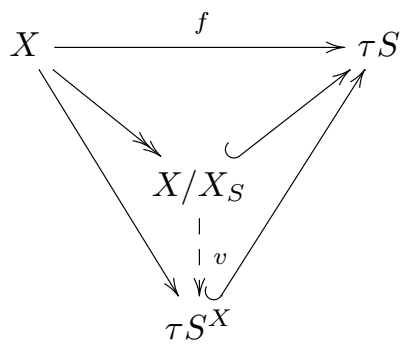

such that the two triangles with common side $\mathrm{f}$ commute (since $[X, \tau S]=1$ ). Since $X / X_{S}$ is the image of $f$ there exists a unique map $v: X / X_{S} \rightarrow \tau S^{X}$ which completes the diagram, and hence must be injective. Let $I=\operatorname{Coker}(v)$. We claim that $I$ is injective. Indeed, we apply $\tau^{-}$to the short exact sequence $0 \rightarrow X / X_{S} \rightarrow \tau S^{X} \rightarrow$ $I \rightarrow 0$ and we get the exact sequence $0 \rightarrow \nu^{-1} I \rightarrow \tau^{-}\left(X / X_{S}\right) \rightarrow S^{X} \rightarrow \tau^{-} I \rightarrow 0$. The morphism $\tau^{-}(v): \tau^{-}\left(X / X_{S}\right) \rightarrow S^{X}$ makes the following diagram commutative

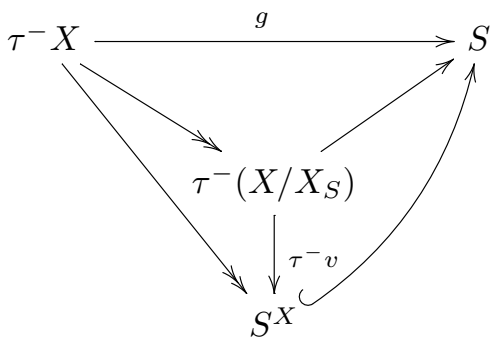


In particular, $\tau^{-} v$ is surjective and hence $\tau^{-} I=0$. It follows that $I$ is injective. The sequence (12) is obtained by applying $\tau^{-}$to (11).

(4) We apply $\operatorname{Hom}\left(S^{X},-\right)$ to $(11)$ and get a surjection

$$
\operatorname{Ext}^{1}\left(S^{X}, X / X_{S}\right) \longrightarrow \operatorname{Ext}^{1}\left(S^{X}, \tau S^{X}\right) .
$$

Since $\left[S^{X}, X / X_{S}\right]^{1} \leq 1$ and $\left[S^{X}, \tau S^{X}\right]^{1} \geq 1$ we see that $1=\left[S^{X}, X / X_{S}\right]^{1}=$ $\left[S^{X}, \tau S^{X}\right]^{1}$. This implies (4), since $\left[S^{X}, S^{X}\right]=\left[\tau S^{X}, \tau S^{X}\right]=\left[S^{X}, \tau S^{X}\right]^{1}=1$. To get that $X / X_{S}$ is a brick, apply $\operatorname{Hom}\left(-, X / X_{S}\right)$ to (12) and proceed similarly.

(5) We already proved that $1=\left[S^{X}, X / X_{S}\right]^{1}=\left[S^{X}, \tau S^{X}\right]^{1}$. Let $\eta \in \operatorname{Ext}^{1}\left(S^{X}, X / X_{S}\right)$ be non-split. Then $\eta$ has the form

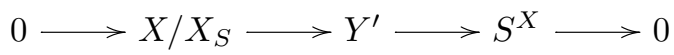

We need to show that every (proper) quotient of $X / X_{S}$ and every (proper) subrepresentation of $S^{X}$ factor through $Y^{\prime}$. This follows at once from the maximality of $X_{S}$ and the minimality of $S^{X}$ : indeed, let $X / X_{S} \longrightarrow Z$ be a proper quotient, then $[S, Z]^{1}=0$ and hence $\left[S^{X}, Z\right]^{1} \leq[S, Z]^{1}=0$; let $N \subsetneq S^{X}$, then $\left[N, X / X_{S}\right]^{1} \leq[N, X]^{1}=0$ by minimality of $S^{X}$.

(6) We need to prove that $\left[N_{2}, X / N_{1}\right]^{1}=0$ if and only if either $\left[N_{2}, X\right]^{1}=0$ or $\left[S, X / N_{1}\right]^{1}=0$. Since $\left[N_{2}, X\right]^{1} \geq\left[N_{2}, X / N_{1}\right]^{1}$ and $\left[S, X / N_{1}\right]^{1} \geq\left[N_{2}, X / N_{1}\right]^{1}$, one implication follows. To prove the other implication let us suppose, for a contradiction, that $\left[N_{2}, X / N_{1}\right]^{1}=0$ and both $\left[N_{2}, X\right]^{1}=\left[S, X / N_{1}\right]^{1}=1$. Then $N_{2} \supseteq S^{X}$ and $N_{1} \subseteq X_{S}$. It follows that $\left[N_{2}, X / N_{1}\right]^{1} \geq\left[S^{X}, X / N_{1}\right] \geq\left[S^{X}, X / X_{S}\right]^{1}=1$, a contradiction.

We can now give a more precise formulation of Theorem 26 for a non-split generating extension.

Theorem 32. Let $0 \neq \xi \in \operatorname{Ext}^{1}(S, X)$ be a generating extension. Then:

$$
\operatorname{Im}\left(\Psi_{\mathbf{f}, \mathbf{g}}^{\xi}\right)=\left(\operatorname{Gr}_{\mathbf{f}}(X) \times \operatorname{Gr}_{\mathbf{g}}(S)\right) \backslash\left(\operatorname{Gr}_{\mathbf{f}}\left(X_{S}\right) \times \operatorname{Gr}_{\mathbf{g}-\operatorname{dim} \mathbf{S}} \mathbf{x}\left(S / S^{X}\right)\right)
$$

and $\Psi_{\mathbf{f}, \mathbf{g}}^{\xi}: \mathcal{S}_{\mathbf{f}, \mathbf{g}}^{\xi} \rightarrow \operatorname{Im}\left(\Psi_{\mathbf{f}, \mathbf{g}}^{\xi}\right)$ is a Zariski-locally trivial affine bundle of rank $\langle\mathbf{g}, \operatorname{dim} X-$ f).

Proof. The description of the image follows from Lemma 31 (6). The rest is Theorem 26.

Corollary 33. Let $N$ be a non-projective brick and let $\xi: 0 \rightarrow \tau N \rightarrow E \rightarrow N \rightarrow 0$ be the almost split sequence ending in $N$. If $N$ and $\tau N$ have property $(C)$, then $E$ has property $(C)$. 
Proof. Since $N$ is a brick, $\xi$ is generating. Since $\xi$ is almost split, it is a generalized almost split sequence. The claim now follows from Theorem 32 .

The following corollary is useful for the application to cluster algebras given in Section 7 .

Corollary 34. Let $X$ and $S$ be two representations of $Q$ such that $[S, X]^{1}=1$. Let $\xi$ : $0 \rightarrow X \rightarrow Y \rightarrow S \rightarrow 0$ be a non-split short exact sequence. For any $\mathbf{f}+\mathbf{g}=\mathbf{e}$ there is a decomposition

$$
\operatorname{Gr}_{\mathbf{f}}(X) \times \operatorname{Gr}_{\mathbf{g}}(S)=\operatorname{Im}\left(\Psi_{\mathbf{f}, \mathbf{g}}^{\xi}\right) \coprod\left(\operatorname{Gr}_{\mathbf{f}}\left(X_{S}\right) \times \operatorname{Gr}_{\mathbf{g}-\operatorname{dim} \mathbf{S}} \mathbf{x}\left(S / S^{X}\right)\right)
$$

In particular the following relation between Euler characteristics holds

$$
\sum_{\mathbf{f}+\mathbf{g}=\mathbf{e}} \chi\left(\operatorname{Gr}_{\mathbf{f}}(X) \times \operatorname{Gr}_{\mathbf{g}}(S)\right)=\chi\left(\operatorname{Gr}_{\mathbf{e}}(Y)\right)+\chi\left(\operatorname{Gr}_{\mathbf{e}-\operatorname{dim} \mathbf{S}} \mathbf{x}\left(X_{S} \oplus S / S^{X}\right)\right)
$$

Proof. Since $[S, X]^{1}=1$ we have a dichotomy (see Lemma 31 (6) for the second equality)

$$
\begin{aligned}
\operatorname{Gr}_{\mathbf{f}}(X) \times \operatorname{Gr}_{\mathbf{g}}(S) & =\left\{\left(N_{1}, N_{2}\right) \mid\left[N_{2}, X / N_{1}\right]^{1}=0\right\} \coprod\left\{\left(N_{1}, N_{2}\right) \mid\left[N_{2}, X / N_{1}\right]^{1}=1\right\} \\
& =\operatorname{Im}\left(\Psi_{\mathbf{f}, \mathbf{g}}^{\xi}\right) \coprod\left(\operatorname{Gr}_{\mathbf{f}}\left(X_{S}\right) \times \operatorname{Gr}_{\mathbf{g}-\operatorname{dim} S^{X}}\left(S / S^{X}\right)\right) .
\end{aligned}
$$

Formula (13) then follows from Theorem 32.

\subsection{Description of Ringel reflections in particular cases}

In this section we investigate further $X_{S}$ and $S^{X}$ in particular cases.

Proposition 35. Let $X, S$ be exceptional $Q$-representations such that $[S, X]^{1}=1$ and $[X, S]^{1}=0$. Let $\xi: 0 \rightarrow X \rightarrow Y \rightarrow S \rightarrow 0 \in \operatorname{Ext}^{1}(S, X)$ be a generating extension. Then

(1) $S^{X}$ and $X / X_{S}$ are rigid bricks (even without the hypothesis $[X, S]^{1}=0$ ).

(2) $X \oplus Y \oplus X_{S} \oplus S / S^{X}$ is rigid.

(3) $S \oplus Y \oplus X_{S} \oplus S / S^{X}$ is rigid.

Proof. By Lemma 31(4) we know that $S^{X}$ and $X / X_{S}$ are bricks. To show that $S^{X}$ is rigid apply $\operatorname{Hom}(-, S)$ to the short exact sequence $0 \rightarrow \operatorname{Ker}(g) \rightarrow \tau^{-} X \rightarrow S^{X} \rightarrow 0$ (where $g$ is given by Lemma $31(2))$ to get $\left[S^{X}, S\right]=1$ and $[\operatorname{Ker}(g), S]=0$; then apply $\operatorname{Hom}\left(-, S^{X}\right)$ to the same sequence to get $\left[S^{X}, S^{X}\right]^{1} \leq\left[\operatorname{Ker}(g), S^{X}\right] \leq[\operatorname{Ker}(g), S]=0$. To show that $X / X_{S}$ is rigid, apply $\operatorname{Hom}(-, \tau S)$ to the short exact sequence $0 \rightarrow X_{S} \rightarrow$ $X \rightarrow X / X_{S} \rightarrow 0$ to get $\left[X_{S}, \tau S\right]=0$; then apply $\operatorname{Hom}(X,-)$ to the same sequence to get $\left[X / X_{S}, X / X_{S}\right]^{1} \leq\left[X_{S}, X / X_{S}\right] \leq\left[X_{S}, \tau S\right]=0$. 
The rigidity of the two modules in (2) and (3) is obtained by constructing long exact sequences of Ext - spaces. In each case it is straightforward to find such sequences and to obtain the claimed vanishing conditions.

Lemma 36. Let $X$ and $Y$ be indecomposable preprojective representations such that $[X, Y]=1,[X, \tau Y]=0$ and a non-zero morphism $\iota: X \rightarrow Y$ is mono. Let $S=\operatorname{Coker}(\iota)$. Then $[S, X]^{1}=1$ and hence $X_{S}$ and $S^{X}$ are well-defined. Let $E \rightarrow X$ be the minimal right almost split morphism ending in $X$. If $Y$ is not projective, then there is a decomposition $E=E_{0} \oplus E^{\prime}$ where $E_{0}$ is indecomposable such that $\left[S, E_{0}\right]^{1}=1$, $\left[S, E^{\prime}\right]^{1}=0, X_{S} \cong E^{\prime} \oplus \operatorname{Ker}\left(E_{0} \rightarrow \tau Y\right)$ and there exists a short exact sequence $0 \rightarrow X \rightarrow \operatorname{Im}\left(\tau^{-} E_{0} \rightarrow Y\right) \rightarrow S^{X} \rightarrow 0$. If $Y$ is projective then $X_{S}=E$.

Proof. From the proof of Unger's Lemma 8 we know that $[X, S]=0$. We apply $\operatorname{Hom}(-, X)$ to the short exact sequence $0 \rightarrow X \rightarrow Y \rightarrow S \rightarrow 0$ and get $\mathbf{C} \cong$ $(X, X) \rightarrow(S, X)^{1} \rightarrow(Y, X)^{1}=0$ and hence $[S, X]^{1}=1$. If $X$ is not-projective, let $0 \rightarrow \tau X \rightarrow E \rightarrow X \rightarrow 0$ be the almost split sequence ending in $X$. Since $[S, \tau X]^{1}=[\tau X, \tau S]=[X, S]=0$, it follows that $[S, E]^{1}=[S, X]^{1}=1$ and hence there exists a decomposition $E=E_{0} \oplus E^{\prime}$ with the stated properties. Since $X$ is not projective there is a commutative diagram with exact rows

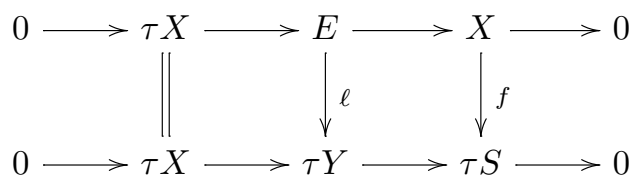

It follows that $X_{S}=\operatorname{ker}(f) \cong \operatorname{ker}(\ell)=E^{\prime} \oplus \operatorname{Ker}\left(E_{0} \rightarrow \tau Y\right)$, as claimed. If $X=P_{k}$ is projective, then $E=\operatorname{rad}(P)=\oplus_{k \rightarrow j} P_{j}$ and there is an exact sequence $0 \rightarrow E \rightarrow X \rightarrow$ $S_{k} \rightarrow 0$. If $Y$ is not projective, there exists a commutative diagram with exact rows

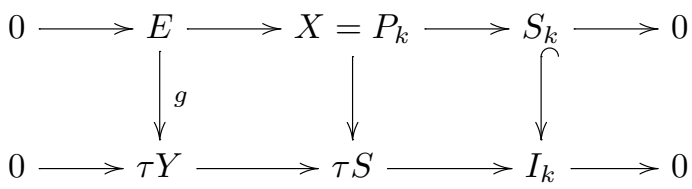

from which it follows that $X_{S} \cong \operatorname{ker}(g)$. Moreover $[E, \tau Y]=[E, \tau S]=1$ and hence $E$ admits the claimed decomposition. If $Y=P_{j}$ is projective (and $X=P_{k}$ is projective too), then $X_{S}=E$ since $X / E=S_{k}$. To conclude the proof, we consider the commutative diagram with exact rows

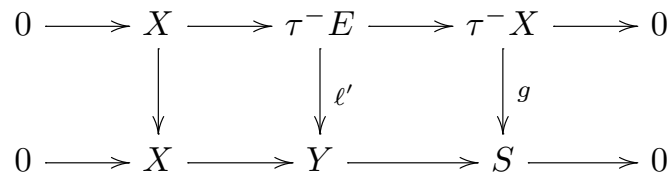


In particular $\operatorname{Coker}\left(\ell^{\prime}\right) \cong \operatorname{Coker}(g)$ and hence the claimed surjection

$$
\operatorname{im}\left(\ell^{\prime}\right) \longrightarrow \operatorname{im}(g)=S^{X}
$$

\section{Proof of Theorem 1}

In this section we prove the following theorem.

Theorem 37. Let $M$ be a rigid representation of a quiver and let $X=\operatorname{Gr}_{\mathbf{e}}(M)$ be a quiver Grassmannian attached to it. Then $X$ is irreducible, has property $(S)$ and the Chern classes of the universal bundles $\mathcal{U}_{i}$ on $X$ generate the Chow ring $A^{*}(X)$ as a ring.

We start by proving the irreducibility. The main idea of the proof was communicated to the authors by Julia Sauter.

Proposition 38. Any quiver Grassmannian $\operatorname{Gr}_{\mathbf{e}}(M)$ attached to a rigid quiver representation $M$ is irreducible.

Proof. We consider the universal quiver Grassmannian $\operatorname{Gr}_{\mathbf{e}}^{Q}(\mathbf{d}) \subseteq \operatorname{Gr}_{\mathbf{e}}(\mathbf{d}) \times \mathrm{R}_{\mathbf{d}}(Q)$ (see Section 2.3) and the map $p: \mathrm{Gr}_{\mathbf{e}}^{Q}(\mathbf{d}) \rightarrow \mathrm{R}_{\mathbf{d}}(Q)$ which projects to the second factor. Let $O(M)$ be the orbit of the representation $M$ by the group action of $G=\prod_{i} \mathrm{GL}_{d_{i}}(K)$ on $\mathrm{R}_{\mathbf{d}}(Q)$. The orbit is open in $\mathrm{R}_{\mathbf{d}}(Q)$ as $M$ is rigid. The inverse image $X=p^{-1}(O(M))$ is hence also open inside $\operatorname{Gr}_{\mathbf{e}}^{Q}(\mathbf{d})$ and thus, as $\operatorname{Gr}_{\mathbf{e}}^{Q}(\mathbf{d})$ is irreducible (it is an affine bundle over $\left.\operatorname{Gr}_{\mathbf{e}}(\mathbf{d})\right), X$ is also irreducible. As we work in characteristic zero, the orbit $O(M)$ is isomorphic to $G / \operatorname{Aut}(M)$ via the action map. The resulting morphism $f: X \rightarrow$ $G / \operatorname{Aut}(M)$ is $G$-equivariant and the fiber of $e \operatorname{Aut}(M)$ (the coset of the neutral element of $G$ ) is just $\operatorname{Gr}_{\mathbf{e}}(M)$. A general result yields that

$$
X \cong G \times{ }^{\operatorname{Aut}(M)} \operatorname{Gr}_{\mathbf{e}}(M)
$$

The lemma that we are using here is well-known and the proof is very easy; this might be the reason why we could not find a reference for it other than [27, Lem. 5.3]. The group $\operatorname{Aut}(M)$ is connected as it is open inside the vector space $\operatorname{End}(M)$. Now the claim follows from the following lemma.

Lemma 39. Let $G$ be a connected algebraic group, let $H \subseteq G$ be a connected closed subgroup of $G$, let $Y$ be an $H$-scheme and $X=G \times{ }^{H} Y$. Then $Y_{\alpha}$ is an irreducible component of $Y$ if and only if $X_{\alpha}=G \times{ }^{H} Y_{\alpha}$ is an irreducible component of $X$. In particular, $X$ is irreducible if and only if $Y$ is irreducible.

Proof. Since $H$ is connected, each irreducible component $Y_{\alpha}$ is $H$-stable and $X=\cup_{\alpha} X_{\alpha}$. The map $\varphi: G \times Y \rightarrow X$ is a principal $H$-bundle. Thus, $G \times Y_{\alpha}$ and hence $X_{\alpha}$ is 
irreducible. Since the map $\varphi$ is open, $X_{\alpha}$ is closed in $X$, and hence an irreducible component.

Remark 40. In fact Proposition 38 holds even without the hypothesis of characteristic zero. In positive characteristic the orbit is not necessarily isomorphic to $G / A u t(M)$, but there is a radicial homeomorphism $G / \operatorname{Aut}(M) \rightarrow \mathcal{O}(M)$ ([20, III.3.5.2]) and one applies Lemma 39 to the pullback of the map $X \rightarrow \mathcal{O}(M)$ to conclude.

A result of Ellingsrud and Strømme states:

Theorem 41 ([25, Th. 2.1]). Let $X$ be a smooth complete complex variety and assume that there exist $\alpha_{\lambda}, \beta_{\lambda} \in A^{*}(X)$ for all $\lambda$ in a finite set I such that the class of the diagonal $\Delta \subseteq X \times X$ decomposes as

$$
[\Delta]=\sum_{\lambda \in I} p_{1}^{*} \alpha_{\lambda} \cdot p_{2}^{*} \beta_{\lambda} \cap[X \times X]
$$

in $A_{*}(X \times X)$. Then the $\alpha_{\lambda}$ 's generate $A^{*}(X)$ as an abelian group and $X$ has property $(S)$.

In the above theorem, the maps $p_{1}, p_{2}: X \times X \rightarrow X$ are the projections to the first and second component, respectively. Note that $\left\{\beta_{\lambda} \mid \lambda \in I\right\}$ is also a set of generators for $A^{*}(X)$ as an abelian group.

Remark 42. We illustrate how we can use the decomposition of the diagonal to prove that the (usual) Grassmannian $\operatorname{Gr}_{k}(V)$ has property (S). Let $V$ be an $n$-dimensional $K$-vector space. Denote by $V_{X}$ the trivial vector bundle on $X=\operatorname{Gr}_{k}(V)$ with fiber $V$. Let $\mathcal{U} \subseteq V_{X}$ be the rank $k$-subbundle and let $\mathcal{Q}=V_{X} / \mathcal{U}$. On $X \times X$ we consider the composition of the morphisms

$$
p_{2}^{*} \mathcal{U} \rightarrow p_{2}^{*} V_{X}=V_{X \times X}=p_{1}^{*} V_{X} \rightarrow p_{1}^{*} \mathcal{Q}
$$

It gives a global section $s$ of the bundle $\underline{\operatorname{Hom}}\left(p_{2}^{*} \mathcal{U}, p_{1}^{*} \mathcal{Q}\right)=p_{2}^{*} \mathcal{U}^{\vee} \otimes p_{1}^{*} \mathcal{Q}$. The zero locus $Z(s)$ agrees - as a scheme - with the diagonal $\Delta \subseteq X \times X$. The codimension of $Z(s)$ inside $X \times X$ is $\operatorname{dim} X=k(n-k)$ which is the same as the rank of $p_{2}^{*} \mathcal{U} \otimes p_{1}^{*} \mathcal{Q}$. Therefore, [29, Prop. 14.1, Ex. 14.1.1] imply that

$$
[\Delta]=[Z(s)]=c_{k(n-k)}\left(p_{2}^{*} \mathcal{U}^{\vee} \otimes p_{1}^{*} \mathcal{Q}\right) \cap[X \times X]
$$

in $A_{*}(X \times X)$. If we denote by $\xi_{1}, \ldots, \xi_{k}$ the Chern roots of $\mathcal{U}^{\vee}$ then we obtain, using a result of Lascoux ([34], see also [29, Ex. 14.5.2]), the following expression for the top Chern class of the bundle $p_{2}^{*} \mathcal{U}^{\vee} \otimes p_{1}^{*} \mathcal{Q}$

$$
c_{k(n-k)}\left(p_{2}^{*} \mathcal{U}^{\vee} \otimes p_{1}^{*} \mathcal{Q}\right)=\sum_{\lambda} p_{2}^{*} s_{\lambda}\left(\xi_{1}, \ldots, \xi_{k}\right) \cdot p_{1}^{*} s_{\lambda^{c}}\left(\xi_{1}, \ldots, \xi_{k}\right) .
$$


The sum ranges over all partitions $\lambda$ with $n-k \geq \lambda_{1} \geq \ldots \geq \lambda_{k} \geq \lambda_{k+1}=0$ and $\lambda^{c}$ stands for the complementary partition inside the $k \times(n-k)$ box, i.e. $\lambda_{i}+\lambda_{k-i+1}^{c}=n-k$ for all $i$. As the brick functions $s_{\lambda}$ are symmetric functions, they can be expressed as polynomials in elementary symmetric functions. Hence $s_{\lambda}\left(\xi_{1}, \ldots, \xi_{k}\right)$ is a polynomial in the Chern classes of $\mathcal{U}^{\vee}$ (and these lie in $A^{*}(X)$ ). The requirements of Theorem 41 are fulfilled and thus $X$ has property $(\mathrm{S})$.

Proof of Theorem 37. Let $\mathbf{d}=\operatorname{dim} M$ be the dimension vector of the rigid representation $M$ and let $\mathbf{e} \leq \mathbf{d}$ be a sub-dimension vector. We consider $X=\operatorname{Gr}_{\mathbf{e}}(M)$. It is a smooth projective variety of dimension $\langle\mathbf{e}, \mathbf{d}-\mathbf{e}\rangle$. Let $\mathcal{U}_{i}$ be the pull-back of the universal subbundle along $\operatorname{Gr}_{\mathbf{e}}(M) \rightarrow \prod_{j \in Q_{0}} \operatorname{Gr}_{\mathbf{e}_{j}}\left(M_{j}\right) \rightarrow \operatorname{Gr}_{\mathbf{e}_{i}}\left(M_{i}\right)$ and let $\mathcal{Q}_{i}$ be the pull-back of the universal quotient bundle along the same morphism. We consider the split short exact sequence

$$
\eta: 0 \rightarrow M \stackrel{\iota_{1}}{\rightarrow} M \oplus M \stackrel{\pi_{2}}{\longrightarrow} M \rightarrow 0
$$

On $X \times X$ we consider the vector bundles

$$
H:=\bigoplus_{i} \underline{\operatorname{Hom}}\left(p_{2}^{*} \mathcal{U}_{i}, p_{1}^{*} \mathcal{Q}_{i}\right), \quad K:=\bigoplus_{\alpha} \underline{\operatorname{Hom}}\left(p_{2}^{*} \mathcal{U}_{s(\alpha)}, p_{1}^{*} \mathcal{Q}_{t(\alpha)}\right)
$$

(as defined in Equations (7) and (8)) and the homomorphism $\Phi: H \rightarrow K$ of vector bundles as defined in Equation (9). Since $M$ is rigid, the map $\Phi$ is surjective and hence $\operatorname{ker}(\Phi)=\underline{\operatorname{Hom}}_{Q}\left(p_{2}^{*} \mathcal{U}, p_{1}^{*} \mathcal{Q}\right)$ is a vector bundle of rank $\langle\mathbf{e}, \mathbf{d}-\mathbf{e}\rangle$. Let us consider the global section $s$ of $H$ given by

$$
p_{2}^{*} \mathcal{U}_{i} \rightarrow p_{2}^{*}\left(M_{i}\right)_{X}=\left(M_{i}\right)_{X \times X}=p_{1}^{*}\left(M_{i}\right)_{X} \rightarrow p_{1}^{*} \mathcal{Q}_{i}
$$

This section is in fact a global section of the subbundle $\operatorname{ker}(\Phi)$ : the fiber of the bundle $\underline{\operatorname{Hom}}_{Q}\left(p_{2}^{*} \mathcal{U}, p_{1}^{*} \mathcal{Q}\right)$ in a point $\left(U^{\prime}, U^{\prime \prime}\right)$ is $\operatorname{Hom}_{Q}\left(U^{\prime \prime}, M / U^{\prime}\right)$ and the composition $U^{\prime \prime} \rightarrow$ $M \rightarrow M / U^{\prime}$ is a morphism of $K Q$-modules. The zero locus $Z(s)$ coincides with the diagonal $\Delta$ as a subscheme of $X \times X$. Then properties of the localized top Chern class [29, Prop. 14.1, Ex. 14.1.1] imply

$$
[\Delta]=[Z(s)]=i_{\Delta, *} \mathbb{Z}(s)=c_{\langle e, d-e\rangle}\left(\underline{\operatorname{Hom}}_{Q}\left(p_{2}^{*} \mathcal{U}, p_{1}^{*} \mathcal{Q}\right)\right) \cap[X \times X]
$$

because $X$ is smooth and the zero locus has the correct dimension. This Chern class agrees with the $\langle\mathbf{e}, \mathbf{d}-\mathbf{e}\rangle$ th coefficient of the power series $c_{t}(\mathcal{E}) \cdot c_{t}(\mathcal{F})^{-1}$. Let $\xi_{i, 1}, \ldots, \xi_{i, \mathbf{e}_{i}}$ be the Chern roots of $\mathcal{U}_{i}^{\vee}$. By Lascoux's result, the Chern polynomial of the bundle $\mathcal{E}=\bigoplus_{i} \underline{\operatorname{Hom}}\left(p_{2}^{*} \mathcal{U}_{i}, p_{1}^{*} \mathcal{Q}_{i}\right)$ computes as

$$
c_{t}(\mathcal{E})=\prod_{i} \sum_{\lambda, \mu} \mathbf{d}_{\lambda, \mu} \cdot p_{2}^{*} s_{\mu}\left(\xi_{i, 1}, \ldots, \xi_{i, \mathbf{e}_{i}}\right) \cdot p_{1}^{*} s_{\lambda^{c}}\left(\xi_{i, 1}, \ldots, \xi_{i, \mathbf{e}_{i}}\right) \cdot t^{\mathbf{e}_{i}\left(\mathbf{d}_{i}-\mathbf{e}_{i}\right)+|\mu|-|\lambda|}
$$


In the above equation the sum ranges over all partitions $\lambda$ and $\mu$ where $\mathbf{d}_{i}-\mathbf{e}_{i} \geq \lambda_{1} \geq$ $\ldots \geq \lambda_{\mathbf{e}_{i}} \geq \lambda_{\mathbf{e}_{i}+1}=0$ and $\mu \subseteq \lambda$. The coefficient $\mathbf{d}_{\lambda, \mu}$ is the determinant of the $\left(\mathbf{e}_{i} \times \mathbf{e}_{i}\right)$ matrix whose $(k, l)$ th entry is $\left(\begin{array}{c}\lambda_{k}+\mathbf{e}_{i}-k \\ \mu_{l}+\mathbf{e}_{i}-l\end{array}\right)$. The formula for the Chern polynomial of $\mathcal{F}$ is similar. Let $\delta(X) \subseteq A^{*}(X \times X)$ be the subset of all $\gamma$ which possess a decomposition

$$
\gamma=\sum_{\lambda} p_{1}^{*} \alpha_{\lambda} \cdot p_{2}^{*} \beta_{\lambda}
$$

for some classes $\alpha_{\lambda}, \beta_{\lambda} \in A^{*}(X)$. It is easy to see that $\delta(X)$ is a subring of $A^{*}(X \times X)$. We have argued that $c_{t}(\mathcal{E})$ and $c_{t}(\mathcal{F})$ lie in $\delta(X)[t]$. As the constant coefficient of $c_{t}(\mathcal{F})$ is one, also its inverse is a power series whose coefficients lie in $\delta(X)$. This shows that all coefficients of $c_{t}(\mathcal{E}) \cdot c_{t}(\mathcal{F})^{-1}$ lie in $\delta(X)$; in particular the $\langle\mathbf{e}, \mathbf{d}-\mathbf{e}\rangle$ th. We are hence in the situation of Ellingsrud-Strømme's theorem, proving Theorem 37.

Corollary 43. The map $\iota^{*}: \mathrm{H}^{\bullet}\left(\prod_{i \in Q_{0}} \operatorname{Gr}_{\mathbf{e}_{i}}\left(M_{i}\right)\right) \rightarrow \mathrm{H}^{\bullet}\left(\operatorname{Gr}_{\mathbf{e}}(M)\right)$ (induced by the closed embedding $\left.\iota: \operatorname{Gr}_{\mathbf{e}}(M) \rightarrow \prod_{i} \operatorname{Gr}_{\mathbf{e}_{i}}\left(M_{i}\right)\right)$ is surjective.

Proof. By Theorem 37, $\mathrm{H}^{\bullet}\left(\mathrm{Gr}_{\mathbf{e}}(M)\right)$ is generated by algebraic cycles which are restrictions of universal bundles on the product of Grassmannians.

Corollary 44. Every $Q$-representation $M$ whose regular part $M_{\mathcal{R}}$ is rigid, has property $(S)$.

Proof. Let $M=M(1) \oplus \cdots \oplus M(N)$ be the decomposition of $M$ as direct sum of its indecomposable direct summands. Let $M_{\mathcal{P}}=M(1) \oplus \cdots \oplus M(p), M_{\mathcal{R}}=M(p+$ 1) $\oplus \cdots \oplus M(r)$ and $M_{\mathcal{I}}=M(r+1) \oplus \cdots \oplus M(N)$ be the preprojective, regular and preinjective part of $M$, respectively (see section 2.1). By assumption, $M_{\mathcal{R}}$ is rigid. Thus, every indecomposable direct summand of $M$ is rigid and hence, by Theorem 37, it has property $(\mathrm{S})$. Since the categories of preprojectives and preinjectives are directed, in view of (1), we can reorder the indecomposable direct summands of $M$ so that $[M(i), M(j)]^{1}=$ 0 for every $i \leq j$. We can hence apply repeatedly the split version of Theorem 26 and Lemma 13 to get the result.

\subsection{Proof of Corollary 2}

Let $M$ be a rigid representation of a quiver $Q$ over an algebraically closed field $K$, let $\mathbf{d}=\operatorname{dim} M$ be its dimension vector and let $\operatorname{Gr}_{\mathbf{e}}(M)$ be a non-empty quiver Grassmannian attached to it. There exists a unique (up to isomorphism) rigid $\mathbb{Z}$-form $M_{\mathbb{Z}} \in \mathbb{Z} Q-\bmod$ such that $M \cong M_{\mathbb{Z}} \otimes K$, and $M_{F}=M_{\mathbb{Z}} \otimes F$ is a rigid $F Q$-module for any field $F[16]$. The $\mathbb{Z}$-form $M_{\mathbb{Z}}$ defines a scheme $\mathcal{X} \rightarrow \operatorname{Spec}(\mathbb{Z})$ over $\mathbb{Z}$ whose geometric fibers are $\operatorname{Gr}_{\mathbf{e}}\left(M_{F}\right)$. The scheme $\mathcal{X}$ is a subscheme of the product $\mathcal{X}_{0}=\prod_{i} \mathcal{G} r_{\mathbb{Z}}\left(\mathbf{e}_{i}, \mathbf{d}_{i}\right)$ of Grassmannians over $\mathbb{Z}$ (see Section 2.3). 
Lemma 45. The scheme $\mathcal{X}$ is a projective, smooth, absolutely irreducible, complete intersection subscheme of $\mathcal{X}_{0}$ of dimension $\langle\mathbf{e}, \mathbf{d}-\mathbf{e}\rangle+1$.

Proof. By definition $\mathcal{X}$ is a closed subscheme of $\mathcal{X}_{0}$ and hence projective. Thus the map $p: \mathcal{X} \rightarrow \operatorname{Spec}(\mathbb{Z})$ is proper [39, Th. II.7.2]. Let $K$ be an algebraically closed field, since $M_{K}$ is rigid, the base change $\mathcal{X}_{K}$ of $\mathcal{X}$ to $K$ is smooth of dimension $\langle\mathbf{e}, \mathbf{d}-\mathbf{e}\rangle$, and irreducible by Proposition 38 and Remark 40. The same proof as [12, Prop. 3.1 (iii)] shows that $\mathcal{X}$ is complete intersection with $\sum_{i \rightarrow j \in Q_{1}} \mathbf{e}_{i}\left(\mathbf{d}_{j}-\mathbf{e}_{j}\right)$ number of equations inside the scheme $\mathcal{X}_{0}$ of dimension $\sum_{i \in Q_{0}} \mathbf{e}_{i}\left(\mathbf{d}_{i}-\mathbf{e}_{i}\right)+1$. So, $\langle\mathbf{e}, \mathbf{d}-\mathbf{e}\rangle+1$ is the dimension of the irreducible components of $\mathcal{X}$ which must all dominate $\operatorname{Spec}(\mathbb{Z})$. Thus $\mathcal{X}$ is irreducible, since the generic fiber is irreducible. Hence the morphism $p: \mathcal{X} \rightarrow \operatorname{Spec}(\mathbb{Z})$ is equidimensional in the sense of $[31,13.2 .2]$. By $[31,14.4 .4,15.2 .2], p$ is flat and hence smooth.

For a prime $p$, let us denote by $K=\mathbb{Q}$ and by $\kappa=\mathbb{F}_{p}$. Then there is a commutative diagram:

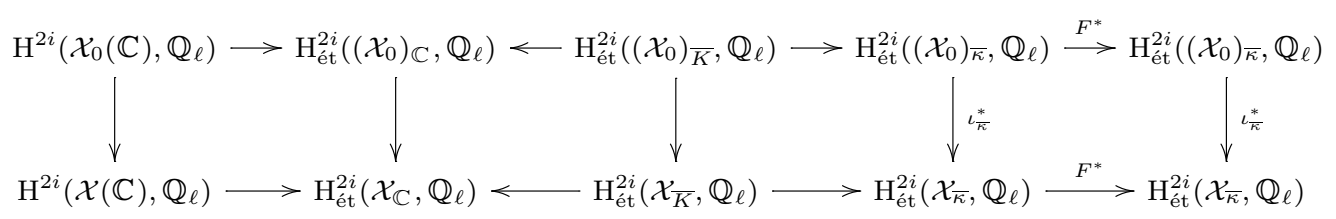

where: 1) the horizontal arrows are isomorphisms (this follows from [19, Th. 6.3 pg. 51, Cor. 3.3 pg. 63 , Th. 3.1 pg. 62 ], passing to the limit, applied to $\mathcal{X}$ and $\mathcal{X}_{0}$ which are proper smooth over $\mathbb{Z}_{(p)}$, the localization of $\mathbb{Z}$ at $\left.\left.p\right) ; 2\right) F^{*}$ is induced by the geometric Frobenius morphism; 3) the leftmost vertical arrow is surjective by Corollary 43 . It follows that $\iota_{\bar{\kappa}}^{*}$ is surjective. Moreover, since $F^{*}$ acts as multiplication by $p^{i}$ on $\mathrm{H}_{\text {ét }}^{2 i}\left(\left(\mathcal{X}_{0}\right)_{\bar{\kappa}}, \mathbb{Q}_{\ell}\right)\left(\mathcal{X}_{0}\right.$ is a product of Grassmannians), then $F^{*}$ acts by the same scalar on $\mathrm{H}_{\text {ét }}^{2 i}\left(\mathcal{X}_{\bar{\kappa}}, \mathbb{Q}_{\ell}\right)$. By the Grothendieck-Lefschetz trace formula (see e.g. [18, (1.5.1)]):

$$
\left|\mathcal{X}\left(\mathbb{F}_{p^{n}}\right)\right|=\sum_{i}(-1)^{i} \operatorname{Tr}\left(\left(F^{*}\right)^{n}, \mathrm{H}_{\text {ét }}^{i}\left(\mathcal{X}_{\bar{\kappa}}, \mathbb{Q}_{\ell}\right)\right),
$$

calling $b_{i}=\operatorname{dim}_{\mathbb{Q}} H^{i}(\mathcal{X}(\mathbb{C}), \mathbb{Q})=\operatorname{dim}_{\mathbb{Q}_{\ell}} \mathrm{H}^{i}\left(\mathcal{X}(\mathbb{C}), \mathbb{Q}_{\ell}\right)$ we get, for any power of a prime $q=p^{n}$

$$
\left|\mathcal{X}\left(\mathbb{F}_{q}\right)\right|=\sum_{i} b_{2 i} q^{i}=P_{\mathcal{X}}(q) \in \mathbb{Z}_{\geq 0}[q]
$$

\section{Proof of Theorem 4: the Dynkin case}

Theorem 46. Every representation of a Dynkin quiver has property $(C)$. 
Proof. Let $Q$ be a Dynkin quiver and let $M$ be a $Q$-representation. We prove that every (non-empty) quiver Grassmannian $\operatorname{Gr}_{\mathbf{e}}(M)$ attached to $M$ admits a cellular decomposition. The indecomposables $M(1), \cdots, M(N)$ of $\operatorname{Rep}_{K}(Q)$ can be ordered so that $\operatorname{Ext}^{1}(M(i), M(j))=0$ if $i>j$. Then, by Theorem 26, it is enough to prove Theorem 46 for $M$ indecomposable. We proceed by induction on the number of vertices of $Q$. The base of the induction being clear, we hence suppose the statement true for any subquiver of $Q$. In particular we may assume that $M$ is sincere, i.e. $\operatorname{supp}(M)=Q$. Let $\mathbf{d}=\operatorname{dim} M$. Suppose, first, that $\mathbf{d}$ is not the longest root of $E_{8}$. Then $\mathbf{d}$ is minuscule, i.e. there exists a leaf $i \in Q_{0}$ such that $\mathbf{d}_{i}=1$. We may assume that $i$ is a sink. For such $i$, either $\mathbf{e}_{i}=0$ or $\mathbf{e}_{i}=1$. Let $L \subseteq M$ be the subrepresentation generated by $M_{i}$ (in fact it is $P_{i}$ ) and let $\bar{M} \subseteq M$ be such that $M / \bar{M}=S_{i}$. In both cases one sees that $\operatorname{Gr}_{\mathbf{e}}(\bar{M})$ is isomorphic to a quiver Grassmannian for a representation supported in a smaller quiver: namely if $\mathbf{e}_{i}=0$ then $\operatorname{Gr}_{\mathbf{e}}(\bar{M}) \stackrel{\sim}{\rightarrow} \operatorname{Gr}_{\mathbf{e}}(M)$ and if $\mathbf{e}_{i}=1$ then $\operatorname{Gr}_{\mathbf{e}-\operatorname{dim} L}(M / L) \stackrel{\sim}{\rightarrow} \operatorname{Gr}_{\mathbf{e}}(M)$ (to see this, notice that those natural maps are bijective regular morphisms of algebraic varieties inducing isomorphisms on tangent spaces; since $M$ is rigid, $\operatorname{Gr}_{\mathbf{e}}(M)$ is smooth and hence they are isomorphisms). By induction we get the claim. If $\mathbf{d}$ is the longest root of $E_{8}$, then Lemma 47 below and Corollary 33 ends the proof.

Lemma 47. Let $M$ be an indecomposable of dimension vector equal to the longest root of type $E_{8}$, then it is the middle term of an almost split sequence.

Proof. Let $Q$ be an arbitrary orientation of the Dynkin diagram of type $E_{8}$ given by

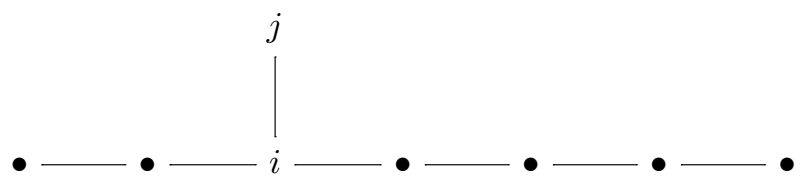

and let $\mathbf{d}=\operatorname{dim} M$ be the longest root:

For an indecomposable $U$, consider the starting function $s_{U}$ which associates to any representation $V$ the number $\operatorname{dim} \operatorname{Hom}_{Q}(U, V)$. The starting function $s_{P_{i}}$ assumes the value 6 on $M$, namely $s_{P_{i}}(M)=\operatorname{dim} \operatorname{Hom}_{Q}\left(P_{i}, M\right)=\operatorname{dim} M_{i}=6$. All starting functions for (extended) Dynkin quivers are listed in Bongartz's paper [4]. Now by direct inspection we see that the value 6 is only assumed in the $\tau$-orbit of $P_{i}$, thus M lies in that orbit. Consider the AR sequence ending in $M$, which has three middle terms. Precisely one of these middle terms, say $N$, belongs to the $\tau$-orbit of $P_{j}$. Thus the AR sequence ending in $N$ necessarily is of the form $0 \rightarrow N \rightarrow M \rightarrow \tau^{-} N \rightarrow 0$. 
Table 1

Affine diagrams and the minimal positive imaginary roots.

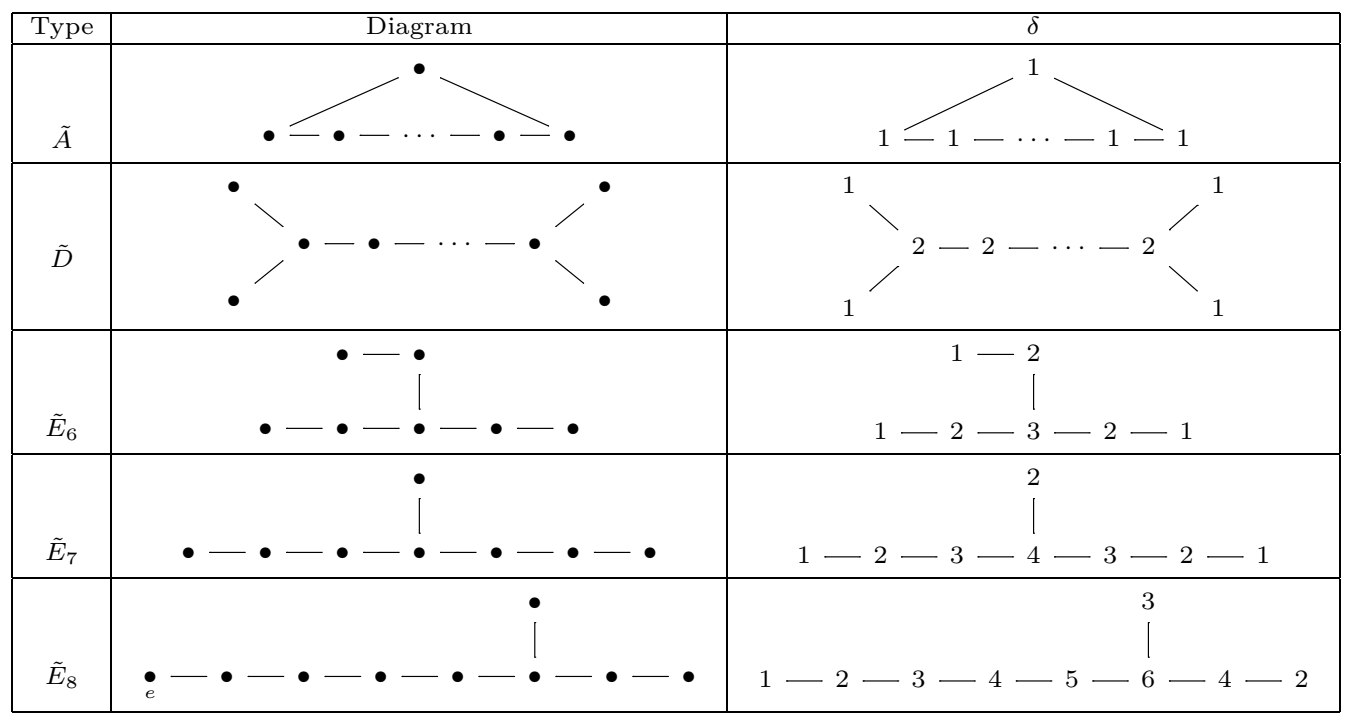

\section{Proof of Theorem 4: the affine case}

In this section we prove that every indecomposable representation of an affine quiver $Q$ has property (C), in the sense that every quiver Grassmannian attached to it admits a cellular decomposition. Clearly, it is enough to deal with connected quivers. Thus, throughout the section $Q$ denotes an acyclic orientation of one of the extended Dynkin diagrams of type $\tilde{A}_{n}, \tilde{D}_{n}, \tilde{E}_{6}, \tilde{E}_{7}$ and $\tilde{E}_{8}$ depicted in Table 1 . In Section 6.1 we prove the result for the regular modules and in Section 6.2 we prove the result for the preprojective modules. By duality we hence get the result for the preinjectives.

\subsection{Regular case}

Let $Q$ be an affine quiver shown in Table 1. Let us recall some well-known facts about regular modules over affine quivers (see e.g. [15] or [53]). We denote by $\partial_{Q}: \mathbb{Z}^{Q_{0}} \rightarrow \mathbb{Z}$ the defect of $Q$ which is the linear form given by $\partial_{Q}(\mathbf{x}):=\langle\delta, \mathbf{x}\rangle$. An indecomposable $Q$-representation $M$ is preprojective, regular or preinjective if and only if its defect $\partial_{Q}(M)$ is less, equal or greater than zero, respectively. By using the defect, it is easy to see that the category $\mathcal{R}$ of regular $Q$-representations is an abelian category. This fact is not true for wild quivers (see e.g. [15]). The simple objects of $\mathcal{R}$ are precisely the quasi-simple regular $Q$-representations. Every indecomposable regular $Q$-representation $R$ admits a unique filtration:

$$
R_{0}=0 \subset R_{1} \subset R_{2} \subset \cdots \subset R_{n-1} \subset R_{n-1} \subset R_{n}=R
$$


such that each $R_{i}$ is indecomposable regular and the successive quotients $S_{k}:=R_{k} / R_{k-1}$ are regular quasi-simple modules. Moreover the inclusions $R_{k-1} \subset R_{k}$ are irreducible. The quasi-simples $S_{1}=R_{1}, S_{2}, \cdots, S_{n}$ are called the regular composition factors of $R$; $R_{1}=S_{1}$ is called the regular socle of $R$ and $S_{n}$ is called the regular top of $R$. They satisfy the property $S_{k-1} \cong \tau S_{k}$ for every $k=2, \cdots, n$. The number $n$ is called the regular length of $R$, and $R_{n-1}$ is called the regular radical of $R$. Every indecomposable $R$ is uniquely determined by its regular-length and its top $S$. For an indecomposable regular $R$ with regular-length $n$ and regular top $S$ we sometimes use the notations $R=R_{n}=R_{n}(S)$ and we denote by $R_{i}=R_{i}(S)$ the regular subrepresentation of regularlength $i$. It is worth noting that quasi-simple (indecomposable) regular representations do not have proper regular subrepresentations (since they are regular simples). In particular they are bricks. The restriction of the two endofunctors $\tau$ and $\tau^{-}$to $\mathcal{R}$ define two inverse equivalences. The $\tau$-orbit of each indecomposable is finite and the order depends only on the regular top. The regular components of the AR-quiver are hence standard tubes. There are infinitely many tubes of rank one and at most three tubes of rank $>1$ which are called exceptional. The ranks of the exceptional tubes can be found in [23].

Proposition 48. Every quasi-simple regular $Q$-representation $S$ has property $(C)$. Moreover, if $S$ and $T$ are regular quasi-simple such that $\operatorname{dim} S=\operatorname{dim} T=\delta$, then $\operatorname{Gr}_{\mathbf{e}}(S) \cong \operatorname{Gr}_{\mathbf{e}}(T)$ for every $\mathbf{e}$.

Proof. Let $\delta$ be the minimal positive imaginary root of $q_{Q}$ and let $\mathbf{d}=\operatorname{dim} S$. Let $i \in Q_{0}$ be an extending vertex of $Q$, i.e. a vertex such that $\delta_{i}=1$ (see Table 1 ). Up to duality, we can assume that $i$ is a source of $Q$. We show that a non-empty quiver Grassmannian $\operatorname{Gr}_{\mathbf{e}}(S)$ admits a cell decomposition. If $S$ is not supported on the whole quiver $Q$ then $S$ is supported on a Dynkin quiver and hence it has property (C) by Theorem 46 . We hence assume that $S$ is sincere. It is known (see e.g. [14, Sec. 9, Lem. 3]) that $\mathbf{d} \leq \delta$. It follows that $\mathbf{d}_{i}=1$. For every subrepresentation $N \subseteq S$ we have $[N, S / N]^{1}=0$ and hence $\operatorname{Gr}_{\mathbf{e}}(S)$ is smooth. We can now argue as in the proof of Theorem 46: let $L(S) \subseteq S$ be the subrepresentation generated by $S_{i}$ and let $\bar{S} \subset S$ be such that $S / \bar{S}=S_{i}$. If $\mathbf{e}_{i}=0$ then $\operatorname{Gr}_{\mathbf{e}}(\bar{S}) \stackrel{\sim}{\rightarrow} \operatorname{Gr}_{\mathbf{e}}(S)$ and if $\mathbf{e}_{i}=1$ then $\operatorname{Gr}_{\mathbf{e}-\operatorname{dim} L(S)}(S / L(S)) \stackrel{\sim}{\rightarrow} \operatorname{Gr}_{\mathbf{e}}(S)$ and the claim follows by Theorem 46 . To prove the last statement we notice that $\bar{S} \cong \bar{T}$ and $S / L(S) \cong T / L(T)$, since they are rigid of the same dimension.

Proposition 49. Every indecomposable regular $Q$-representation has property $(C)$.

Proof. Let $R=R_{n}(S)$ be an indecomposable regular representation of regular-length $n$ and regular-top $S$. If $n=1$ then $R=S$ is quasi-simple, and the result is proved in Proposition 48. Suppose $n \geq 2$. Then there is a short exact sequence

$$
\xi_{n}: 0 \longrightarrow R_{n-1} \stackrel{\iota}{\longrightarrow} R_{n} \stackrel{\pi}{\longrightarrow} S \longrightarrow 0
$$


We notice that $\left[S, R_{n-1}\right]^{1}=\left[R_{n-1}, \tau S\right]=1$ since $\tau S \cong R_{n-1} / R_{n-2}$ is the regular-top of $R_{n-1}$ and the quasi-simple regular representations are bricks. We have (see Definition 28)

$$
(S)^{R_{n-1}}=S, \quad\left(R_{n-1}\right)_{S}=R_{n-2}
$$

(This follows directly from Lemma 31 parts 1 and 2.) We claim that each stratum $\mathcal{S}_{\mathbf{f}, \mathbf{g}}^{\xi_{n}}$ admits a cellular decomposition for every $n \geq 2$. We proceed by induction on $n \geq 2$. If $n=2$, then $\xi_{2}$ is almost split: by Theorem 32 we have an affine bundle $\mathcal{S}_{\mathbf{f}, \mathbf{g}}^{\xi_{2}} \longrightarrow \operatorname{Gr}_{\mathbf{f}}(\tau S) \times \operatorname{Gr}_{\mathbf{g}}(S)$ and we are done. Suppose $n \geq 3$ : we apply Theorem 32 to the exact sequence $\xi_{n}$ and in view of (15) we get affine bundles

$$
\mathcal{S}_{\mathbf{f}, \mathbf{g}}^{\xi_{n}} \longrightarrow \operatorname{Gr}_{\mathbf{f}}\left(R_{n-1}\right) \times \operatorname{Gr}_{\mathbf{g}}(S) \text { for } \mathbf{g} \neq \operatorname{dim} S, \quad \mathcal{S}_{\mathbf{f}, \operatorname{dim} S}^{\xi_{n}} \longrightarrow \mathcal{U}_{\mathbf{f}}\left(R_{n-1}, R_{n-2}\right)
$$

where $\mathcal{U}_{\mathbf{f}}\left(R_{n-1}, R_{n-2}\right):=\operatorname{Gr}_{\mathbf{f}}\left(R_{n-1}\right) \backslash \operatorname{Gr}_{\mathbf{f}}\left(R_{n-2}\right)$. By definition,

$$
\mathcal{U}_{\mathbf{f}}\left(R_{n-1}, R_{n-2}\right)=\coprod_{\mathbf{f}^{\prime}+\mathbf{f}^{\prime \prime}=\mathbf{f}, \mathbf{f}^{\prime \prime} \neq \mathbf{0}} \mathcal{S}_{\mathbf{f}^{\prime}, \mathbf{f}^{\prime \prime}}^{\xi_{n-1}}
$$

The claim follows by induction.

Remark 50. Formulas (15) can be deduced from [46, Sec. 4]. Indeed Ringel proved the following: let $p: R_{n-1} \longrightarrow Z$ be an indecomposable quotient of $R_{n-1}$. Ringel noticed that if $Z ¥ R_{n-1} / R_{i}$ for $i=0, \cdots, n-2$, then $p$ factors through $R_{n}$. This is another way to say that $\left(R_{n-1}\right)_{S}=R_{n-2}$. Actually Ringel showed more: he proved that any morphism $R_{n-1} \rightarrow Z$ (not necessarily epi) factors through $R_{n}$ on the above hypothesis. He called this property "extensions of homomorphisms".

\subsection{Preprojective case}

Let $\mathcal{P}(Q)$ denote the subcategory of preprojective $Q$-representations. In this section we prove that every object of $\mathcal{P}(Q)$ has property $(\mathrm{C})$.

An indecomposable $M \in \mathcal{P}(Q)$ has the form $M=\tau^{-k} P_{i}$ and it has defect $\partial(M):=$ $\langle\delta, \operatorname{dim} M\rangle=-\delta_{i}$. The following well-known fact will be used several times.

Lemma 51. Let $M$ and $N$ be indecomposable preprojective representations of an affine quiver.

(1) If $|\partial(M)| \leq|\partial(N)|$ and $[N, M]^{1}=0$ then any non-zero morphism $f: M \rightarrow N$ is mono.

(2) If there exists an epimorphism $g: M \longrightarrow N$ then $|\partial(M)|>|\partial(N)|$. 
Proof. By Happel-Ringel Lemma $7, f$ is either mono or epi. If $f$ is epi, its kernel has positive defect and hence it cannot be preprojective. We conclude that $f$ must be injective. To prove (2) we notice that the defect of the kernel of $g$, being preprojective, has negative defect.

\subsubsection{Type $\tilde{A}_{n}, \tilde{D}_{n}, \tilde{E}_{6}, \tilde{E}_{7}$}

Let $Q$ be an acylic orientation of an extended Dynkin diagram of one of the following types: $\tilde{A}_{n}, \tilde{D}_{n}, \tilde{E}_{6}, \tilde{E}_{7}$. In this section we prove that every preprojective and every preinjective representation of $Q$ has property (C). In order to deal with all cases at once, we introduce the notion of a "good monomorphism". Recall that given two indecomposable preprojective $Q$-representations $X$ and $Y$, a sectional morphism $f: X \rightarrow Y$ is the composition

$$
f: X=X_{0} \stackrel{f_{1}}{\longrightarrow} X_{1} \stackrel{f_{2}}{\longrightarrow} X_{2} \longrightarrow \cdots \longrightarrow X_{t-1} \stackrel{f_{t}}{\longrightarrow} X_{t}=Y
$$

of irreducible morphisms $f_{i}$ 's such that $X_{i-2} ¥ \tau X_{i}$; in this case we say that $f$ factors through the modules $X_{i}$. We notice that $f$ is either mono or epi (in type $\tilde{A}$ it is mono, by Lemma 51, in the other affine types this follows by Happel-Ringel Lemma 7). We say that $f$ is a minimal sectional mono if it is mono and each morphism $X_{i} \rightarrow Y$ is epi for $i=1,2, \cdots, t$. In type $\tilde{A}$ a minimal sectional mono is irreducible by Lemma 51 . An indecomposable preprojective $X=\tau^{-k} P_{i}$ is called a branch module if $i \in Q_{0}$ is a branch vertex, i.e. it is connected to three vertices.

Definition 52. Let $X$ and $Y$ be indecomposable preprojective $Q$-representations. A monomorphism $f: X \hookrightarrow Y$ is called a good mono if $f$ is a sectional morphism which factors through at most one branch module. A good mono is called minimal good mono if it is both a good mono and a minimal sectional mono.

Example 53. Let $Q$ be a quiver of type $\tilde{D}_{n}$ and let $Y$ be an indecomposable preprojective $Q$-representation of defect $(-1)$. The section ending in $Y$ has the form:

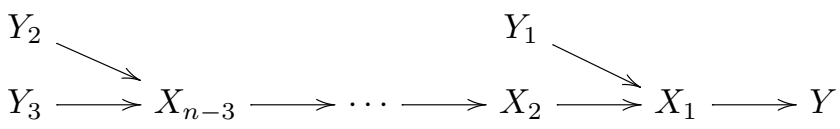

where $\partial\left(Y_{i}\right)=\partial(Y)=-1$ and $\partial\left(X_{i}\right)=-2$. The morphisms $Y_{i} \rightarrow Y$ are all sectional mono, but only the morphism $Y_{1} \rightarrow Y$ is good, since the other two factors through the branch modules $X_{n-3}$ and $X_{1}$.

Remark 54. We expect that there exists a more general notion of "good morphism" which holds for a larger class of quivers. It should be given by homological properties. The notion given here is adapted to the special choice of $Q$. The extra condition of not 
factoring through two branch modules only applies in type $\tilde{D}_{n}$ and it is needed to avoid pathological cases when $X$ is "close to the projectives".

We can now state the two fundamental lemmata to prove the main theorem of this section. Recall that a $Q$-representation $X$ is called thin if $\operatorname{dim} X_{i} \leq 1$ for every $i \in Q_{0}$. If the underlying graph of $Q$ is a tree then every indecomposable projective $Q$-representation is thin. If $Q$ is of type $\tilde{A}_{n}$ then all the indecomposable projective $Q$-representations are thin, except when $Q$ has precisely one source $i_{0}$ and precisely one sink $j_{0}$; in this case all projectives are thin except $P_{i_{0}}$ for which $\operatorname{dim}\left(P_{i_{0}}\right)_{j_{0}}=2$. Notice that $P_{i_{0}}$ is the only projective which is the end point of two non-isomorphic irreducible morphisms.

Lemma 55. Let $Y \in \mathcal{P}(Q)$ be indecomposable. Then, either $Y$ is a quotient of an indecomposable projective $Q$-representation which is thin or there exists a good mono $f: X \hookrightarrow Y$ ending in $Y$.

Proof. Let us consider the section $\Sigma$ ending in $Y$. If $\Sigma$ is complete, then a look at Table 1 reveals that there exists $X \in \Sigma$ such that $|\partial(X)| \leq|\partial(Y)|$ (since $Q$ is not of type $\tilde{E}_{8}$ ) and hence the morphism $f: X \rightarrow Y$ is a sectional mono (see Lemma 51). Thus we may choose $f$ to be a minimal sectional mono and in type $\tilde{D}_{n}$ such $X$ can be chosen so that the sectional morphism from $X$ to $Y$ does not factor through two branch modules. It follows that $f$ can be chosen to be a minimal good mono.

If $\Sigma$ is not complete, then it contains a projective $X$ and the sectional map $X \rightarrow Y$ is either mono or epi by Happel-Ringel Lemma 7. This concludes the proof.

Lemma 56. Let $f: X \hookrightarrow Y$ be a minimal good mono and let $S=\operatorname{Coker}(f)$. Then $[S, X]^{1}=1$ and hence $S^{X}$ and $X_{S}$ are well-defined. We have that $S^{X}=S$ and there are three possibilities for $X_{S}$ : either

(1) $X_{S}=0$ or

(2) $X_{S}$ is indecomposable and $X_{S} \hookrightarrow X$ is a good mono or

(3) $X_{S}=F \oplus T$ with $F$ and $T$ indecomposable, $F \hookrightarrow X$ irreducible and $T \hookrightarrow X / F$ good mono.

Moreover, $S$ is rigid, indecomposable and, if either preprojective or regular, it is quasisimple.

Proof. If $Q$ is the Kronecker quiver, then $f$ is irreducible and $S$ is regular quasi-simple of dimension vector $\delta=(1,1)$. In particular, $[S, X]^{1}=[X, S]=1$. Since $f$ is irreducible, $S^{X}=S$; moreover, by Lemma $31 X / X_{S} \cong S$ and $X_{S} \rightarrow X$ is irreducible.

Let us now suppose that $Q$ is affine, neither of type $\tilde{E}_{8}$ nor of Kronecker type. In this case the fact that $f$ is a minimal sectional mono implies that $[X, \tau Y]=0$ and $[X, Y]=1$. Thus, Unger's Lemma 8 guarantees that $S$ is indecomposable, rigid and 
$[S, X]^{1}=1$. It follows that $S^{X}$ and $X_{S}$ are well-defined (see Definition 28). Let us show that $S^{X}=S$. Let $\iota: N \hookrightarrow S$ be a proper non-zero subrepresentation of $S$. Since $f$ is a minimal sectional mono, the pullback sequence $\iota_{*}(\xi)$ splits. Since the morphism $\operatorname{Ext}^{1}(S, X) \rightarrow \operatorname{Ext}^{1}(N, X)$ is surjective and $[S, X]^{1}=1$, it follows that $[N, X]^{1}=0$. We conclude that $S^{X}=S$. Moreover, there are no irreducible monomorphisms ending in $S$. If $S$ is either preprojective or regular, Corollary 18 hence implies that $S$ is quasi-simple. Let us now compute $X_{S}$. Let $E \rightarrow X$ be the minimal right almost split map ending in $X$. Since $f: X \rightarrow Y$ is a sectional morphism, it has the form (16). Then $E=\tau X_{1} \oplus E^{\prime}$ for some $E^{\prime}$. By Lemma $36, X_{S} \cong \operatorname{ker}\left(\tau X_{1} \rightarrow \tau Y\right) \oplus E^{\prime}$. By a case by case direct inspection, we notice that the following remarkable properties of $\mathcal{P}(Q)$ hold:

The kernel of a sectional epi in $\mathcal{P}(Q)$ is a sectional mono;

The cokernel of an irreducible mono $L \hookrightarrow M$ in $\mathcal{P}(Q)$ with $|\partial(L)|<|\partial(M)|$

is a sectional epi.

(see Remark 57). We use (17) and (18) to show that $X_{S}$ has the claimed form.

Let us suppose first that $X$ is quasi-simple. Then $E^{\prime}=0$ and $X_{S}=\operatorname{ker}\left(\tau X_{1} \rightarrow \tau Y\right)$. By (17), if $X_{S} \neq 0$ then the embedding $X_{S} \hookrightarrow X$ is a sectional mono. If $Q$ is of type $\tilde{D}_{n}$ then $|\partial(X)|=1$ and $|\partial(Y)| \in\{1,2\}$; if $|\partial(Y)|=1$, then (since $f$ does not factor through two branch modules) $f=f_{2} \circ f_{1}$ where $f_{1}: X \rightarrow X_{1}$ and $f_{2}: X_{1} \rightarrow Y$ are irreducible, and $X_{1}$ is a branch module; in this case $X_{S} \rightarrow X$ has the form $X_{S} \rightarrow \tau X_{1} \rightarrow X$ where $X_{S} \rightarrow \tau X_{1}$ is irreducible and hence $X_{S} \rightarrow X$ is good mono. If $|\partial(Y)|=2$ then $|\partial(Y)|=\left|\partial\left(X_{1}\right)\right|=2$ and hence $X_{S}=0$.

Let us suppose that $X$ is a branch module. Then $E^{\prime}=E_{1} \oplus E_{2}$ where $E_{1}$ and $E_{2}$ are indecomposable. Since $f$ does not factor through two branch modules we have

$$
\operatorname{Ker}\left(\tau X_{1} \rightarrow \tau Y\right) \cong \operatorname{Ker}\left(X \rightarrow X_{t}\right) \subseteq \operatorname{Ker}(X \rightarrow Y)=0
$$

It follows that $X_{S}=E_{1} \oplus E_{2}$. By inspection, $|\partial(X)|>\left|\partial\left(E_{1}\right)\right|$; thus (18) guarantees that $X \rightarrow X / E_{1}$ is a sectional epi. Then $E_{2} \rightarrow X \rightarrow X / E_{1}$ is a sectional morphism.

Let us suppose that $X$ is neither quasi-simple nor a branch module. Then $E^{\prime}$ is either zero or indecomposable. If either $\operatorname{Ker}\left(\tau X_{1} \rightarrow \tau Y\right)=0$ or $E^{\prime}=0$, then $X_{S}$ has the claimed form. Let us suppose that they are both non-zero. We put $F:=E^{\prime}$ and $T:=\operatorname{Ker}\left(\tau X_{1} \rightarrow \tau Y\right)$. We claim that $T \rightarrow X / F$ is a sectional morphism. In view of (17), the morphism $T \rightarrow X$ is a sectional mono and it does not factor through $F$. To prove the claim it remains to check that $X \rightarrow X / F$ is a sectional morphism. By direct inspection, only two possibilities can occur: either $\left|\partial\left(X_{1}\right)\right|>|\partial(X)|>\left|\partial\left(E^{\prime}\right)\right|$ or $\left|\partial\left(E^{\prime}\right)\right|>|\partial(X)|>\left|\partial\left(X_{1}\right)\right|$. Since $X_{1}$ is closer to the branch module of the section ending in $Y$ than $X$, again by direct inspection, $\left|\partial\left(X_{1}\right)\right|>|\partial(X)|>|\partial(E)|$. By (18), the cokernel $X \rightarrow X / E^{\prime}$ is a sectional epi and we are done. 
Remark 57. The fact that the kernel of a sectional epi in $\mathcal{P}(Q)$ is a sectional mono is not true if $Q$ is of type $\tilde{E}_{8}$. This is the reason why $\tilde{E}_{8}$ needs a slightly different treatment.

Remark 58. If $f: X \rightarrow Y$ is a good mono, not necessarily minimal, then the description of $X_{S}$ given in Lemma 56 still holds. The minimality of the morphism is needed only to get $S^{X}=S$.

Theorem 59. Every preprojective and every preinjective representation of $Q$ has property $(C)$.

Proof. Up to duality, it is enough to show the claim for preprojective $Q$-representations. Since the category $\mathcal{P}(Q)$ of preprojective $Q$-representations is directed, in view of Theorem 26, it is enough to prove the claim for an indecomposable preprojective $Q$-representation.

Let $Y$ be an indecomposable preprojective $Q$-representation. If $Y$ is the quotient of an indecomposable projective $Q$-representation which is thin, then it is thin itself. Then every quiver Grassmannian attached to $Y$ is either empty or a point. If $Y$ is not a quotient of a projective indecomposable which is thin, then, by Lemma 55 there exists a good mono $f: X \hookrightarrow Y$ ending in $Y$. We use this monomorphism to define the open subset $\mathcal{U}_{\mathbf{e}}(Y, X):=\operatorname{Gr}_{\mathbf{e}}(Y) \backslash \operatorname{Gr}_{\mathbf{e}}(X)$ of $\operatorname{Gr}_{\mathbf{e}}(Y)$. There is the obvious decomposition $\operatorname{Gr}_{\mathbf{e}}(Y)=\operatorname{Gr}_{\mathbf{e}}(X) \coprod \mathcal{U}_{\mathbf{e}}(Y, X)$. By induction (on $\left.\operatorname{dim} Y\right) X$ has property (C) and hence $\operatorname{Gr}_{\mathbf{e}}(X)$ admits a cellular decomposition. Let us show that $\mathcal{U}_{\mathbf{e}}(Y, X)$ admits a cellular decomposition, too. We proceed by induction on the total dimension of $X$ and $Y$. If the sectional monomorphism $X \hookrightarrow Y$ is not minimal, then it splits as the composition of two sectional monomorphisms $X \hookrightarrow X^{\prime}$ and $X^{\prime} \hookrightarrow Y$ which are still good and such that $X^{\prime} \hookrightarrow Y$ is minimal. We have $\mathcal{U}_{\mathbf{e}}(Y, X)=\mathcal{U}_{\mathbf{e}}\left(Y, X^{\prime}\right) \amalg \mathcal{U}_{\mathbf{e}}\left(X^{\prime}, X\right)$ and hence by induction $\mathcal{U}_{\mathbf{e}}(Y, X)$ admits a cellular decomposition. We can hence assume that $f: X \hookrightarrow Y$ is minimal. Let $S$ be its cokernel. By Lemma $56, S^{X}=S$ and $X_{S}$ has one of the three forms shown there. Moreover, $S$ is rigid and indecomposable. If $S$ is regular, then it has property (C) by Proposition 48; if $S$ is not regular, then by induction we can assume that it has property (C) (if $S$ is preinjective, its dual is preprojective and property (C) is preserved by duality). By definition, $\mathcal{U}_{\mathbf{e}}(Y, X)=\coprod_{\mathbf{g} \neq 0} \mathcal{S}_{\mathbf{f}, \mathbf{g}}^{\xi}$, where $\xi \in \operatorname{Ext}^{1}(S, X)$ is the non-zero exact sequence induced by $f$. In view of Theorem 32, there are affine bundles

$$
\mathcal{S}_{\mathbf{f}, \mathbf{g}}^{\xi} \longrightarrow \operatorname{Gr}_{\mathbf{f}}(X) \times \operatorname{Gr}_{\mathbf{g}}(S) \text { for } \mathbf{g} \neq \operatorname{dim} S, \quad \mathcal{S}_{\mathbf{f}, \mathbf{g}}^{\xi} \longrightarrow \mathcal{U}_{\mathbf{f}}\left(X, X_{S}\right) \text { for } \mathbf{g}=\operatorname{dim} S
$$

By induction, $\operatorname{Gr}_{\mathbf{f}}(X) \times \operatorname{Gr}_{\mathbf{g}}(S)$ admits a cellular decomposition, and hence $\mathcal{S}_{\mathbf{f}, \mathbf{g}}^{\xi}$ admits a cellular decomposition, too, if $\mathbf{g} \neq \operatorname{dim} S$. It remains to show that $\mathcal{U}_{\mathbf{f}}\left(X, X_{S}\right)$ admits a cellular decomposition. We consider the three possibilities.

If $X_{S}=0$, then $\mathcal{U}_{\mathbf{f}}\left(X, X_{S}\right)=\operatorname{Gr}_{\mathbf{f}}(X)$ admits a cellular decomposition by induction.

If $X_{S}$ is of type (2), i.e. it is indecomposable and $X_{S} \hookrightarrow X$ is a good mono, then the inductive hypothesis guarantees that $\mathcal{U}_{\mathbf{f}}\left(X, X_{S}\right)$ admits a cellular decomposition. 
Suppose, next, that $X_{S}$ is of type (3), i.e. $X_{S}=F \oplus T \hookrightarrow X$ with $F \hookrightarrow X$ irreducible and $T \hookrightarrow X / F$ good mono. We have a commutative diagram with exact rows and columns:

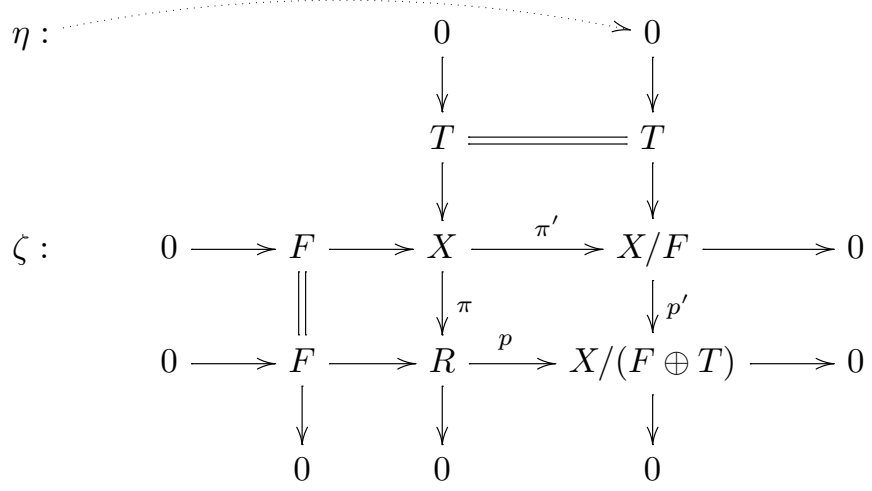

The middle row $\zeta$ and the righthand column $\eta$ satisfy the hypothesis of Theorem 32 . Moreover, since $F \rightarrow X$ is irreducible, $(X / F)^{F}=X / F$. We can hence decompose a quiver Grassmannian $\operatorname{Gr}_{\mathbf{e}}(X)$ as $\operatorname{Gr}_{\mathbf{e}}(X)=\amalg \mathcal{S}_{\mathbf{f}, \mathbf{g}}^{\zeta}$ and there are affine bundles

$$
\begin{aligned}
\mathcal{S}_{\mathbf{f}, \mathbf{g}}^{\zeta} \longrightarrow \operatorname{Gr}_{\mathbf{f}}(F) \times \operatorname{Gr}_{\mathbf{g}}(X / F) & \text { for } \mathbf{g} \neq \operatorname{dim}(X / F), \\
\mathcal{S}_{\mathbf{f}, \operatorname{dim} X / F}^{\zeta} \longrightarrow \mathcal{U}_{\mathbf{f}}\left(F, F_{X / F}\right) & \text { for } \mathbf{g}=\operatorname{dim}(X / F) .
\end{aligned}
$$

By definition, $\mathcal{U}_{\mathbf{e}}(X, F)=\coprod_{\mathbf{g} \neq 0} \mathcal{S}_{\mathbf{f}, \mathbf{g}}^{\zeta}$ and $\mathcal{U}_{\mathbf{e}}\left(X, X_{S}\right)=\coprod_{\mathbf{g} \neq 0} \mathcal{S}_{\mathbf{f}, \mathbf{g}}^{\zeta} \cap \mathcal{U}_{\mathbf{e}}\left(X, X_{S}\right)$. A point $N$ lies in $\mathcal{S}_{\mathbf{f}, \mathbf{g}}^{\zeta} \cap \mathcal{U}_{\mathbf{e}}\left(X, X_{S}\right)$ if and only if $p \circ \pi(N)=p^{\prime} \circ \pi^{\prime}(N) \neq 0$. This means that $N \in \mathcal{S}_{\mathbf{f}, \mathbf{g}}^{\zeta} \cap \mathcal{U}_{\mathbf{e}}\left(X, X_{S}\right)$ if and only if $\pi^{\prime}(N)$ belongs to $\mathcal{U}_{\mathbf{g}}(X / F, T)$. Thus, the affine bundles above restrict to affine bundles

$$
\begin{gathered}
\mathcal{S}_{\mathbf{f}, \mathbf{g}}^{\zeta} \cap \mathcal{U}_{\mathbf{e}}\left(X, X_{S}\right) \longrightarrow G r_{\mathbf{f}}(F) \times \mathcal{U}_{\mathbf{g}}(X / F, T) \quad \text { for } 0 \neq \mathbf{g} \neq \operatorname{dim}(X / F), \\
\mathcal{S}_{\mathbf{f}, \operatorname{dim} X / F}^{\zeta}=\mathcal{S}_{\mathbf{f}, \operatorname{dim} X / F}^{\zeta} \cap \mathcal{U}_{\mathbf{e}}\left(X, X_{S}\right) \longrightarrow \mathcal{U}_{\mathbf{f}}\left(F, F_{X / F}\right) \quad \text { for } \mathbf{g}=\operatorname{dim}(X / F) .
\end{gathered}
$$

By induction $\operatorname{Gr}_{\mathbf{f}}(F), \mathcal{U}_{\mathbf{g}}(X / F, T)$ and $\mathcal{U}_{\mathbf{f}}\left(F, F_{X / F}\right)$ admit a cellular decomposition, and so does $\mathcal{S}_{\mathbf{f}, \mathbf{g}}^{\zeta} \cap \mathcal{U}_{\mathbf{e}}\left(X, X_{S}\right)$ and hence $\mathcal{U}_{\mathbf{e}}\left(X, X_{S}\right)$.

\subsubsection{Type $\tilde{E}_{8}$}

Let $Q$ be an affine quiver of type $\tilde{E}_{8}$. In this section we prove:

Theorem 60. Every preprojective and every preinjective representation of $Q$ has property $(C)$. 
Up to duality, it is enough to show the claim for preprojective $Q$-representations. Since the category $\mathcal{P}(Q)$ of preprojective $Q$-representations is directed, in view of Theorem 26, it is enough to prove the claim for an indecomposable preprojective $Q$-representation.

We first prove the claim for representations of defect (-1) (see Proposition 64 below), then for the remaining quasi-simples (see Proposition 65 below) and finally for all the other indecomposable representations (see Proposition 67). Let $\delta$ be the minimal positive imaginary root of $q_{Q}$ (see Table 1). We denote by $e \in Q_{0}$ the extending vertex of $Q$, which is the unique vertex such that $\delta_{e}=1$. A representation of defect $(-1)$ is hence of the form $\tau^{-k} P_{e}$ for $k \geq 0$.

Lemma 61. For every $k \geq 0$ there exists a short exact sequence

$$
\xi_{k}: 0 \longrightarrow X:=\tau^{-k} P_{e} \longrightarrow Y:=\tau^{-6} X \longrightarrow S=S_{k} \longrightarrow 0
$$

such that

(1) $S$ is regular quasi-simple in the tube of rank 5 and $[S, X]^{1}=1$.

(2) If $k \geq 6, X_{S}=\tau^{6} X$ and $S^{X}=S$.

(3) If $k=5, \xi_{5}$ is generalized almost split (i.e. $X_{S}=0$ and $S^{X}=S$ ).

(4) If $k \leq 4, X_{S}=0$ and $S^{X}=\tau^{-} X$.

Proof. In Table 2 we reproduce the starting functions $s_{i}:=\operatorname{dim} \operatorname{Hom}\left(P_{i},-\right)\left(i \in Q_{0}\right)$ considered by Bongartz [4]. The starting function $s_{e}$ is in the last row. From this we see that $\left[P_{e}, \tau^{-6} P_{e}\right]=1$ and $\left[P_{e}, \tau^{-5} P_{e}\right]=0$; thus the same holds for the $\tau^{-}$-translates and we get $[X, Y]=1$ and $[X, \tau Y]=0$. Since $X$ and $Y$ have the same defect, it follows from Lemma 51 that $X$ embeds into $Y$ and the sequence $\xi_{k}$ exists for every $k \geq 0$. Moreover, by Unger's Lemma, we have that $[S, X]^{1}=1$ and that $S$ is rigid indecomposable. Since $X$ and $Y$ have the same defect, $S$ has defect zero and hence it is regular and must belong to an exceptional tube. Since, again by Table 2, there are no $Z$ such that $X \subset Z \subset Y$ and $\partial(Z)=(-1)$, it follows that $S$ is quasi-simple.

Suppose that $k \geq 6$, so that $\tau^{6} X$ is non-zero. Again from Bongartz's table (last row of Table 2) we see that $\left[P_{e}, \tau^{-12} P_{e}\right]=1$ and $\left[P_{e}, \tau^{-11} P_{e}\right]=0$; thus the same holds for the $\tau^{-}$-translates and we get $\left[\tau^{6} X, Y\right]=1$ and $\left[\tau^{6} X, \tau Y\right]=0$. Thus, $\tau^{6} X$ embeds both into $X$ and into $Y$ and we get an induced short exact sequence of quotients

$$
0 \longrightarrow X / \tau^{6} X \longrightarrow Y / \tau^{6} X \longrightarrow S \longrightarrow 0
$$

where $X / \tau^{6} X$ and $S$ are indecomposable regular quasi-simple. From Unger's lemma, we deduce that the middle term $Y / \tau^{6} X$ is indecomposable. This implies that the sequence (19) is almost split and hence $X / \tau^{6} X \cong \tau S$. It follows that $X_{S}=\tau^{6} X$. Moreover, since $X / X_{S}=\tau S$, it follows that $S^{X}=S$. Let us now show that $\tau^{5} S_{k} \cong S_{k}$ for any $k \geq 0$ and hence that $S_{k}$ belongs to the tube of rank 5 . Suppose that $k \geq 6$. Then $\tau^{6} X=X_{S} \neq 0$ and $\xi_{k-6}$ is given by 


\section{Table 2}

The starting functions $s_{P_{i}}:=\operatorname{dim} \operatorname{Hom}\left(P_{i},-\right)$ for $i \in Q_{0}$.

\begin{tabular}{|c|c|}
\hline 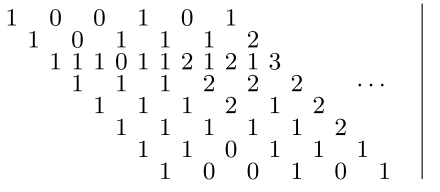 & 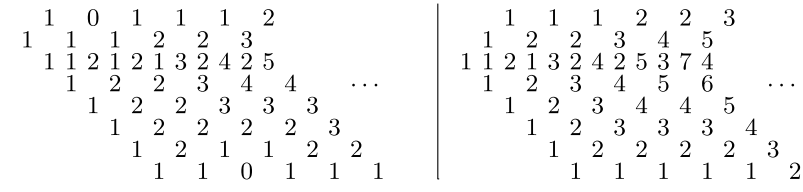 \\
\hline 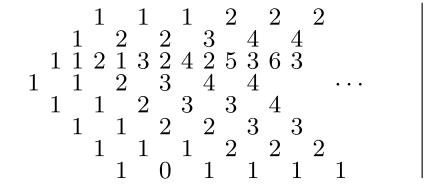 & 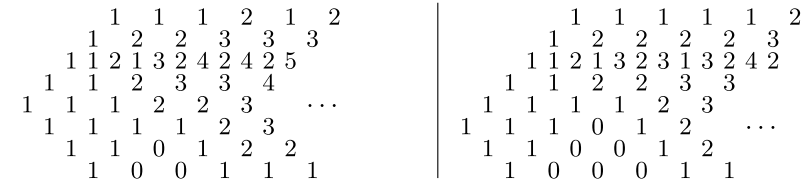 \\
\hline 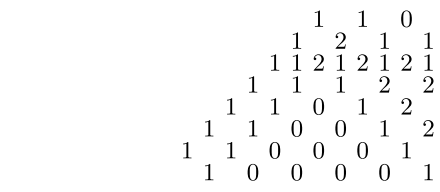 & 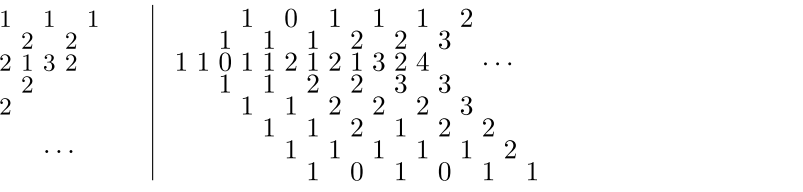 \\
\hline
\end{tabular}

$$
\begin{aligned}
& { }_{1} \begin{array}{llllllllllll}
1 & 0 & 0 & 0 & 1 & 0 & 1 & 1 & 0 & 0 & 1 & 1
\end{array}{ }^{1}{ }_{2} 1{ }_{1} 0
\end{aligned}
$$

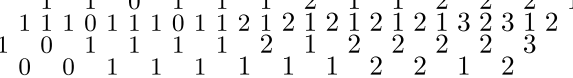

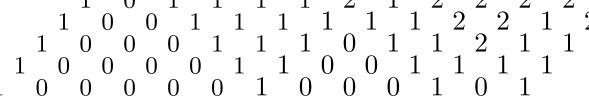

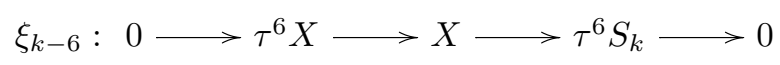

Since $X / \tau^{6} X=\tau S$, we see that $\tau^{6} S_{k} \cong \tau S_{k}$ and hence $\tau^{5} S \cong S$. Since the exceptional tubes of a quiver of type $\tilde{E}_{8}$ have rank 2,3 and 5 , the only possibility is that $S$ belongs to the tube of rank 5 .

Suppose now that $k \leq 5$ so that $\tau^{6} X=0$. Let us compute $X_{S}=\operatorname{ker}(X \rightarrow \tau S)$ and $S^{X}=\operatorname{im}\left(\tau^{-} X \rightarrow S\right)$. We apply the functor $\tau^{6}$ to $\xi_{k}$ and since $\tau^{6} X=0$ we get the short exact sequence

$$
0 \longrightarrow \tau^{6} Y=X \longrightarrow \tau^{6} S=\tau S \longrightarrow \tau^{5-k} I_{e} \longrightarrow 0
$$

(Here we have used the fact that $\nu\left(P_{e}\right)=I_{e}$.) From this we see that $X$ embeds into $\tau S$ and hence $X_{S}=0$. To compute $S^{X}$ we apply $\tau^{-}$to (20) and get the short exact sequence

$$
\xi_{k}^{\prime}: 0 \longrightarrow \tau^{-} X \longrightarrow S \longrightarrow \tau^{4-k} I_{e} \longrightarrow 0
$$

From this, we see that the map $\tau^{-} X \rightarrow S$ is injective for $k \leq 4$ and it is surjective for $k=5$. This concludes the proof. 
Lemma 62. For $0 \leq k \leq 4$ both $\tau^{-k} P_{e}$ and $\tau^{k} I_{e}$ are thin. A quiver Grassmannian associated with $\tau^{-5} P_{e}$ is either empty or a point or $\mathbf{P}^{1}$. In particular, $\tau^{-k} P_{e}$ has property (C), for every $k \in[0,5]$.

Proof. The fact that $\tau^{-k} P_{e}$ is thin for $k \in[0,4]$ follows from Table 2. By duality, the same holds for $\tau^{k} I_{e}$. If $X=\tau^{-5} P_{e}$, then again by Table 2, its dimension vector is estimated as

$$
\operatorname{dim}\left(\tau^{-5} P_{e}\right) \leq 01111211
$$

Suppose that $X$ is not thin (otherwise we are done) and hence $\operatorname{dim}(X)_{i}=2$ where $i$ is the branch vertex. Let us consider a non-empty quiver Grassmannian $\operatorname{Gr}_{\mathbf{e}}(X)$. If $\mathbf{e}_{i} \neq 1$ then $\operatorname{Gr}_{\mathbf{e}}(X)$ is either empty or a point because it can be identified with the quiver Grassmannian of a thin representation. Suppose $\mathbf{e}_{i}=1$. We have a natural map $\operatorname{Gr}_{\mathbf{e}}(X) \rightarrow \mathbf{P}^{1}$ which is projection to vertex $i$. This is an algebraic map of projective irreducible varieties (indeed $\operatorname{Gr}_{\mathbf{e}}(X)$ is irreducible in view of Proposition 38). So the image is closed and irreducible. It is hence either a point or $\mathbf{P}^{1}$. This map is injective because outside of the branch vertex $X$ is thin and hence there is at most one way to extend a point of $\mathbf{P}^{1}=\mathbf{P}\left(X_{i}\right)$ to a subrepresentation of $X$ of dimension vector $\mathbf{e}$.

Lemma 63. Let $X=\tau^{-k} P_{e}$ for $k \in[0,5]$. Let us consider the s.e.s.

$$
\xi_{k}: 0 \longrightarrow X \longrightarrow Y:=\tau^{-6} X \longrightarrow S \longrightarrow 0
$$

Then $Y$ has property $(C)$ and $\mathcal{U}_{\mathbf{e}}(Y, X)$ admits a cellular decomposition for every $\mathbf{e}$.

Proof. By Lemma 61, $\xi_{5}$ is a generalized almost split sequence and hence, since both $X$ and $S$ have property (C), then $Y$ has property $(\mathrm{C})$. Moreover, $\mathcal{U}_{\mathbf{e}}(Y, X)=\coprod_{\mathbf{g} \neq \mathbf{0}} \mathcal{S}_{\mathbf{f}, \mathbf{g}}^{\xi_{5}}$ and there are affine bundles $\mathcal{S}_{\mathbf{f}, \mathbf{g}}^{\xi_{5}} \longrightarrow \operatorname{Gr}_{\mathbf{f}}(X) \times \operatorname{Gr}_{\mathbf{g}}(S)$ from which we deduce that $\mathcal{U}_{\mathbf{e}}(Y, X)$ admits a cellular decomposition.

Let us now assume that $0 \leq k \leq 4$. By Lemma 61 we have $[S, X]^{1}=1, X_{S}=0$ and $S^{X}=\tau^{-} X$. Thus there are affine bundles

$$
\begin{gathered}
\mathcal{S}_{\mathbf{f}, \mathbf{g}}^{\xi_{k}} \longrightarrow \operatorname{Gr}_{\mathbf{f}}(X) \times \operatorname{Gr}_{\mathbf{g}}(S) \text { for } \mathbf{f} \neq \mathbf{0}, \\
\mathcal{S}_{\mathbf{0}, \mathbf{e}}^{\xi_{k}} \longrightarrow \operatorname{Gr}_{\mathbf{e}}(S) \backslash \operatorname{Gr}_{\mathbf{e}-\operatorname{dim} \tau^{-} X}\left(S / \tau^{-} X\right) \text { for } \mathbf{f}=\mathbf{0} .
\end{gathered}
$$

Since $X$ and $S$ have property $(\mathrm{C}), \mathcal{S}_{\mathbf{f}, \mathbf{g}}^{\xi_{k}}$ admits a cellular decomposition for $\mathbf{f} \neq \mathbf{0}$. Let us show that the same holds for $\mathcal{S}_{\mathbf{0}, \mathbf{e}}^{\xi_{k}}$. Let $\xi_{k}^{\prime}: 0 \rightarrow \tau^{-} X \rightarrow S \rightarrow \tau^{4-k} I_{e} \rightarrow 0$ be the short exact sequence (21). Then, by definition, $\operatorname{Gr}_{\mathbf{e}}(S) \backslash \operatorname{Gr}_{\mathbf{e}-\operatorname{dim} \tau^{-} X}\left(S / \tau^{-} X\right)=\coprod_{\mathbf{f} \neq \mathbf{0}} \mathcal{S}_{\mathbf{f}, \mathbf{g}}^{\xi_{k}^{\prime}}$. 
Since $\left[\tau^{4-k} I_{e}, \tau^{-} X\right]^{1}=\left[\tau^{4-k} I_{e}, \tau^{-k-1} P_{e}\right]^{1}=\left[\tau^{-k-1} P_{e}, \tau^{5-k} I_{e}\right]=\left[P_{e}, \tau^{6} I_{e}\right]=1$, we can use Theorem 32 to get algebraic maps which are affine bundles on their image

$$
\Psi_{\mathbf{f}, \mathbf{g}}^{\xi_{k}^{\prime}}: \mathcal{S}_{\mathbf{f}, \mathbf{g}}^{\xi_{k}^{\prime}} \longrightarrow \operatorname{Gr}_{\mathbf{f}}\left(\tau^{-} X\right) \times \operatorname{Gr}_{\mathbf{g}}\left(\tau^{4-k} I_{e}\right)
$$

If $0 \leq k \leq 3$, then both $\tau^{-} X$ and $\tau^{4-k} I_{e}$ are thin by Lemma 62 and hence their quiver Grassmannians are either empty or a point. It follows that the image of $\Psi_{\mathbf{f}, \mathbf{g}}^{\xi_{k}^{\prime}}$ is either empty or a point and hence $\mathcal{S}_{\mathbf{f}, \mathbf{g}}^{\xi_{k}^{\prime}}$ is either empty or an affine space, in this case. If $k=4$, then $\operatorname{Gr}_{\mathbf{f}}\left(\tau^{-} X\right)=\operatorname{Gr}_{\mathbf{f}}\left(\tau^{-5} P_{e}\right)$ is either empty or a point or $\mathbf{P}^{1}$ while $\operatorname{Gr}_{\mathbf{g}}\left(I_{e}\right)$ is either empty or a point. The image of $\Psi_{\mathbf{f}, \mathbf{g}}^{\xi_{4}^{\prime}}$ is hence obtained from $\operatorname{Gr}_{\mathbf{f}}\left(\tau^{-} X\right)$ by removing $\operatorname{Gr}_{\mathbf{f}}\left(\left(\tau^{-} X\right)_{I_{e}}\right)$. In view of Proposition 35 (part $\left.(2)\right),\left(\tau^{-} X\right)_{I_{e}}$ is rigid and hence, by Proposition 38, $\operatorname{Gr}_{\mathbf{f}}\left(\left(\tau^{-} X\right)_{I_{e}}\right)$ is irreducible. It follows that if $\operatorname{Gr}_{\mathbf{f}}\left(\tau^{-} X\right)$ is isomorphic to $\mathbf{P}^{1}, \operatorname{Gr}_{\mathbf{f}}\left(\left(\tau^{-} X\right)_{I_{e}}\right)$ is either empty or a point or $\mathbf{P}^{1}$. In all cases, we see that the image of $\Psi_{\mathbf{f}, \mathbf{g}}^{\xi_{4}^{\prime}}$ is an affine space, and hence $\mathcal{S}_{\mathbf{f}, \mathbf{g}}^{\xi_{4}^{\prime}}$ is an affine space, too.

Proposition 64. If $Y=\tau^{-k} P_{e} \in \mathcal{P}(Q)$ has defect $(-1)$, then $Y$ has property $(C)$ and, for $k \geq 6$, both $\mathcal{U}_{\mathbf{e}}\left(Y, \tau^{6} Y\right)$ and $\operatorname{Gr}_{\mathbf{e}}(Y) \backslash\left(\mathrm{Gr}_{\mathbf{e}-\operatorname{dim} \tau^{6} Y}\right)\left(Y / \tau^{6} Y\right)$ admit a cellular decomposition, for every $\mathbf{e}$.

Proof. We proceed by induction on $k \geq 0$. If $k \in[0,5]$ then $Y$ has property (C) by Lemma 62. If $6 \leq k \leq 11$ then, by Proposition $63, Y$ has property $(\mathrm{C})$ and $\mathcal{U}_{\mathbf{e}}\left(Y, \tau^{6} Y\right)$ admits a cellular decomposition. If $k \geq 12$, then there exists a short exact sequence $\xi=\xi_{k-6}: 0 \rightarrow \tau^{6} Y \rightarrow Y \rightarrow S \rightarrow 0$ with the good properties highlighted in Lemma 61 . There are affine bundles

$$
\begin{gathered}
\mathcal{S}_{\mathbf{f}, \mathbf{g}}^{\xi} \longrightarrow \operatorname{Gr}_{\mathbf{f}}\left(\tau^{6} Y\right) \times \operatorname{Gr}_{\mathbf{g}}(S) \text { for } \mathbf{g} \neq \operatorname{dim} S, \\
\mathcal{S}_{\mathbf{f}, \mathbf{g}}^{\xi} \longrightarrow \mathcal{U}_{\mathbf{f}}\left(\tau^{6} Y, \tau^{12} Y\right) \text { for } \mathbf{g}=\operatorname{dim} S .
\end{gathered}
$$

By induction and Proposition 48, both $\operatorname{Gr}_{\mathbf{f}}\left(\tau^{6} Y\right) \times \operatorname{Gr}_{\mathbf{g}}(S)$ and $\mathcal{U}_{\mathbf{f}}\left(\tau^{6} Y, \tau^{12} Y\right)$ admit a cellular decomposition and hence each stratum $\mathcal{S}_{\mathbf{f}, \mathbf{g}}^{\xi}$ admits a cellular decomposition, too. By definition, $\operatorname{Gr}_{\mathbf{e}}(Y) \backslash\left(\mathrm{Gr}_{\mathbf{e}-\operatorname{dim}} \tau^{6} Y\right)\left(Y / \tau^{6} Y\right)=\coprod_{\mathbf{f} \neq \mathbf{0}} \mathcal{S}_{\mathbf{f}, \mathbf{g}}^{\xi}$ and hence it admits a cellular decomposition.

Let us now deal with the remaining quasi-simple representations.

Proposition 65. Every quasi-simple preprojective $Q$-representation has property $(C)$.

Proof. By Proposition 64 the claim holds for the quasi-simple representations of defect $(-1)$. Let us now deal with the remaining ones, which have defect either $(-2)$ or $(-3)$.

Let $X \in \mathcal{P}(Q)$ be quasi-simple of defect $(-2)$ and let $Y \in \mathcal{P}(Q)$ be the quasi-simple of defect $(-1)$ such that $X$ lies in the section ending in $Y$ (see Fig. 1). By direct check, 


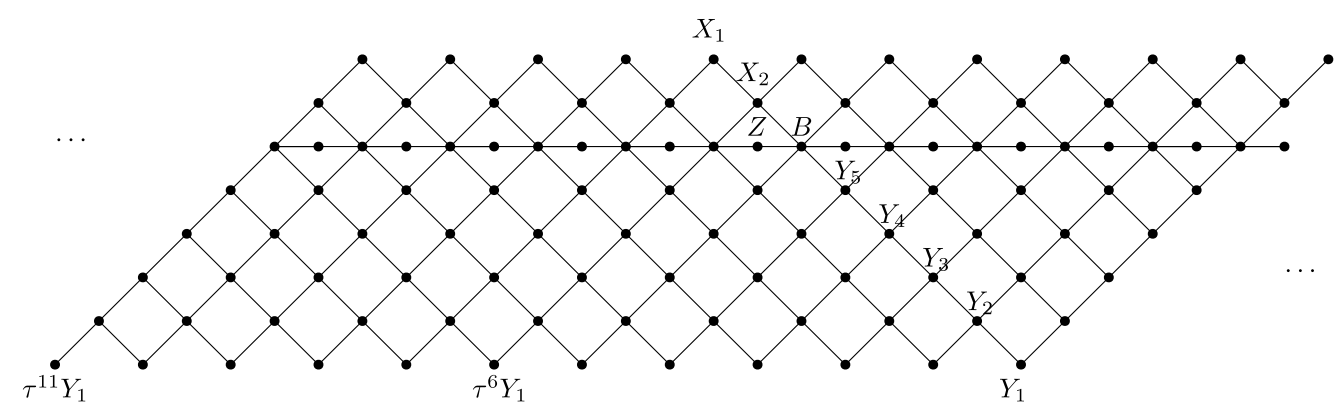

Fig. 1. Preprojective component of the AR-quiver of a quiver of type $\tilde{E}_{8}$.

one verifies that the kernel of the sectional morphism $X \rightarrow Y$ is the sectional morphism $\tau^{7} Y \rightarrow X$. If $\tau^{7} Y=0$ then $\tau^{6} Y$ is either projective or zero; in both cases $\left[P_{e}, X\right]=0$ and hence $X$ is supported on a Dynkin quiver of type $E_{8}$. Thus, by Theorem $46, X$ has property (C). Let us hence suppose that $\tau^{7} Y \neq 0$. Then we consider the non-split short exact sequence

$$
\xi: 0 \longrightarrow \tau^{7} Y \longrightarrow X \longrightarrow Y \longrightarrow 0 .
$$

Since $\left[Y, \tau^{7} Y\right]^{1}=\left[\tau^{7} Y, \tau Y\right]=\left[\tau^{6} Y, Y\right]=1$, we see that $\xi$ is generating. In order to get $\left(\tau^{7} Y\right)_{Y}$ and $Y^{\left(\tau^{7} Y\right)}$ (see Definition 28) the following lemma is needed.

Lemma 66. Let $Y_{1}$ and $Y_{2}$ be two indecomposable preprojective representations of defect $(-1)$ and let $f: Y_{1} \rightarrow Y_{2}$ be a non-zero homomorphism between them. Then $\operatorname{ker}(f)$ is zero and $f$ is injective.

Proof. From the exact sequence $0 \rightarrow \operatorname{ker}(f) \rightarrow Y_{1} \rightarrow \operatorname{im}(f) \rightarrow 0$ we have $-1=\partial\left(Y_{1}\right)=$ $\partial(\operatorname{ker}(f))+\partial(\operatorname{im}(f))$. Since $\operatorname{im}(f)$ is a non-zero sub-representation of $Y_{2}$, it is preprojective and hence it has negative defect. Then $\operatorname{ker}(f)$ must be zero, because otherwise it has negative defect as well, being a sub-representation of $Y_{1}$.

By Lemma 66 we have $\left(\tau^{7} Y\right)_{Y}=\operatorname{Ker}\left(\tau^{7} Y \rightarrow \tau Y\right)=0$, and $(Y)^{\tau^{7} Y}=\operatorname{Im}\left(\tau^{6} Y \rightarrow\right.$ $Y)=\tau^{6} Y$. By Theorem 32, there are (Zariski-locally trivial) affine bundles

$$
\begin{gathered}
\mathcal{S}_{\mathbf{f}, \mathbf{g}}^{\xi} \longrightarrow \operatorname{Gr}_{\mathbf{f}}\left(\tau^{7} Y\right) \times \operatorname{Gr}_{\mathbf{g}}(Y) \text { for } \mathbf{f} \neq \mathbf{0}, \\
\mathcal{S}_{\mathbf{0}, \mathbf{e}}^{\xi} \longrightarrow \operatorname{Gr}_{\mathbf{e}}(Y) \backslash \operatorname{Gr}_{\mathbf{e}-\operatorname{dim} \tau^{6} Y}\left(Y / \tau^{6} Y\right) \text { for } \mathbf{f}=\mathbf{0} .
\end{gathered}
$$

By Proposition 64, both $\operatorname{Gr}_{\mathbf{f}}\left(\tau^{7} Y\right) \times \operatorname{Gr}_{\mathbf{g}}(Y)$ and $\operatorname{Gr}_{\mathbf{e}}(Y) \backslash \operatorname{Gr}_{\mathbf{e}-\operatorname{dim} \tau^{6} Y}\left(Y / \tau^{6} Y\right)$ admit a cellular decomposition and hence each stratum $\mathcal{S}_{\mathbf{f}, \mathbf{g}}^{\xi}$ has the same property, proving property $(\mathrm{C})$ for $X$.

Let $Z \in \mathcal{P}(Q)$ be quasi-simple of defect $(-3)$ and let $X^{\prime} \in \mathcal{P}(Q)$ be the quasi-simple of defect $(-2)$ such that $Z$ lies in the section ending in $X^{\prime}$ (see Fig. 1). By direct check, 
one verifies that the kernel of the sectional morphism $Z \rightarrow X^{\prime}$ is the sectional morphism $Y^{\prime} \rightarrow Z$, where $Y^{\prime}$ is the quasi-simple of defect $(-1)$ in the section ending in $Z$. If $Y^{\prime}=0$ then $\left[P_{e}, Z\right]=0$ and hence $Z$ is supported on a quiver of type $E_{8}$ and by Theorem $46, Z$ has property $(\mathrm{C})$. Let us hence suppose that $Y^{\prime} \neq 0$. Then there is a short exact sequence

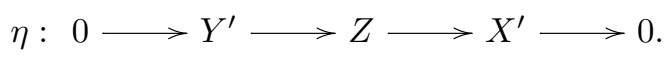

We have $\left[X^{\prime}, Y^{\prime}\right]^{1}=\left[Y^{\prime}, \tau X^{\prime}\right]=1$ and hence $\left(Y^{\prime}\right)_{X^{\prime}}$ and $X^{\prime}\left(Y^{\prime}\right)$ are well-defined (see Definition 28). For defect reasons, the sectional morphisms $Y^{\prime} \rightarrow \tau X^{\prime}$ and $\tau^{-} Y^{\prime} \rightarrow X^{\prime}$ are monomorphisms, and hence $\left(Y^{\prime}\right)_{X^{\prime}}=0$ and $X^{\prime\left(Y^{\prime}\right)}=\tau^{-} Y^{\prime}$. It follows that there are (Zariski-locally trivial) affine bundles

$$
\begin{gathered}
\mathcal{S}_{\mathbf{f}, \mathbf{g}}^{\eta} \longrightarrow \operatorname{Gr}_{\mathbf{f}}\left(Y^{\prime}\right) \times \operatorname{Gr}_{\mathbf{g}}\left(X^{\prime}\right) \text { for } \mathbf{f} \neq \mathbf{0}, \\
\mathcal{S}_{\mathbf{0}, \mathbf{e}}^{\eta} \longrightarrow \operatorname{Gr}_{\mathbf{e}}\left(X^{\prime}\right) \backslash \operatorname{Gr}_{\mathbf{e}-\operatorname{dim} \tau^{-} Y^{\prime}}\left(X^{\prime} / \tau^{-} Y^{\prime}\right) \text { for } \mathbf{f}=\mathbf{0} .
\end{gathered}
$$

We have already proved that $\operatorname{Gr}_{\mathbf{f}}\left(Y^{\prime}\right) \times \operatorname{Gr}_{\mathbf{g}}\left(X^{\prime}\right)$ admits a cellular decomposition. To get a cellular decomposition of $\operatorname{Gr}_{\mathbf{e}}\left(X^{\prime}\right) \backslash \mathrm{Gr}_{\mathbf{e}-\operatorname{dim} \tau^{-} Y^{\prime}}\left(X^{\prime} / \tau^{-} Y^{\prime}\right)$ we consider the short exact sequence

$$
\xi^{\prime}: 0 \longrightarrow \tau^{-} Y^{\prime} \longrightarrow X^{\prime} \longrightarrow \tau^{-8} Y^{\prime} \longrightarrow 0 \text {. }
$$

We proved above that each stratum $\mathcal{S}_{\mathbf{f}, \mathbf{g}}^{\xi^{\prime}}$ admits a cellular decomposition. It follows that $\operatorname{Gr}_{\mathbf{e}}\left(X^{\prime}\right) \backslash \operatorname{Gr}_{\mathbf{e}-\operatorname{dim} \tau^{-} Y^{\prime}}\left(X^{\prime} / \tau^{-} Y^{\prime}\right)=\coprod_{\mathbf{f} \neq 0} \mathcal{S}_{\mathbf{f}, \mathbf{g}}^{\xi^{\prime}}$ admits a cellular decomposition, too. This concludes the proof.

Proposition 67. An indecomposable preprojective representation of $Q$ has property (C).

Proof. Let $Y$ be an indecomposable preprojective representation of $Q$. If $Y$ is projective, then it is thin and hence it has property (C). If $Y$ is quasi-simple, then $Y$ has property (C) by Proposition 65. Suppose that $Y$ is not projective and not quasi-simple. Then there is an irreducible monomorphism $\iota: X \hookrightarrow Y$ ending in $Y$ and starting in a module $X$ such that $|\partial(X)|<|\partial(Y)|$ (see Table 1 ). Let $S=\operatorname{Coker}(\iota)$. Since $\iota$ is irreducible, $S$ is quasi-simple and preprojective. Moreover $[X, Y]=1$ and hence, by Unger's Lemma 8, $[S, X]^{1}=1$. The short exact sequence

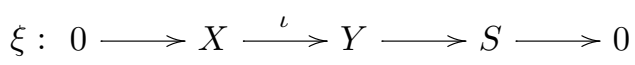

is thus generating and we can apply Theorem 32. By Proposition 65, $S$ has property (C). If $X$ is quasi-simple then $\xi$ is almost split and hence $Y$ has property (C) (see Corollary 33). If $X$ is not quasi-simple, it is not a branch module, for defect reasons, and 
hence, by Lemma $36, X_{S}$ is indecomposable and the embedding $X_{S} \rightarrow X$ is irreducible. There are affine bundles

$$
\mathcal{S}_{\mathbf{f}, \mathbf{g}}^{\xi} \longrightarrow \operatorname{Gr}_{\mathbf{f}}(X) \times \operatorname{Gr}_{\mathbf{g}}(S) \text { for } \mathbf{g} \neq \operatorname{dim} S, \quad \mathcal{S}_{\mathbf{f}, \mathbf{e}}^{\xi} \longrightarrow \mathcal{U}_{\mathbf{e}}\left(X, X_{S}\right) \text { for } \mathbf{g}=\operatorname{dim} S
$$

By induction, both $\operatorname{Gr}_{\mathbf{f}}(X) \times \operatorname{Gr}_{\mathbf{g}}(S)$ and $\mathcal{U}_{\mathbf{e}}\left(X, X_{S}\right)$ admit a cellular decomposition. We conclude that each stratum $\mathcal{S}_{\mathbf{f}, \mathbf{g}}^{\xi}$ admits a cellular decomposition, and hence $Y$ has property $(\mathrm{C})$.

\section{The cluster multiplication formula}

We recall the definition of the cluster character with coefficients $([22,28,30])$. Let $M$ be a finite dimensional representation of an acyclic quiver $Q$ with $n$ vertices. The $\mathbf{g}$-vector [22] or index [42] of $M$ is the integer vector $\mathbf{g}_{M} \in \mathbb{Z}^{Q_{0}}$ given by $\left(\mathbf{g}_{M}\right)_{i}:=-\left\langle S_{i}, M\right\rangle$. Let $H=\left(h_{i j}\right)_{i, j \in Q_{0}}$ be the matrix representing the Euler form $\langle-,-\rangle$ in the basis of simples: $\langle M, N\rangle={ }^{t}(\operatorname{dim} M) H(\operatorname{dim} N)$. The exchange matrix of $Q$ is defined as $B:=$ $H-H^{t}$. The CC-map is a map $M \mapsto C C(M)$ that associates to a representation of $Q$ a Laurent polynomial $C C(M) \in \mathbb{Z}\left[y_{1}, \cdots, y_{n}, x_{1}^{ \pm 1}, \cdots, x_{n}^{ \pm 1}\right]$. Given an integer vector $\mathbf{b}=$ $\left(b_{1}, \cdots, b_{n}\right)$ we use the shorthand notation $\mathbf{x}^{\mathbf{b}}:=x_{1}^{b_{1}} x_{2}^{b_{2}} \cdots x_{n}^{b_{n}}$. The Laurent polynomial $C C(M)$ is defined as follows

$$
C C(M):=\sum_{\mathbf{e}} \chi\left(\operatorname{Gr}_{\mathbf{e}}(M)\right) \mathbf{y}^{\mathbf{e}} \mathbf{x}^{B \mathbf{e}+\mathbf{g}_{M}}
$$

Given two $Q$-representations $X$ and $S$ such that $[S, X]^{1}=1$, a non-zero vector $\xi^{\prime} \in$ $\operatorname{Ext}^{1}\left(S^{X}, X / X_{S}\right)$ is a generalized almost split sequence and there exists an exact sequence $0 \rightarrow X / X_{S} \rightarrow \tau S^{X} \rightarrow I \rightarrow 0$ where $I$ is either injective or zero (see Lemma 31). We consider the indecomposable decomposition $I=I_{1}^{f_{1}} \oplus I_{2}^{f_{2}} \oplus \cdots \oplus I_{n}^{f_{n}}$ of $I$. Let $\mathbf{f}=\left(f_{1}, \cdots, f_{n}\right)$. We can now state and prove a multiplication formula between cluster characters with coefficients.

Theorem 68. Let $\xi \in \operatorname{Ext}^{1}(S, X)$ be a generating extension with middle term $Y$. Then

$$
C C(X) C C(S)=C C(Y)+\mathbf{y}^{\operatorname{dim} S^{X}} C C\left(X_{S} \oplus S / S^{X}\right) \mathbf{x}^{\mathbf{f}}
$$

If, in addition, $\operatorname{Ext}^{1}(X, S)=0$ and both $X$ and $S$ are exceptional then formula (23) is an exchange relation between the cluster variables $C C(X)$ and $C C(S)$ for the cluster algebra $\mathcal{A}_{\bullet}(\Sigma)$ with principal coefficients at the initial seed $\Sigma=(Q, \mathbf{x})$.

Proof. By using Corollary 34, we easily get the following formula:

$$
C C(X) C C(S)=C C(Y)+\mathbf{y}^{\operatorname{dim} S^{X}} \mathbf{x}^{\left.B \operatorname{dim} S^{X}+\mathbf{g}_{\left(X / X_{S}\right)}+\mathbf{g}_{(S} X\right)} C C\left(X_{S} \oplus S / S^{X}\right)
$$


Since $\mathbf{x}^{-\mathbf{g}_{I_{k}}}=x_{k}$ by definition of $\mathbf{g}$-vector, to prove formula (23) it remains to check that

$$
B \operatorname{dim} S^{X}+\mathbf{g}_{X / X_{S}}+\mathbf{g}_{S^{X}}=-\mathbf{g}_{I}
$$

To prove this we recall some properties of $\mathbf{g}$-vectors. Given a $Q$-representation $M$, by definition we have $\mathbf{g}_{M}=-H \operatorname{dim} M$. We define the coindex of $M$ as $\mathbf{g}^{M}:=-H^{t} \mathbf{d i m} M$, where $H^{t}$ denotes the transpose matrix. If $0 \rightarrow A \rightarrow B \rightarrow C \rightarrow 0$ is a short exact sequence, then $\mathbf{g}_{A \oplus C}=\mathbf{g}_{A}+\mathbf{g}_{C}=\mathbf{g}_{B}$. If $M$ is indecomposable non-projective, the following formula holds (see e.g. [9, Sec. 5.1]) $\mathbf{g}^{M}=-\mathbf{g}_{\tau M}$. Now (24) follows at once from these properties and concludes the proof of (23).

If $X$ and $S$ are exceptional, and $\operatorname{Ext}^{1}(X, S)=0$, then the two modules $Y$ and $X_{S} \oplus$ $S / S^{X}$ are rigid (see Proposition 35) and hence $C C(Y)$ and $C C\left(X_{S} \oplus S / S^{X}\right)$ are cluster monomials. Moreover $X \oplus Y \oplus X_{S} \oplus S / S^{X}$ and $S \oplus Y \oplus X_{S} \oplus S / S^{X}$ are rigid, too. To conclude we need to check that $[X, I]=[S, I]=[Y, I]=\left[X_{S}, I\right]=\left[S / S^{X}, I\right]=0$. This is done easily and concludes the proof.

Remark 69. In case $[X, S]^{1}=0$ and both $X$ and $S$ are exceptional, formula (23) makes clear that the c-vector of this exchange relation is the dimension vector of $S^{X}$. In particular, it is a positive real Schur root, since $S^{X}$ is a rigid brick (see Proposition 35). This provides a representation theoretic interpretation of those $\mathbf{c}$-vectors. The $\mathbf{c}-$ vectors of cluster algebras have been studied by several authors: [41] (for finite type cluster algebras), [54] and [13] (for general skew-symmetric cluster algebras). More recently, c--vectors have appeared in $\tau$-tilting theory $[21,1]$.

Example 70. Let $M$ be a rigid indecomposable non-projective representation of an acyclic quiver. Let $\xi: 0 \rightarrow \tau M \rightarrow E \rightarrow M \rightarrow 0$ be the almost split sequence ending in $M$. Then

$$
C C(M) C C(\tau M)=C C(E)+\mathbf{y}^{\operatorname{dim} M}
$$

Indeed, since $M$ is exceptional, $[M, \tau M]^{1}=[\tau M, \tau M]=[M, M]=1$. The generating extension $\xi \in \operatorname{Ext}^{1}(M, \tau M)$ is an almost split sequence. It follows that $M^{\tau M}=M$ and $(\tau M)_{M}=M$ (see Definition 28). Thus formula (25) is a particular case of the cluster multiplication formula (23). For almost split sequences, formula (25) holds in full generality [24].

\section{Acknowledgments}

We thank Antonio Rapagnetta, Corrado De Concini, Claus Michael Ringel, Bernhard Keller, Julia Sauter and Ernesto Mistretta for helpful discussions and comments.

\section{References}

[1] S. Asai, Semibricks, Int. Math. Res. Not. 2020 (16) (2020) 4993-5054. 
[2] I. Assem, D. Simson, A. Skowroński, Elements of the Representation Theory of Associative Algebras. Vol. 1. Techniques of Representation Theory, London Mathematical Society Student Texts, vol. 65, Cambridge University Press, Cambridge, 2006.

[3] M. Auslander, I. Reiten, S.O. Smalø, Representation Theory of Artin Algebras, Cambridge Studies in Advanced Mathematics, vol. 36, Cambridge University Press, Cambridge, 1997, corrected reprint of the 1995 original.

[4] K. Bongartz, Critical simply connected algebras, Manuscr. Math. 46 (1984) 117-136.

[5] P. Caldero, F. Chapoton, Cluster algebras as Hall algebras of quiver representations, Comment. Math. Helv. 81 (2006) 595-616.

[6] P. Caldero, B. Keller, From triangulated categories to cluster algebras II, Ann. Sci. Éc. Norm. Supér. 39 (4) (2006) 983-1009.

[7] P. Caldero, B. Keller, From triangulated categories to cluster algebras, Invent. Math. 172 (2008) 169-211.

[8] G. Cerulli Irelli, Quiver Grassmannians associated with string modules, J. Algebraic Comb. 33 (2011) 259-276.

[9] G. Cerulli Irelli, Geometry of quiver Grassmannians of Dynkin type with applications to cluster algebras, in: Proceedings of EMS, Representation Theory- Current Trends and Perspectives, 2017.

[10] G. Cerulli Irelli, F. Esposito, Geometry of quiver Grassmannians of Kronecker type and applications to cluster algebras, Algebra Number Theory 5 (6) (2011).

[11] G. Cerulli Irelli, X. Fang, E. Feigin, G. Fourier, M. Reineke, Linear degeneration of flag varieties, Math. Z. 287 (1-2) (2017) 615-654.

[12] G. Cerulli Irelli, E. Feigin, M. Reineke, Quiver Grassmannians and degenerate flag varieties, Algebra Number Theory 6 (1) (2012).

[13] A.N. Chavez, On the c-vectors of an acyclic cluster algebra, Int. Math. Res. Not. 6 (2015) 1590-1600.

[14] W. Crawley-Boevey, Lectures on representations of quivers, available at the author's webpage.

[15] W. Crawley-Boevey, More lectures on representations of quivers, available at the author's webpage.

[16] W. Crawley-Boevey, Rigid integral representations of quivers, Conf. Proc. 18 (1996).

[17] C. De Concini, G. Lusztig, C. Procesi, Homology of the zero-set of nilpotent vector field on a flag manifold, J. Am. Math. Soc. 1 (1) (1988) 15-34.

[18] P. Deligne, La conjecture de Weil. I, Inst. Hautes Études Sci. Publ. Math. 43 (1974) $273-307$.

[19] P. Deligne, SGA 4 $\frac{1}{2}$, Lecture Notes in Mathematics, vol. 569, Springer-Verlag, Berlin, 1977.

[20] M. Demazure, P. Gabriel, Groupes algébriques. Tome I: Géométrie algébrique, généralités, groupes commutatifs. Avec un appendice it Corps de classes local par Michiel Hazewinkel, Masson \& Cie, Éditeur, Paris, 1970.

[21] L. Demonet, O. Iyama, G. Jasso, $\tau$-tilting, bricks and g-vectors, Int. Math. Res. Not. 2019 (3) (2019) $852-892$.

[22] H. Derksen, J. Weyman, A. Zelevinsky, Quivers with potentials and their representations II: applications to cluster algebras, J. Am. Math. Soc. 23 (3) (2010) 749-790.

[23] V. Dlab, C.M. Ringel, Indecomposable Representations of Graphs and Algebras, Memoirs of the AMS, 1976.

[24] S. Dominguez, C. Geiss, A Caldero-Chapoton formula for generalized cluster categories, J. Algebra 399 (2014) 887-893.

[25] G. Ellingsrud, S.A. Strømme, Towards the Chow ring of the Hilbert scheme of $\mathbf{P}^{2}$, J. Reine Angew. Math. 441 (1993) 33-44.

[26] S. Fomin, A. Zelevinsky, Cluster algebras. I. Foundations, J. Am. Math. Soc. 15 (2) (2002) $497-529$.

[27] H. Franzen, T. Weist, The value of the Kac polynomial at one, Q. J. Math. 69 (1) (2018) 13-32.

[28] C. Fu, B. Keller, On cluster algebras with coefficients and 2-Calabi-Yau categories, Trans. Am. Math. Soc. 362 (2) (2010) 859-895.

[29] W. Fulton, Intersection Theory, second edition, Ergebnisse der Mathematik und ihrer Grenzgebiete. 3. Folge. A Series of Modern Surveys in Mathematics, vol. 2, Springer-Verlag, Berlin, 1998.

[30] C. Geiss, B. Leclerc, J. Schröer, Semicanonical bases and preprojective algebras. II. A multiplication formula, Compos. Math. 143 (5) (2007) 1313-1334.

[31] A. Grothendieck, Éléments de géométrie algébrique. IV. Étude locale des schémas et des morphismes de schémas. III, Inst. Hautes Études Sci. Publ. Math. 28 (1966) 255.

[32] D. Happel, C.M. Ringel, Tilted algebras, Trans. Am. Math. Soc. 274 (2) (1982).

[33] A. Hubery, Acyclic cluster algebras via Ringel-Hall algebras, available at the author's webpage.

[34] A. Lascoux, Classes de Chern d'un produit tensoriel, C. R. Acad. Sci. Paris, Sér. A-B 286 (8) (1978) A385-A387. 
[35] O. Lorscheid, Schubert decompositions for quiver Grassmannians of tree modules, Algebra Number Theory 9 (6) (2015) 1337-1362.

[36] O. Lorscheid, T. Weist, Quiver Grassmannians of extended Dynkin type D - part 2: Schubert decompositions and F-polynomials, arXiv:1507.00392, 2015.

[37] O. Lorscheid, T. Weist, Quiver grassmannians of type extended Dynkin type D - part 1: Schubert systems and decompositions into affine spaces, Mem. Am. Math. Soc. 261 (1258) (2019).

[38] G. Lusztig, Comments on my papers, arXiv:1707.09368.

[39] D. Mumford, The Red Book of Varieties and Schemes, second, expanded edition, Springer-Verlag, Berlin, 1999.

[40] H. Nakajima, Quiver varieties and cluster algebras, Kyoto J. Math. 51 (1) (2011) 71-126.

[41] T. Nakanishi, S. Stella, Diagrammatic description of c-vectors and d-vectors of cluster algebras of finite type, Electron. J. Comb. 21 (1) (2014).

[42] Y. Palu, Cluster characters for 2-Calabi-Yau triangulated categories, Ann. Inst. Fourier (Grenoble) 58 (6) (2008) 2221-2248.

[43] Y. Palu, Cluster characters II: a multiplication formula, Proc. Lond. Math. Soc. (3) 104 (1) (2012) $57-78$.

[44] F. Qin, Quantum cluster variables via Serre polynomials, J. Reine Angew. Math. 668 (2012) 149-190.

[45] D. Quillen, Projective modules over polynomial rings, Invent. Math. 36 (1976) 167-171.

[46] C.M. Ringel, Finite dimensional hereditary algebras of wild representation type, Math. Z. 161 (1978) $235-255$.

[47] C.M. Ringel, Reflection functors for hereditary algebras, J. Lond. Math. Soc. 21 (2) (1980) 465-479.

[48] C.M. Ringel, Tame Algebras and Integral Quadratic Forms, Lecture Notes in Mathematics, vol. 1099, Springer-Verlag, Berlin, 1984.

[49] C.M. Ringel, Exceptional modules are tree modules, Linear Algebra Appl. 275/276 (1998) 471-493.

[50] C.M. Ringel, Quiver Grassmannians for wild acyclic quivers, Proc. Am. Math. Soc. 146 (5) (2018).

[51] D. Rupel, T. Weist, Cell decomposition of rank two quiver Grassmannians, Math. Z. 295 (3-4) (2020) 993-1038.

[52] A. Schofield, General representations of quivers, Proc. Lond. Math. Soc. 65 (3) (1992) 46-64.

[53] D. Simson, A. Skowroński, Elements of the Representation Theory of Associative Algebras, vol. 2, Cambridge University Press, Cambridge, 2007.

[54] D. Speyer, H. Thomas, Acyclic cluster algebras revisited, in: H. Springer (Ed.), Algebras, Quivers and Representation, in: Abel Symp., vol. 8, 2013, pp. 275-298.

[55] C. Voisin, Chow Rings, Decomposition of the Diagonal, and the Topology of Families, vol. 187, Princeton University Press, Princeton, NJ, 2014.

[56] F. Xu, On the cluster multiplication theorem for acyclic cluster algebras, Trans. Am. Math. Soc. 362 (2) (2010) 753-776. 